\title{
Sanskritische Etymologien.
}

1. Skr. jihvä, av. hizvä u. s. w.

Es ist ganz natürlich, dass man die verschiedenen idg. Wörter für Zunge hat vereinigen wollen, nämlich l. dingua, lingua, g. tuggō, s. jihvá, zd. hizvā u. s. w. So z. B. Benfey Ergbl. z. allgem. Litteraturz. $1837908 \mathrm{f}$. $=\mathrm{Kl}$. Schr. II $7 \mathrm{f}$. L. Meyer Or. u. Occ. I 620, Got. Spr. 80, Grassmann Wb. z: Rgv., KZ. XI 12, Fick Wb. I 104 f. Dagegen stellen Pott I 230, ${ }^{2}$ II 2, 569 f., KZ. XXVI 153 und Roth im PW. sanskr. jih $v a ̈, ~ j u h \hat{u}-$ zur Wz. $h v a \bar{a}$-, $h \bar{u}$ - 'rufen' (vgl. Curtius Et. ${ }^{5}$ 194; Vaničk 340, fürs Lat. Corssen I 81, 223; II 274). Entschiedener hahen Lottner (KZ. VII 185), Schade (Wb. 1301 f.) und Bartholomae (KZ. XXVII 207 ff.) die arischen Wörter von den europäischen getrennt. Vom jetzigen Standpunkt der Frage muss man Bartholomae beipflichten. Anderseits aber sprechen sowohl die identische Bedeutung wie die teilweise formelle Übereinstimmung - vor allem in bezug auf Ableitung und Flexion (vgl. Möller P.-BrB. VII 544 ff.) - fur ursprtingliche Identität. Dieser Umstand mag den folgenden Erklärungsversuch motivieren.

Zunächst eine kurze Übersicht über die Formen der idg. Sprachen. Im S. begegnen jihvá und juhit-, im Av. hizav(N. hizu Gen. hizvō, Justi 324 f., Bartholomae Hdb. \& 230, vgl. BB. VIII 210, XIII 89 f.) und hizvä, hizu (Bartholomae Ar. F. II 142), ap. hizav-(parsi hizvann) ${ }^{1}$ ), wozu oss. äwzag (Hubschmann Etym. u. Lautl. d. oss. Spr. $\left.18 ; 80 ; 104 ; 108 ; 112)^{2}\right)$.

1) Über die ap. Lesung von diesem Wort Bh. II 74 s. Spiegel Ap. Keilinschr. 97; 211; Bartholomae Hdb. S. 6 n. 1; a. a. 0 . 208 n. 3; F. Müller Wien. Zs. I 223.

2) In yidghah zevir, das von Tomaschek BB. VI 200 zu av: hizvā u. s. w. gestellt wird.

Indogermanische Forschungen II 1 u. 2. 


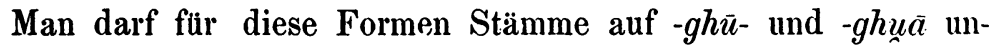
bedingt annehmen. Die germanischen Sprachen setzen *tur$g(w) \bar{o}-n$ - oder *turagū-n- (Möller a. a. O., vgl. J. Schmidt Pluralb. 74 N. 1 f., Verf. GGA. 1890, 752) voraus, eine Erweiterung der ursprünglichen Stämme auf $-g h u \bar{a}$ und $-g h \bar{u}-:$ g. tuggō, aisl. tunga, ahd. zunga, as. tunga, ags. tunge u. s. w. Das Lat. hat lingua, sei es lautgesetzlich (vgl. l. levis, lacrima, larix, olère, Brugmann Gr. I 369) oder mit volksetymologischer Anlehnung an lingo entstanden aus älterem l. dingua (bei Mar. Vict. $1,9,17 ; 26,2)$. Im Abg. języ-kz, pr. insuwis - über den Verlust des Anlautkonsonanten s. Bezzenberger BB. III $134 \mathrm{f.}^{1}$ ). Alle diese Formen weisen unzweideutig auf idg. *dnghū-, *dnghua hin, d. h. sind im letzten Teile völlig identisch mit den Formen der arischen Sprachen.

Es wird dann die Aufgabe sein, den ersten Teil der arischen und europäischen Wörter mit einander zu vereinigen. In Übereinstimmung mit Bartholomae will ich als Anlaut für die arischen Formen einen Sibilanten voraussetzen. Ein arisches *š̆ghū- oder *š̆ghvā aber kann meiner Meinung nach für ur-

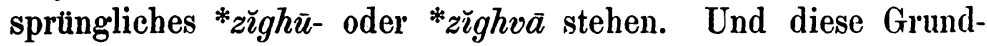
form wird sogar besser die faktischen Formen erklären als

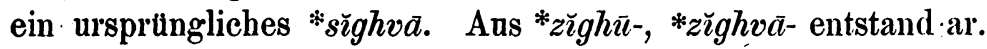
*žžhhu-, *zǔžhvã. Wie nun *svašura-, *smašru- u. s. w. zu s. švášura-, šmás̆ru- u. s. w. wurden, so wurde völlig analog

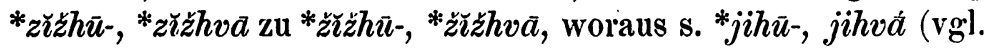
Osthoff Perf. 494 ff., 503 f., Bartholomae Ar. F. III 37 N.) Aus *jihu entstand durch assimilatorischen Einfluss des $\bar{u}$ der letzten Silbe - und vielleicht durch volksetymologische Anlehnung an die reduplizierte Wurzel $h \bar{u}$ - $h v \bar{a}$ - 'rufen' - s. juhtu-. Dass aus *zižhva u. s. w. ir. hizvā u. s. w. - vielleicht durch *sižhva- — entstand, darf nicht befremden.

Wie lässt sich nun ein (vor)arisches *zighvā aus einer Form mit n erklären - wie sie die europäischen Formen an die Hand geben - , d. h. warum entstand nicht ar. *zažhvā?

Beispiele für die Entwickelung $z n$ - zu $z \check{\imath}$ - (statt $z a$-) habe ich nicht vorzubringen. Wenn wir aber bedenken, dass $z$ oft

1) Lit. lëżùvis steht entweder ursprünglich oder volksetymologisch in nächster Beziehung' zur Wz. leigh- 'lecken', wie auch air. ligur (i. tenga Corm. 26), arm. lezu (Hübschmann Arm. St. 32). 
eine palatalisierende Einwirkung auf Vokale zu haben pflegt - so z. B. in s. $\bar{e} d h i$ von *azdhi oder ${ }^{*} z h i$ (Thurneysen KZ. XXX $351 \mathrm{ff}$.), kiyēdha aus *qiinnzdhá, mëdhá- aus *mnzdha- oder *mazdha u. s. w. (Brugmann Gr. I \& 476, 591); oder im (Lat. und) Gr., Thurneysen a. a. 0., u. s. w. - so wäre es nicht befremdlich anzunehmen, dass $z n$ - zu $z i$ - statt $z a$ - hat werden können, auch wenn man nicht andre Beispiele erwähnen kann; die Lautverbindung war eben ganz selten ${ }^{1}$ ). Es ist kaum ein gewichtiger Einwand gegen meine Annahme von $z n$ - zu $z i$-, dass die iran. Sprachen Formen wie (hazdyät, dazdi) mazdah aufweisen; denn $z n$ - hat natürlicherweise anders behandelt werden können als -ngz-, um so viel mehr, als $z$ in der Verbindung -nz- sekundär sein kann (aus urspr. $s$ ). Zudem konnte aber, wenn nicht das Angefuhrte stichhaltig sein sollte, die Entwickelung $z n-\mathrm{zu} z i-$ in einer noch ursprtinglicheren Lautverbindung begrundet sein.

Diese ursprunglichere Lautverbindung hat nach Ausweis der europäischen Sprachen ${ }^{*} z d n g h \bar{u}$-, ${ }^{*} z d n g h u a$ sein mussen. Ganz wie $d n$ - im Lit. Sl. $n$ - geworden ist (Bezzenberger a. a. O.); so ist bei diesen Formen in den arischen Sprachen $d$ verdrängt worden, eine Verdrängung, die ubrigens in der Stellung von $d$ zwischen $z$ - und $-n$ - hinreichende Begrtindung findet. Wie nun häufig in den idg. Sprachen Formen mit und ohne anlautendes $s, z$ wechseln, so hat man ganz naturlich eine idg. Parallel-

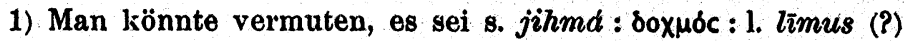
$=j i h v a ́$ : dingua : lingua. - Dass unter bestimmten Bedingungen $d i$ oder $d i(d y)$ im S. - doch wahrscheinlich nur dialektisch - durch gegenseitige Assimilation von $d$ und $i, i$ palatale Affrikata werden konnte (s. Bugge KZ. XIX 422. J. Schmidt KZ. XXV 1. 68. 149), ist wohl kaum zu leugnen, vgl. das von Bloomfield Am. Journ. of Phil. YII 482 ff. (vgl. jetzt JAOS. XIV, XLIII) herangezogene upajî́kā 'a kind of ant' (AV. II 3, 4; VII 100, 2) = upadīka M. v. F. (Cat. Br. XIV 1, 1, 8; Tāit. Ār. V 1, 4; 10,6); man könnte auch an volksetymologischen Kontaminationsbildung aus zwei Wörtern upa$d \bar{\imath} k \bar{a}$ und upajîhvike $\bar{a}$ (RV. VIII 102. $21=$ VS. XI 74. TS. IV 1, 10, 1) denken, während Bloomfield das letztere als Volksetymologie, durch upajík $\bar{a}$ veranlasst, betrachtet; upajihivike a wiederum könnte durch Volksetymologie entstanden sein statt eines *upadihvika (vgl. $d \bar{e}^{-}$ $h i k \bar{a}$ 'ein Insekt, das die Erde aufwirtt', upadèhitè 'eine Ameisenart' bei Hēmac, zu Wz. dheigh- in déhat, dìhāná-, teîxoc, g. dìgan), vgl. Grill Hundert Lieder des AthB Bediat 81 you by fINSEAD 
form *dnghua $\bar{a}^{*} d n g h \bar{u}$ - vorauszusetzen. Und dies ist die Form, die in den meisten europäischen Sprachen auftritt.

Ein direktes Zeugnis aber für die Existenz eines ursprünglichen Anlautes $z d$ - möchte ich im Altirischen finden. Wie bekannt, geht im Inlaut in den keltischen Sprachen $-z d$ - aus urk. $-z d$ - oder $-z d h$ - - durch $d d$ in $t$ über, wie z. B. aus air. net, kymr. nyth aus idg. "nizdo- (l. nīdus), air. cretim (s. çrád-dadhāmi), brot aus idg. *bhrozdho- u. s. w., s. Stokes K.-S. B. VIII 338; Thurneysen KZ. XXVIII 15̌2; Keltoromanisches 47; W. Meyer KZ. XXVIII 166 f.; Brugmann Gr. I 521; 595. Man hat keinen Grund anzunehmen, dass $z d$ - im Anlaut anders behandelt worden sei, so lange keine Beispiele eine andre Entwickelung bezeugen: d. h. idg. *zdn$g h \bar{u}-{ }^{*} z d n g h u \bar{a}$ muss eine urkeltische Form *tngu- und *tng(u)a ergeben haben. Diese selben Stämme finden sich freilich nicht; doch begegnet ein Wort, das unmöglich davon getremnt werden kann ${ }^{1}$ ), nämlich tenga, tenge $\mathrm{Wb} .23^{\mathrm{d}}$. Sanct. h. 18 (mo thenga, s. ZE. 255, Windisch Ir. Gr. § 136). Nach Stokes BB. XI 88) wäre tenge auf einen Stamm *tengaiat- zurückzuführen. Ich sehe keine andre Möglichkeit zur Erklärung des irischen Anlautes und die von mir angenommene Grundform gibt, scheint mir, über die arischen Formen die vergleichsweise beste Auskunft ${ }^{2}$ ).

Von andern Wörtern, die möglicherweise mit idg. *zdnghü* zdnghua zusammenhangen können, weiss ich keine sichern Beispiele. Doch will ich folgendes bemerkt haben. Die Wurzel ist vielleicht als zdengh- anzusetzen. Wenn wir uns nun vergegenwärtigen, dass die Zunge aus einem Grundbegriff Spitzigkeit, spitzig sein hergeleitet werden kann, wobci besonders auf gr. $r \lambda \omega \hat{c} c \alpha$ im Verhältnis zu $r \lambda \omega \hat{x \in c}$, $r \lambda \omega x i c$ 'Spitze' hinzuweisen ist, so könnte man vermuten, dass g'. stiggan, an. stinga 'stechen' u. s. w' auf die genannte Wz. $z d e n g h$ - 'spitzig sein' zurückgeführt werden müsse. Man braucht dann nicht stiggan mit der idg. Wz. steig- in críw, l. insti-

1) Das Wort für entlehnt - woher? - anzunehmen findet sich kein Anlass.

2) Über die Möglichkeit, hierher das schwed. dialektische (Dalarne) ti'eta (wo ' Nasalvokal bedeutet) 'zwitschern' u. s. w. zu stellen s. Noreen Ark. f. n. fil. IIl 19. 
gare, ahd. stehhan (vgl. Kluge $\mathrm{Wb}$. unter stechen, stange; Fick III-344) zu vereinigen, eine Etymologie, die sowohl durch das Nichtvorhandensein einer Wurzelform steik-, als aus andern Gründen unwahrscheinlich ist. Aus der parallelen Wurzelform dengh- könnte man besonders an. tange, vgl. land-zunge, herleiten, vielleicht auch ahd zanga, an. tọng, nhd. zange, u. s. w. Doch sind diese vielleicht besser zur idg. Wz. $d a_{x} n k-$ (১ó́kvw u. s. w.) zụ stellen (vgl. Schade a. a. 0.).

2. Skr. sabhä und Verwandtes.

S. sabhá bedeutet in der vedischen Zeit "Versammlung der Dorfgemeinde, das Gemeindehaus, wo diese Versammlungen stattfanden', dann 'geselliges Lokal für die Männer, Spielhaus', in späteren Schriften auch 'Gerichtshof', s. z. B. RV. VI 28,6; VIII 4, 9; AV. VII 12; sabhēya- 'im Rate brauchbar' RV. I 91, 20; 'schlagfertig. (Sänger)' RV. II 24, 13, s. Zimmer Aind. Leben $172 \mathrm{ff}$. Von diesen genannten Bedeutungen ist freilich 'Versammlung der Dorfgemeinde' die ursprünglichste. Die älteste Bedeutung aber mag 'Zusammenfassung von verwandtschaftlich Zusammengehörigen' u. dgl. als Kollektiv, oder 'Stammesverwandtschaft' als Abstraktum sein. Da nun der Stamm auf Agnation gebaut war, bedeutete sabhd 'Stammgenossenschaft' und 'Stamm', insbesondere wie er in corpore auf der Volksversammlung auftrat, d. h. 'Gemeindeversammlung'. Unter Voraussetzung dieser Bedeutungsentwickelung und der angenommenen ursprunglichen Bedeutung hat man mehrere Wörter damit zusammenzustellen. Zuerst möglicherweise av. Habaspa-, ap. aba-carish (Fick $\mathrm{I}^{4} 326$, vgl. jedoch Spiegel Ap. Keilinschr. ${ }^{2} 205$; jetzt noch anders F. Muller Wien. Zs. IV 308), A. Kuhn KZ. IV 370 f. hat mit Recht g. sibja, as sibbia, ags. sibb ${ }^{1}$ als Ableitung yon der dem s.

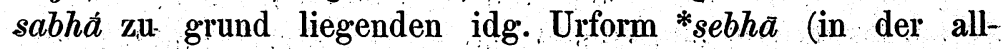
gemeinen ursprünglichen Bedeutung 'Zusammenfassung der Stammverwandten') erklärt: sibja muss also der Bildung. nạch "das zu den Stammverwandten gehörende, Stammverwandtschaft überhaupt' bedeuten (vgl. Kluge Wb. 319). Weiterhin sind dazu gestellt (so z. B. von Fick Wb. 1 795; II 677) abg. sebro 'Bauer als Teilhaber an der gemeinsamen Feldmark', lett. sebrs 'Freund, Kamerad', lit. sebras 'Teilhaber, Gefährte',

1). An. Sif, vgl. Grimm Myth. ${ }^{4}$ (I) 257 , Osthoff Quaest. myth. 19. 
lit. sébris 'Nachbar'. Alle diese Wörter weisen unzweideutiģ auf ein idg. *sěbho- zurtick, mit Ablautswechsel sēbh-: sěbh-, ein durch zahlreiche Beispiele in den indogerm. Sprachen gut verbürgter Vokalwandel. Dass abg. sebrz auf idg. *sěbhro-, lit. sébras auf idg. *sezbhro- beruhen sollen (Osthoff Perf. 491), wird wohl kaum jemand annehmen, abgesehen davon, dass das Lautgesetz, wonach dies möglich sein soll, sonst keine Stuitze hat. Dagegen scheint die Verschiedenheit zwischen lit. sebras und abg. sebrr kaum auffallender als die zwischen $\tilde{\eta} \pi \alpha \rho$ : s. yákrọt, l. jecur, ñccuv : l. sequius, g. qeens : an. kvenna, an. kváda : s. jatu-, d. kitt, ags. cwidu u. s. w. (vgl. Bugge KZ. XIX 428; Kluge Festgr. an Böhtlingk 60) u. s. w. Hierzu stellt nun auch, wie mir scheint nicht ohne Berechtigung, Baunack

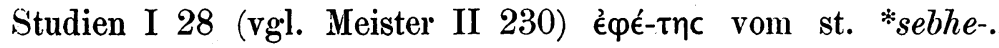

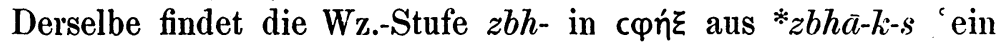
in der Sippe, im Schwarme lebendes Tier', dann 'Wespe', eine

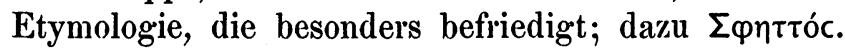

Die schwache Form findet sich auch anderswo. Bei ihrer Betrachtung werden wir auch einer noch ursprïinglichern Grundbedeutung von idg. *sebhä begegnen.

Es ist hinreichend bekannt, dass 1. suus 'eigen' im Plur. $s u i$ 'die eigenen, die seinigen oder ihrigen' bedeutet, besonders 'die einer gewissen Gemeinschaft angehörigen', und naturlicherweise am ehesten einer solchen, die durch Verwandtschaftsbande geknuipft ist: "sui sunt qui ad nos pertinent, nostri, vel cognatione et affinitate vel amicitia aut studio partium vel officio"; Cic. 14 Att. 12: Octavius, quem sui Caesarem salutabant, Philippus non u. s. w. Und sehr häufig erscheint der Reflexivstamm in den idg. Sprachen in Verbindungen und Weiterbildungen, die in nächster Beziehung zur l'amilien-, Haus-, Stamm- oder Parteigemeinschaft stehen. So ist an. Sviar, wie mir scheint richtig, von Noreen (Föreläsningar öfver urgerm. judlära 23) aus *suēe-ino- pl. *sue écioses 'die Seinigen, zum Stamm gehörigen’ erklärt worden. L. saepes (unsicher), gr. cүкóc, ahd. sweiga, ahd. suein 'Hirte, Knecht', ags. swän 'Hirte', an. sveinn 'Knecht, Diener, Knabe' enthalten auch, nach Bugge BB. XIV 66, den Reflexivstamm ${ }^{1}$ ). Ich bringe nun

1) Andre Wörter, worin der Reflexiv-Stamm auftritt mit der Bedeutung: von 'eigen, zur Familie gehörig' u. s. w., sind z. B. idg. *sue-sðr- (über *sŏr, *sĕr 'Weib' vgl. Bezzenberger Gött. Nachr. 
auch (vgl. Verf. b. Torp Beitr. z. L. v. d. geschl. Pron. 15) den oben besprochenen Stamm sěbh- $z b h$ - in unmittelbare Bezie-

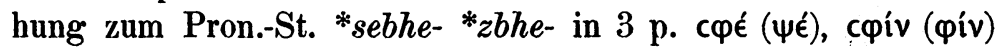

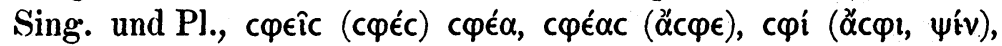

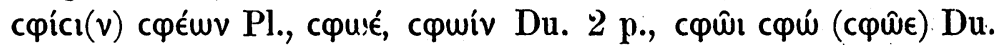
3 p., l. sebei, abg. sebě. Dieser Stamm *sebho-, s(e)bhe- ist ebenso aus den Pron.-Stämmen *se- (*so-) und *bho- (*bhe-) komponiert wie *seuo-, *s(e)ue- aus *se-(*so-) und *uo- (*ue-) und ${ }^{*} t e u o-,{ }^{*} t(e) u e-$ aus *te- (*to-) und *uo- (*ue-), vgl. l. tebei, d. b. *seuo-, *sue-, *suo- und *sebho-, -*zbhe-, *zbho- sind ganz parallele idg. Gebilde (s. Verf. BB. XIII 123 f. XIV 153 f.).

Somit sind wir auch in der Lage die beiden Erklärun-

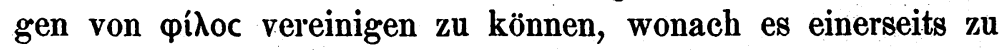
s. sabhá (Baunack a. a. O.), anderseits zum Pron.-St. сфє- (z. B. Bugge KZ. XX 41 ff.) gezogen ist (anders \%. B. Fick KZ. XVIII

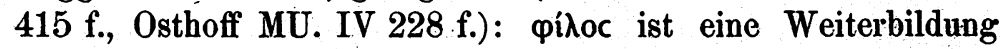
vom Pron.-St. $c \varphi$ - und die Grundbedeutung mag ' $z u$ sich, zu den Seinigen gehörig, eigen', dann 'lieb' u. s. w. gewesen sein.

Wie sabhá u. s. w. aus dem Pron.-St. so- + Suff. -bhoentstanden ist, so deute ich den (Volks)namen Schwaben, Suēbi

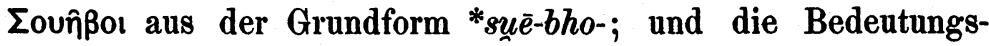
entwickelung ist dieselbe wie bei dem von Noreen gedeuteten Svīar $\left.{ }^{1}\right)$. Die Herleitung Rud. Muchs IIZ. XXXII (1888) $407 \mathrm{f}$. ist mir nicht annehmbar. - In diesem Zusammenhang lässt sich auch eine annehmbare Erklärung für d. selb, an. sjalfr, g. silba gewinnen. Einerseits haben wir abg. seli aus *se-lz-, anderseits apr. su-ba- 'selbst'. Germ. *se-l-b- nun ist nichts als der Pron.-St. se-, so- mit beiden Suff. -li- und -bho-, ganz wie $\tau \bar{\alpha} \lambda \hat{i k o c}<{ }^{*} t a-l i-q o-$ mit den beiden Suffixen gebildet

1878 271. Bugge BB. XIV 76. Brugmann C. St. IX 594. Verf. Beitr. z. gr. Sprachk. 140 f.), idg. *sue-keur- (N. *suekuèr, *suekuōr), woraus s. švišsura-, l. socer u. s. w., lit. svaine 'Schwester der Frau' = arm. keni (Hübschmann Arm. St. I 54), lit. svainius 'Bruder der Frau' (der Bildung nach mit an. sveinn zu vergleichen); weiter vgl. russ. svojákz 'Schwager, Bruder der Frau', svojaćina 'Schwester der Frau', mhd. ge-swĩe 'Schwägerin'. Aus einem vielleicht mit l. suē-tus wozu lat. svē-sco eine Neubildung ist - nahezu identischen idg.

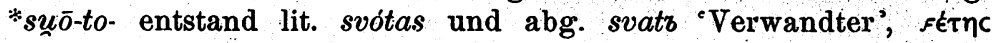

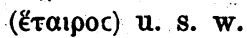

1) So jetzt mit ausführlicher Motivierung auch A. Erdmann Über die Heimat und den Namen der Angeln 96 ff. (Korrekturnote). 
ist, die einerseits in $1 . t \bar{a}-l i-s$ (abg. $t o-l i, t o-l \check{e}$ ), anderseits in abg. $t a-k z$ (aus *tā-qo-) 'solch' auftreten (vgl. auch russ. tolbko 'nur', bloss'); idg. *se-li-bho-, germ. *silba- : abg. seli: apr. suba- =

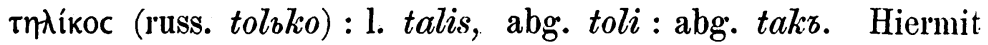
können folgende Wörter verglichen werden.

Wie der St. sue-bh- zu se-bh-, so verhält sich auch

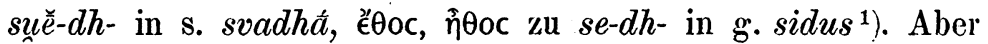
auch der mit $l$ erweiterte Stamm, der in selb erscheint, kommt in den klassischen. Sprachen vor. Ein idg. so-l- (se-l-) liegt dem s. sarvá-, ö $\lambda \circ c$, l. sollus, solus, salvus aus so-l-uo- zu grunde (s. Thurneysen KZ. XXVII 160 f.). Es bedeutete eigentlich 'für sich (abgeschlossen)', daher 'ganz'. Auch solēre gehört hierher und vergleicht sich ungezwungen mit svesco. Wie su-ba ohne $l$ zu sel-b-, so verhält sich auch das osk. sivom 'totum' (in tab. Bant. s. Zwetajeff IIID. 231, 22), d. h. sē-uom (aus sē-uo-), zu l. sollus u. s. w. Eine Grundform sē-lo-, sō-loliegt in g. sēls, l. (con-)sōl-äri, i- $\lambda \eta-\mu \mathrm{l}$ (Froehde BB. IX 119) vor; anders über sōlor sōlacium Moulton AJoPh. X 285.

\section{Skr. píbämi, l. bibo.}

Dass l. bibo direkt mit s. píbämi, air. ibim zusammenhängt, kann nicht verkannt werden; bibo ist natürlicherweise aus einem älteren *pibo durch Assimilation - im Anschluss an das gewöhnliche Reduplikationsverhältnis - entstanden. Dass diese Verba von der Wz. pö- $p^{\check{-}-}$ - schematisch als pōi (Schulze KZ. XXVII 420 f.) anzusetzen, aus $p \bar{o}-i$ - - nicht getrennt werden können, leuchtet von selbst ein. Nur ist es nicht ausgemacht, wie $b(v)$ in s. píbami (pivami) zu erklären sei.

Das idg. reduplizierte Präsens *pipōmi wurde wahlrscheinlich ursprünglich so flektiert: *pi-pómi, *pi-pósi, *pi-pó-ti; *pi-pa-ués oder *pi-p-ués, *pi-pa-thés oder *pi-p-thés, *pi-patés oder *pi-p-tés; *pi-pa-més oder *pi-p-més, *pi-pa-thé oder *pi-p-thé, *pi-pa-nti oder *pi-p-nti. Hier gibt es wenigstens zwei Formen, wo $p$ in unmittelbarer Verbindung mit tönenden Konsonanten stehen konnte, nämlich 1,3 Pl. (wie auch $1 \mathrm{Du}$.). Dies konnte im Präsenssystem ausserdem geschehen in Med. 1, 2, 3 Pl. (ev. 1 Du.) Imp. Akt. $2 \mathrm{Sg} .3 \mathrm{Pl}$. Med. $2,3 \mathrm{Pl}$;

1) Das g. swès ist entweder suce-s-o- vgl. Fick $I^{4} 578$ oder *sunedh-s-o Weiterbildung des $s$-St. in $\hat{\eta} \theta 0 c$. 
Impf. Akt. (1 du.), 1 Pl., Med. (1 du.), 1, 2, 3 Pl., im ganzen Part. Auch wenn wir die Formen nicht berücksichtigen, wo $p$ vor $m, n, n$ (u. $u$ ) zu stehn kommt, so konnten möglicherweise Präs. 2 Pl. Med. *pi-p-dhué, Imp. 2 Sg. Akt. *pi-p-dhi, 2 Pl. Med. *pi-p-dhuém, Impf. Pl. 2 Med. *(é)pi-p-dhuém, woraus ganz natürlich *pi-b-dhué, *pi-b-dhi, *pi-b-dhuém, *(é)pi$b$-dhuém - wodurch ein Wechsel zwischen $p$ und $b$ schon im idg. Paradigma entstand - im einen oder andern einzelnen Fall bewirken, dass $b$ durchgeführt wurde. Insbesondre kann die häufige Anwendung der 2 Sg. Imp. Akt. dabei wirksam gewesen sein, um ein schon idg. durchgeführtes $\mathrm{Pa}$ radigma *pibōmi u. s. w. zu schaffen.

Nun glaube ich aber, es kann als idg. Lautregel aufgestellt werden, dass die Tenues in unmittelbarer Verbindung mit Nasalen (und Liquiden?) zu Medien werden, wenigstens wenn der nächst vorhergehende Vokal unbetont war. Die Erseheinung ist mehrfach berihrt z. B. von Brugmann Gr. I $190 \mathrm{f}$; Zimmer Nom.-Suff. $a$ und $a 288$ f.; Osthoff MU. IV 328 f.; P.-BrB. VIII 268 f.; Perf. 548; Kluge P.-BrB. IX 180 f., vgl. KZ. XXVI 98 f.; Thurneysen ebd. 301 ff.; Bersu Gutt. S. 135 N. 2 u. A. Hier muss ich auf nähere Begrtindung der Regel verzichten und sie nur als Vermutung mitteilen. Wenigstens muss die Möglichkeit zugegeben werden, dass unter gewissen Bedingungen im Paradigma von *pipōmi vor $m, n$, $n$ das $p$ als $b$ hat auftreten können. Somit wären die Bedingungen fur das eventuelle Auftreten von $b$ in hohem Grade erweitert, und es scheint mir wahrscheinlich, dass das durchgefuhrte $b$ in s. $p i-b a-m i, 1$. bibo diesen Formen zuzuschreiben ist.

Trifft nun meine Erklärung von píbāmi-bibo zu, so fällt, scheint mir, Schulzes Annahme von pōi- als 'Wurzel' d. h. als die letzte einfache Einheit, zu welcher wir zurückzugehen vermögen ${ }^{1}$ ). Ich vermute von einer ursprünglichen

1) Doch kann Schulze insofern im Recht sein, dass wirklich ein $p \bar{o} i$ - als Wurzel - obwohi als eine sekundäre - gelten kann. Ich läugne durchaus nicht die Möglichkeit, dass die neuentstandene Wurzel $p \bar{o} i$ - unter gewissen, von J. Schmidt und Schulze erörterten, Bedingungen sekundär zu $p \bar{o}$ - hat werden können. Und es wäre doch wohl nicht unmöglich, auch von diesem sekundären p $\bar{o}-$ aus meiner Erklärung von $b$ in pibam $i$ - bibo zuzustimmen. 
Wurzel $p \bar{o}$ - beispielsweise zwei Konjugationsklassen *pi-pō-mi und *pōio (*pōi-mi). Aus der letzten Konjugation wäre pōi(p̌̃-) als Wurzel abstrahiert (vgl. Verf. De deriv. vb. contr. $177 \mathrm{ff} .172 \mathrm{ff}$.).

\section{Skr. lakșati 'sehen, schauen' u. s. w.}

Die Vermutung Kluges (Festgr. an Boehtlingk 60), dass idg. $l_{\bar{o}} \mathrm{im} \mathrm{S}$. bestehen bleiben soll, ist unmöglich aufrecht zu erhalten. S. lōká-1) (ulōká-) ist mit lit. laükas, l. lücus u. s. w. zusammenzustellen (Fick I 757), nicht mit l. locus, das ausserdem auf *stlo-co-zurückzuführen ist (vgl. Corssen Krit. Beitr. $461 \mathrm{f}$. Ausspr.

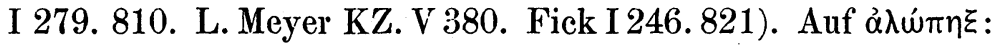
s. lōpä̌̆a- ist nicht zu bauen (vgl. Hübschmann Arm. St. I 17. Bartholomae BB. X 294). S. lōkatē, lōcatē, lōkayati, lōcayati

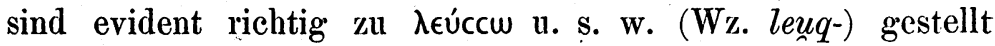
(Fick I 199. 757; übrigens vgl. v. Bradke ZDMG. XL 3う̌1). Zur Bedeutung' vgl. d. scheinen oder abg. zъréti zъrja 'sehen, blicken ', zorø 'visus' : abg. zorbnz 'glänzend', zorja 'Glanz', lit. žeriu žerèti 'glänzen' (vgl. J. Schmidt KZ. XXV 133 u. Fick II 561). D. lugen, mhd. luogen, ahd. luogèn ist mit Recht von Kluge Wb. u. lugen zu air. lagat 'Auge' gestellt ${ }^{2}$ ). Statt sie mit lokayati zusammenzustellen, muss man vielmehr diese Wörter mit skr. lakș̆ati, -e 'sehen, merken', lakṣ̌ayati 'bezeichnen' u. s. w., aus idg. lagh-s-oder logh-s-, verknipfen. $\mathrm{Ob}$ auch die von Froehde BB. III 8 hierzu gestellten griechischen Wörter ヘoziac u. s. w., damit zusammenhängen, bleibe dahingestellt.

\section{Skr. khadgá- und Verwandtes.}

Air. claideb, kymr. cleddyf, cledd ist mit Recht mit an. hjalt, ags. hilt, ahd. hëlza (Fick I 529. II 58. 324. III 72) zusammengestellt $^{3}$ ). 0 . Frankfurter hat die genannten irischen Wörter zu s. khadgá- gestellt (KZ. XXVII 222). Beide Zusammenstellungen sind richtig. Nur muss hervorgehoben werden, dass die keltischen Wörter einerseits und die arischen und germanischen anderseits auf verschiedene Wz.-Formen, (s)k(h)eld- und $(s) k(h) l e d-$, zurückgehen, wie besonders

1) Wie Kluge übrigens schon Bopp Gloss. ${ }^{3} 337$.

2) Anders, mir sehr unwahrscheinlich, über ahd. luogēn Per'sson Wurzelerw. und Wurzelvar. 218 N. 1.

3) Siegfried (Stokes) KSB. V 18 nimmt als Suffix tva an. 
air. claideb aus *k(h)ladago- : s. khadgá- aus *k(h)ald(ə)go- (vgl. Fortunatov BB. VI 216) darthun. Es ist von einer Wz.-Form $(s) k(h) a_{x} l a_{x} d-$ auszugehen, woraus je nach der Betonung entweder $(s) k(h) \breve{a}_{x} l d$-oder $(s) k(h) l \breve{a}_{x} d-$ geworden ist. Das Verhältnis wird durch folgende Beispiele beleuchtet: s. gräbha- : gárbha-, av. urvāza : s. valha-1), av. myastra : s. maid- in médin-, s. vyá$d h a^{-}$: s. vēdha-, av. vae $d h a_{-}$, vgl. Bartholomae ZDMG. XXXVIII 132, möglicherweise av. thwyā- 'Furcht' : av. dvaēthā-, $\delta F \in 1-$; s. myakș̌- (in ápamyakșá u. s. w.) : av. maēkant- (vgl. Geldner KZ. XXIV 144 f.), suméka- (Windisch Festgr. 144 f. Th. Baunack Stud.I 378), s. bhrájas : s. bhárgas, $\beta \lambda \eta \times \eta ́$, ahd. klagan : s. gárhati, s. vyáthati : av. vaêtha- (vgl. Geldner BB. XV 259); s. väghát- 'Beter, Opferer', 1. voveo : av. aojaiti

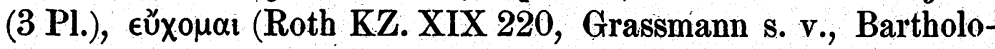
mae Ar. F. I 11 u. A.), s. vakşana-, av. vakhshat u. s. w. : aủzw 1. augeo u. s. w. Dass ich in diesen Fällen von zweisilbigen Basen, etwa *ueiedh-, *euegh-, ausgehe (dass ich sonach in ähnlichen Fällen nicht z. B. griechische Metathese, Anaptyxis oder Prothese annehme), habe ich mehrmals angedeutet (De deriv. vb. contr. 92 ff. BB. XIII $115 \mathrm{f}$. XV 308 ff. in wesentlicher Übereinstimmung mit Fick GGA. 1881, $1425 \mathrm{ff}$.). Es war dieser Gegenstand ausführlich erörtert in meinem noch nicht veröffentlichten II. Excurs zu De deriv. vb. contr. Inzwischen sind mehrere hierher gehörige Probleme behandelt worden von Kretschmer KZ. XXXI 390 ff., von Bartholomae BB. XVII $91 \mathrm{ff}$., und Persson Wurzelerw. u. Wurzelvar. $94 \mathrm{ff}$., bes. $218 \mathrm{ff}$., wo mehrere Beispiele der Art wie s. bhrajjas : bhárgas verzeichnet sind.

6. Skr. tujati, tunjati und Verwandtes.

Dies Wort, mit der Bedeutung 'schlagen, stossen, antreiben, fördern' u. s. w., hat noch keine annehmbare Anknüpfung gefunden; denn die von Fick I 823 . III 343 gemachte Zusammenstellung mit 1. tango usw. leuchtet nicht

1) Av. urvāzishta- Y. 36, 2, s. vrājá- : av. varez(-ēna Y. 36, 1), s. varj-, Th. Baunack Stud. I 354. $363 \mathrm{ff} .445$ (anders Bartholomae Hdb. 221. Geldner KZ. XXVII 586. XXVHI 259. 409), av. khrapaitĩ : s. kalpatēe (Geldner KZ. XXVII 238); av. rap- : s. arpáyāmi (vo:I Geldner Stud. z. Av. I 29. KZ. XXVII 256 f.), s. rakṣ- (rákşas u. s. w.), av. răsh- (rāshayañhē $):$ s. arš- 'beschädigen' u. s. w. 
ein (vgl. J. Schmidt Voc. I 153). Nur eine von den frïheren Zusammenstellungen lässt sich halten, nämlich die mit an. stokkv, ags. stocc, ahd. stoc u. s. w. (Kluge s. stock). Hierzu stelle ich nun auch das schwedische stuka, ursprünglich wohl 'stossen, schlagen', jetzt etwa 'überwältigen' in körperlicher und moralischer Hinsicht. Wir haben sonach eine schwache Wurzelform stug- mit 'beweglichem' $s$ - gewonnen ${ }^{1}$ ).

Ich werde jetzt zu konstatieren versuchen, dass die Normalform wahrscheinlich als stueg- anzusetzen ist. Zunächst begegnet hier die Frage, wie die idg. Verbindung stu- (oder st $\iota)$ in den Einzelsprachen erscheint. Diese Frage kann ich hier nicht mit ausführlicher Motivierung beantworten. Ich behaupte nur, dass daraus unter gewissen Bedingungen $s p$ - wird und hoffe dies bald durch Beispiele beweisen zu können. Ist meine Behauptung richtig, so kann man daran denken $\psi \epsilon ́ r \omega$ aus * spegō aus *stuēgō zu deuten. Die Bedeutungsentwickelung von 'stossen" zu 'tadeln' ist leicht verständlich. Die Bedeutung des lit. spik-ti, pri-spik-ti 'ermahnen' vergleicht sich z. B. der des schwedischen stöta $p a$ in der Bed. 'erimnere' (einen). Ich verweise auch auf 1 . vituperare, das vielleicht aus *vitituperare zu deuten ist. Das erste Element, auch in vitiligare in derselben Bedeutung (Loewe Prodr. 5), ist mit vitium (vgl. s. vyathă 'fehlgehen') identisch und tuper-ãre enthält einen $s$-St. (vgl. тútoc n.) aus der W\%. stup- in s. tópati, tumpáti (pra-

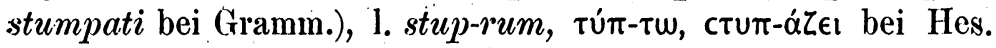
u. s. w. ${ }^{2}$ ).

1) Auch die von Bezzenberger BB. XII 241, 17 genannten Wörter sind hierher zu ziehen. - Lit. tüzgiu 'stosse' ist schon von Fröhde, BB. X 300 \%u tun̂játi gestellt; und Bezzenberger ebd. (vgl. I 169) vermutet als Grundform *tunzgiu, wonach s. tuñj- aus tuñjj- entstanden wäre. Es hängt dies mit der Frage zusammen, wie solche Fälle wie abg. brézgb (: lit. brëkszta), lit. resgiù règzti (vgl. s. rújjuFortunatov Sāmaveda-Āraṇyaka-Samhitā 121. Bezzenberger BB. I 68), blizgëti (vgl. Zimmer Nom.-Suff. $-a$ und $-\bar{a}$ 68) u. s. w. zu erklären sind (vgl. Bezzenberger ZGLS. 81 N. 5 f.). In den meisten dieser Fällen liegen gewiss Wurzeln auf $-z g$ (ev. $-s k$ ) vor. In andern dürfte eine lautliche Entwickelung wie die von Osthoff Perf. $33 \mathrm{f}$. für s. ubjáti u. s. w. angenommene wahrscheinlich sein. So könnte wohl tunzg- aus *tu-n-g-zg und dies aus tu-n-g-sq- (Inchoativbildung zu $t u-n-g-)$ entstanden sein.

2) v. Sabler KZ. XXXI 280 deutet vituperare aus dem Präfix $v i$ - und tuperare, was, wie J. Schmidt bemerkt, durch die Glosse 
Die Wz. stueg-, aber ohne $s$-, erscheint noch in mehreren bisher nicht gedeuteten griechischen Wörtern. Dass das von

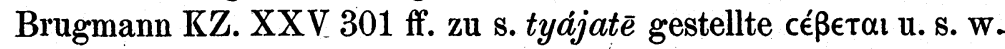
lautlich und möglicherweise auch begrifflich - céßouar etwa 'stosse von mir' - hierher gezogen werden kann, will ich nur erwähnt haben. Dagegen ist $c \in \beta-$ u. s. w. aus tuegsicherer in folgenden Fällen.

Es gab eine Ableitung auf -(u)en(o)-. Eine Fem.-Bildung würde nun urspr. so heissen: *túg(u)nă Gen. *tug(u)nás.

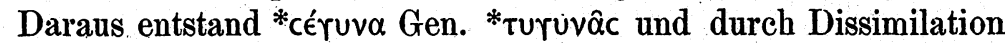
* trruvâc. Sei es lautgesetzlich, sei es durch Einfluss von *ć́ruva entstand daraus *crruvâc. Die schliessliche Form ist cırúvn - wozu círūvoc, círūvov - 'Wurfspiess', eine Bedeutung, die besonders gut zu unsrer Wurzel passt, vgl. s. túj- 'treibend, schnell', Instr. tuja 'Antrieb, Anstoss, Angriff'. Wahrscheinlich ist eine -ĭă-Bildung * $c^{\prime} \gamma^{v}-(v) v-j \breve{\alpha}$ anzunehmen. Auch Formen mit aus $g$ entstandenem $\beta$ kommen vor, nämlich cıßúvn cíßuvov. In welchem Verhältnis der Name eines skythischen Volkes

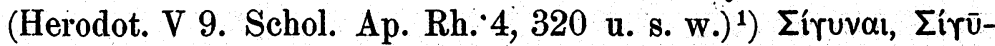
vor Eíruvvor Eírivvo hierzu steht, ist fraglich. Entweder ist er rein griechisch und von *círūva hergeleitet oder doch hierdurch volksetymologisch verändert.

Die griechische Herkunft der Wörter cırúvn cıßúvn wird sowohl durch die Form derselben als auch durch die weitere Verwandtschaft verburgt.

Es ist eine wohlbekannte Thatsache, dass $n$ - und $r$ Stämme im selben Paradigma mit einander wechseln. Eine idg. Bildung wie tueg- $(u) r(-t)$ Gen. $t u(e) g(u)-n$-és wäre demnach wohl denkbar. Der $n$-St. ist durch die Fem.-Bildungen *círūva und *cıßuvva gesichert. Zum $r$-Stamm ziehe ich folgende. Wörter. Zunächst stellt sich das Adj. coßapóc in der Bed. 'keck, beweglich, rasch, fluchtig' u. s. w. doch weit besser hierher als zu der von Brugmann behandelten Wz. tieg-. Besonders ist für diese Anknüpfung auf den von schwacher Wurzelstufe anscheinend mit derselben Ableitung gebildeten s. Namen Túg-ra-, wohl 'gewaltig, hinstürmend', hinzuweisen. Die Wz.-Forn

vitiligant unwahrșcheinlich ist. - Die Erklärung Bréals MSL. V $27 \mathrm{f}$. scheint mir zu gesucht.

1) Vgl. De Lagarde Ges. Abh Br $_{2}$ tgh ht to you by | INSEAD 


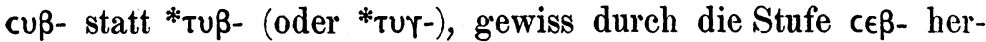
vorgerufen, erscheint in mehreren Namen, unter welchen $\Sigma$ v́ßăpıc M. Mannsname, F. Frauen-, Quellen- und Stadt-Name, Namen, denen schon von den Alten Bedeutungen wie 'rasch, keck, stürmend' beigelegt wurden (s. Suid. s. coß $\alpha \rho o ́ c, ~ \Sigma u \beta \alpha-$

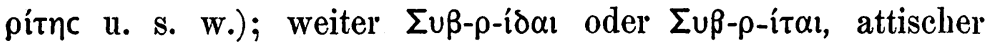
Demos. In den genannten Wörtern. ist $v$ in cuß- regelmässig, weil kein $v$ nachfolgt. Wo dies aber der Fall war, erwarten wir eine Dissimilationsform mit 1 ; so in $\Sigma_{i} \beta u ́ p-\tau-\alpha c$ (Vok. Theokr. V 5. 72. 74). Dies wird von den Schol. z. St.

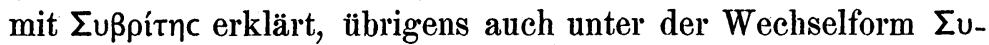

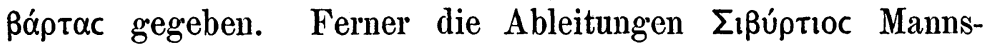

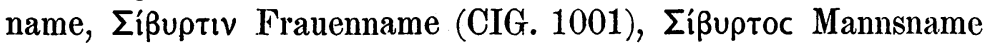
und Stadtname.

Alle diese Namensformen sind sehr wichtig, weil sie allem Anschein nach das $t$, das in Stämmen wie s. yákrnt beliebig auftritt, in den Ableitungen bewahrt haben.

Zur Klarlegung der lautlichen Verhältnisse im Anlaute dieser Wörter mag hier die Behandlung einiger andrer griechischen Wörter folgen.

Man hat längst das gr. cíkuc, cı́úa 'Gurke' mit abg. tyky f. 'cucurbita' vereinigt und zwar ganz mit Recht ${ }^{1}$ ). Von einer Wurzelform tuek- (oder tueq-) stammt cєK- in $\Sigma \epsilon-$

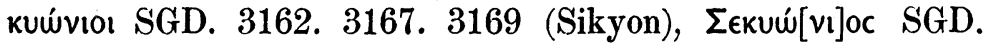

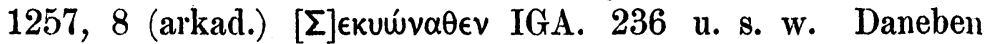

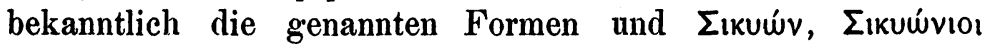
(z. B. IGA. 70 nach Fabricius Jahrb. d. arch. Inst. I 178. SGD. 3169 auf Münzlegenden). Die beiden Wurzelformen erklären sich durch Annahme eines Paradigma tuéku- : tukéu-

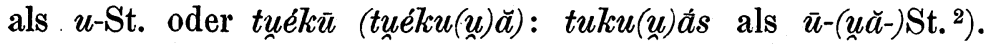

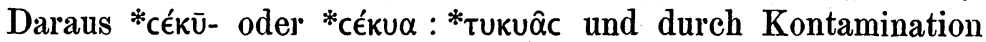
und Dissimilation cıкúa, bei Hes. cєкoúa (vgl. G. Meyer ${ }^{2} \S 88$ ). Ob cıkú $\alpha$ ein Fem. auf $-\breve{a}(-\partial)$ oder $-i \grave{a} \breve{~(-i \partial)}$ ist (vgl. Prellwitz GGA. 1886, 763 ff.), ist hier gleichgültig.

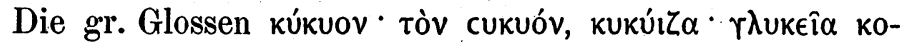
$\lambda o ́ k v v \tau \alpha$ bei Hes. dürften, mit l. cucu-mis zusammengehalten,

1) Kretschmer freilich (KZ. XXXI 335) leugnet jetzt, wie mir scheint, ohne hinreichenden Grund, diese Zusammenstellung.

2) Eine solche Stammform ist ausser durch сєкú $\alpha$ und vielleicht cíkūc auch durch abg. tyky erwiesen. 
falls sie zu cíkuc u. s. w. gehören, wohl durch Assimilation von $t \mathrm{zu} k$ nach dem zweiten $k$ ihren Anlaut erhalten haben. Diese Assimilation kann damit verglichen werden, dass von

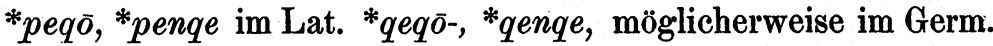
aus *qetuōor- ein *qequōr- (Kluge Konj. 102. P.-BrB. VIII 517 ff.; dagegen J. Schmidt Anz. f. d. Alt. VI 123 f., vgl. Noreen Ark. f. n. fil. VI 317 f.), entstanden ist.

\section{Skr. udára- und Verwandtes.}

S. udára-, udará- N. 'Bauch Magen', av. udara-M. id. (vgl. s. udarin̄i 'schwanger', Pott KSB. II 41): s. an-üdara'ohne Bauch' MBh. XIV 1305: lit. vedaras 'Magen' sind Ablautsformen einer Wz. uesd- (J. Schmidt Pluralb. 205).

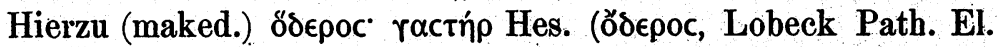
$410)^{1}$ ). Ob diese Wörter mit Fick $I^{3} 208.4128$ zur Wz. uěd- 'nass sein, quellen' gehören, ist nicht zu entscheiden, obwohl es nicht ganz unwahrscheinlich ist ${ }^{2}$ ). Ein Thema *uéd(e)r(-t)*u(e)d-nés ist auch nicht abzulehnen. Auf einen $n$-St. bin ich geneigt folg. hes. Glossen zu beziehn: údveîv

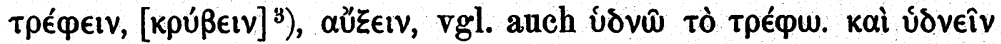

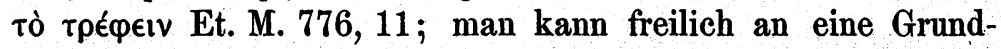
bedeutung 'wässern' denken, aber ebensowohl an die verdauende Thätigkeit des Magens. Dieser letzten Anschaung

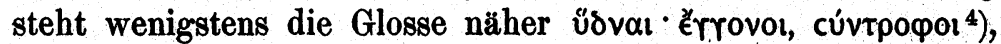
vgl. $\dot{\alpha}-\delta \in \lambda \varphi o ́ c$, g. kalbō, an. kalfr, s. gárbha- 'Brut der Vögel,

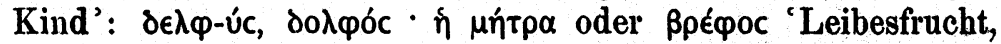
Kind': schwed. krdfva 'Kropf', oder ags. cild 'Kind': s. jarta-,

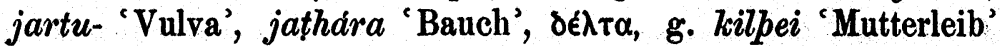
n. s. w.

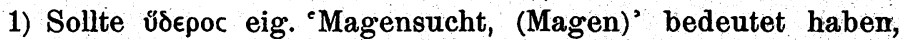
so ist wohl schon idg. aus sachlichen Gründen dies Wort mit *udōr, ưdwp assoziiert.

2) Man kann auch an Zusammenhang mit eue-, ǚe, $\check{u}-{ }^{e}$ anziehen, kleiden, hüllen' denken (vgl. òmentum 'Netzhaut, ind- $\bar{u}$ mentum 'Gewand', ind-ü-cula, ind-uere u. s. w. Fick II, 34).

3) Diese Übers. hat wohl auf ein ganz andres Wort bezug, vielleicht, wie M. Schmidt z. St. vermutet, auf ein maked. kuঠveîv. -

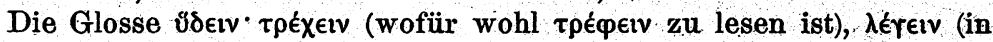

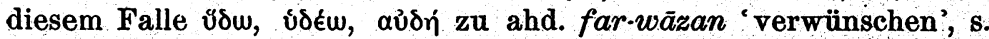
vádati u. s. w.) bei Theogn. (An. Ox.) 19, 27 ist wohl zu údveîv zu ziehen.

4) Anders, mir nicht wahrscheinlich W. Schulze quaest. hom. 55: 


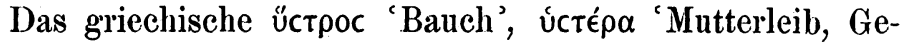

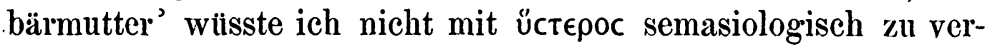
einigen (wie meist geschieht, vgl. Pott $\mathrm{I}^{2}$ 637, zulet\%t Brugmann Gr. II § 75 S. 179). Ich stelle es zu s. ŭdara- und nehme eine Bildung * ŭd-tero- an, parallel mit *ŭd-ero-. Beide fussen urspr. auf Lok.-Bildungen *üd-er und *üd-t-er (parallel mit $* \breve{u} d$-en, *ŭd-t-en). Über das eventuelle Erscheinen des $t \mathrm{~s}$. Verf.

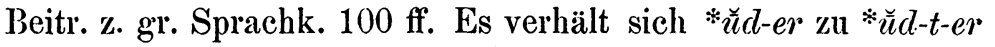

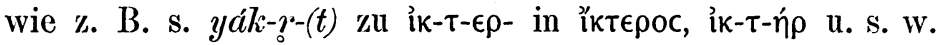

Es liegt jetzt sehr nahe hierher auch l. üterus und üter 'Schlauch' zu ziehen - denn dass diese Wörter zusammenhängen ist doch sehr wahrscheinlich, üter-: üter $=$ s. udair ${ }^{\prime}(-$ aus (an-)üdara-. L. üterus wưrde früher zu g. qipus, laus-qiprs

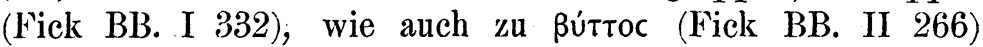
gestellt. Nach Bersu Gutt. 140 wäre diese Ansicht ungerechtfertigt. Obwohl es mir scheint, dass für diese Behauptung keine ganz entscheidenden Gründe vorliegen, so scheint mir doch die Trennung recht ratsam zu sein. Und die lat. Wörter lassen sich unschwer mit úctépa vereinigen. Ich nehme idg. Parallelbildungen *ŭd-tero- und *ŭd-tro-. Aus diesem entstand (nach de Saussure MSL. VI 246 ff. Kluge Pauls Gr. I 336. Noreen Urg. judl. 122. Verf. P.-BB. XV 229) schon idg. ŭtro-, durch dessen Einwirkung aus *üdtero eventuell ein * ütero- ${ }^{1}$ ) entstand. Dies ist im Lat. zur Herrschaft gekommen. Dazu wirkte besonders der unthematische r-St. üter ütris mit, aus *üdtēr *ütrés.

Möglich wäre nun freilich, bei ŭter- von $\breve{u}$ - als Wur\%elform und -ter-als Suff. auszugehen, in welchem Falle man die Form $u \breve{e} d-$, $\breve{u} d$ - in $u \breve{e}+$ Wurzelerweiterung $d$ aufzulösen hätte. Eine Parallele hätte man zum Beispiel in den Worten für 'Wasser, nass' $u \bar{e}-$ (in s. véa-ri u. s. w.): uete-d- in an. vaitr ags. woet u. s. w. Diese Annahme ist jedoch für uterus in anbetracht von úcté $\rho \alpha$ weniger einleuchtend.

Immerhin darf als unsichere Vermutung hervorgehoben werden, dass auch sonst die Wz.-Form (uế-:) $\bar{u}$ - vorhanden ist. Es könnte nämlich sein, dass dies $\bar{u}$ - in ved. $\dot{u}$-vadhya-n. 'Inhalt des Magens und der Gedärme' (úvadhyam udárasya RV. I

1) Vgl. übrigens die Nom.-Form uter Caecil. ap. Non. 188, 15; uterum n. Pl. Aul. 10, 7, 10 vgl. s. udara- n. 
162, 10; úbadhya- AV., üvadhyagōha- 'der Ort wo úvadhyaverborgen wird' Vāitān.) steckt. -vadhya- möglicherweise 'was auszustossen, -drücken ist', vgl. ex-scrē-menta, screa (zu Wz. sq(h)er-; vgl. ckŵp, šákrtt) u. a. m.

\section{Skr. asthat 'sogleich'.}

Das Wort kommt im RV. nur X 48, 10 vor. Es ist sehr verschieden erklärt worden. BR., Boehtlingk, Grassmann, Lanman Nouninfl. 447 erklären es als Adv. mit der Bed. 'sogleich'. Ludwig Rigv. II 604 f. V 477 f. und Bergaigne Rél. véd. II 459 sehen darin eine Form des Wortes ásthi asthnás 'Bein'. Es heisst dort gōpá némam avir asthá krnōti und - wird von Ludwig übersetzt: 'der Hirte [Indra] macht die andre Hälfte [vortra] durch den Knochen sichtbar' indem er die Situation auf I 84, 13 bezieht, wo es heisst: Indro Dadhīcó asthábhir vọtrány ápratișkutah̆ jaghāna navatîr náva "Indra tötete mit Dadhyane's Knochen [nach Bergaigne "le soma manifesté, sorti du monde invisible pour coopérer a la victoire d'Indra, peut-être sous la forme de l'éclair, de la foudre, qui est en effet souvent identifiée au soma", vgl. Rél. Ved. II 99. $458 \mathrm{f}$. III 49] unaufhaltsam die neunundneunzig Vitra (desselben)'. Er fasst demnach asthd́ als Instr. Man hätte dann auch für das Ski. eine Form des einsilbigen Stammes *osth-1), wie in av. as-ca Nom.-Akk., astas-ca Gen. Sg., astãm Gen. Pl., azdébīsh Instr. Pl. (s. de Saussure Mém. 226. Mahlow L. V. 80. Bartholomae Ar. F. II 122. BB. XV 38. J. Schmidt Pluralb. 109. 266. Verf. BB. XVIII 25 f. u. s. w.). Bergaigne a. a. 0. (vgl. jetzt auch Hillebrandt Ved. Mythol. I 337 f.) fasst asth $\dot{c}$ als Akk. Plur. und ubersetzt demnach 'le gardien manifeste l'autre sous forme $d^{\prime} o s^{\prime}{ }^{2}$ ), in welchem Falle 'le gardien' freilich Indra ist, 'l'autre' den soma (Dadhyanc) bezeichnet, wogegen Ludwig darunter die vrtráni erblickt. Wer oder was mit némasmin ('chez l'un' als Gegensatz zu 'l'autre') im vorhergehenden Pada gemeint sei, ist demnach schwer zu erraten, wohl eben der Dadhyán̂c (Ludwig sieht darin den Mond).

1) Natürlich nicht aus *asthiā wie Ludwig Rigv. $\mathbf{V} 477$ vorschlägt.

2) D. h. astháa wäre N. Pl. N. gleich náăà u. s. w. (s. indessen J. Schmidt Pluralb, $82 \mathrm{ff}$, der fréilich eine solche Form nicht kennt, von seinem Standpunkt aus sie auch nicht anerkennen würde, es sei denn als N. Pl. eines $r$-Stammes).

Indogermanische Forschungen II 1 u. 2. 
Dann aber ist von den vortráni nicht die Rede und man gewinnt an RV. I 84, 13 für die Deutung asthá als 'mit Bein' oder 'sous forme d'os' keine Stütze. - Noch anders Pischel

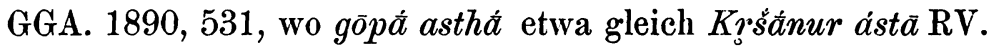
IV 27, 3. IX 77, 2 vermutet wird. Er bezieht die Stelle nicht auf die Legende von Dadhyan̂c wie Ludwig und Bergaigne, sondern auf die Legende vom Raube des Sōma (s. Pischel Ved. Stud. I $206 \mathrm{ff}$.), und sieht in astha die von ihm aufgestellte Wz. asth- 'werfen'. Dies aber gibt Pischel selbst als unsichere Vermutung aus. Ich glaube auch nicht, dass es eine von $a s$ - 'werfen', ursprünglich verschiedene Wz. asth- gegeben hat." Die Gründe werde ich anderswo zu geben suchen.

Sollte hier wirklich keine Form von idg. osth- 'Bein' vorliegen, so scheint mir die von BR. statuierte Bedeutung ansprechend. Die Bildung ist leicht verständlich. Es ist die instrumental-lokale Form eines Wurzelnomens sthā. Es ist um so unbedenklicher ein solches anzunehmen, als es sonst in einer andern Zusammensetzung und in der selhen Form vorkommt, nämlich prati-ștthá RV. X 73, 6. a- möchte ich für ein präpositionelles Element erklären, nämlich n, die schwache Form zu *en 'in'. Analoge Bedeutungsentwickelungen lägen dann vor in l. illico aus *in slocö, fr. sur-le-champ, d. auf der Stelle, norw. u. schwed. (dial.) ista(d) 'jüngst,' kürzlich' u. a. m.

\section{Skr. kukṣsi- 'Bauch'.}

Die etymologische Erklärung dieses Wortes hängt davon ab, wie man die Entstehung des $k s ̣$ im Skr. begrenzt. Bekanntlich nahm man allgemein an, dass s. šşs d. h. ar. $\check{s} \grave{s}$

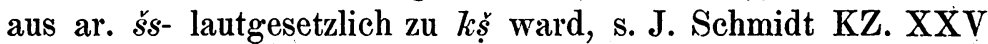
118 f. Pluralb. 222 n. 1 f. 417 . Brugmann Grundr. I § 401 \$. 299. $\S 556$ S. $411 \mathrm{f}$. Diese Annahme geht Hand in Hand mit jener, dass $\hat{k} s$ durch urar. $s ̌ s ̌$ zu $k s \grave{s}$ ward. Diese letztere ist bestritten von Ljungstedt Anm. till det starka präterit. i germ. språk 134 und Bartholomae KZ. XXIX 573 ff. Sind die Einwände dieser Forscher berechtigt - und mir scheinen sie es zu sein - dann ist das Hauptargument für die Ansicht, dass $\left(i, u, r, r^{\circ}\right)+s+s$ zu ar. šs lautgesetzlich d. h. ar. oder indisch zu $k s ̧ s$ werde, weggeräumt. Zuerst hat Ljungstedt a. a. 0. 139 die Möglichkeit - ohne sie selbst freilich unbedingt an- 
zunehmen - hervorgehoben, dass die Entwickelung von şs zu $k s s^{2}$ z. B. in $d v \bar{e} k s \underline{s} i$ auf Analogie beruhen könne: vaik̂- vesștum,

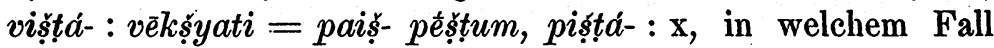
$\mathrm{x}=$ pékşsyati. Bestimmt hat Bartholomae a. a. 0. Stud. z. idg. Sprachgesch. I 52 ff. diese Analogieerklärung behauptet und ausfuhrlich motiviert. Mir scheinen seine Gruinde stichbaltig. Es ist demnach meines Erachtens nicht ratsam s. koş aus šss zu erklären, wenn nicht daneben analogiewirkende Formen im selben Formbildungssystem vorkommen. Es ist mithin nicht wahrscheinlich, dass $k u k s s ̧ i$ - aus ${ }^{*} k u s ̧$ ssi- zu erklären sei.

Wir können demnach die Wz. nicht als qus- ansetzen ${ }^{1}$ ). Aber man kann auch nicht von einer Wz.-Form qeuq- ausgehen, wie Fick $I^{3} 303 .{ }^{4} 190$ es thut. Freilich wäre es möglich, wenn man nur das Skr. berulcksichtigt. Aber vergleicht man, wie es Fick richtig thut, av. a-fratat-kushìs F. Pl. (d.h. kushi-); so wird es unmöglich. Dies kann - ausser aus *kus-s-i-, was durch die oben gegebene Entwickelung ausgeschlossen ist - nur aus $\hat{k} s$ entstanden sein (Hübschmann KZ. XXIII 398 ff. ZDMG. XXXVIII 428 ff. J. Schmidt KZ. XXV 119 ff. Bartholomae Gãthās 87. Hdb. § 147. Ar. F. I 22\%). II 57. 177. Collitz AJoPh. VIII 214 ff. Brugmann Grundr. I \& 401). Aus $q(h)$ ist $s h$ nur in der Verbindung $q i$, ar. $c y$ geworden (Hubschmann KZ. XXIV 353. XXVII 106. ZDMG. XXXVIII 430. Geldner KZ. XXVII 228 Amn. 257. Dr. Yasht 24. J. Schmidt KZ.

1) Wir trennen billigerweise folgende Wörter ab: kucbc, lat. cunnus, falls aus *leus-no- (Aufrecht KZ. IX 282. J. Schmidt ebd. XXV 126). kucóc wăre demnach aus *kuc-co-, aus einem 8 -St., hervorgegangen. Es ist aber nicht völlig ausgemacht, dass die genannten Wörter aus kus- zu erklären sind. So nimmt Froehde BB. XIV 100. XVI 196 die Wz. kut- an in l. cut-ur-nium "vas quo in sacrificiis vinum fun-

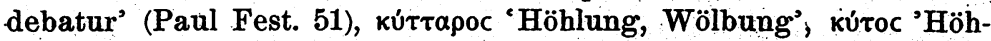
lung, Gefäss, Urne', lit. kutỹs 'Beutel'; l. cunnus sei aus *kut-no-,

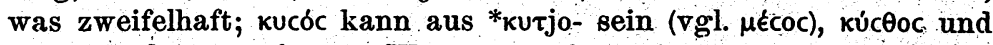
кúctic sind aus mehreren Wurzeln erklärt: 1. von qeut- oder keut(Froehde a. a. 0.), weniger wahrscheinlich; 2. qeudh- (Brugmann Gr. I \& 469 S. $340 . \& 507$ S. 373 . § 536. 538 S. 396. \& 552 S. 406 . Bartholomae Ar. F. I. 176. Kluge P.-BrB. IX 153. W. Meyer KZ. XXVIII 166) oder sqheudh-; 3. qeus- (Grimm Myth. IIB 922. J. Schmidt KZ. XXV 166. XXVIII 180 N. 1. Fick I 51. 303. 537 u. s. w.).

2) Das dort gegebene Beispiel av. väsha- 'wagen' wird freilich Ar. F. II 35. 40. 48 anders erklärt. 
XXV 68. Pluralb. 418. Brugmann Gr. I $\S 448 . \S 476$ S. 551. Bartholomae Hdb. § 102. BB. VII 190. Ar. F. II 52. 129. 134. III 20, vgl. Hdb. § 117. Ar. F. II 13 f. 133 . Brugmann Gr. I $\S 475)$. Das av. kushi- wäre dann aus einer Fem.-Form */cuci gen. *kushyās zu erklären. Für eine solche Form aber gibt es keinen Anhalt, am wenigsten wenn wir, wie wohl richtig, s. kuksssi- vergleichen. Wir bleiben demnach für beide Sprachen

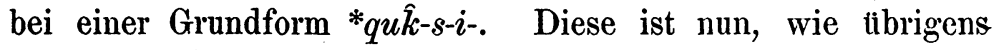
schon Froehde BB. XVI 196 gesehen hat, mit lit. kuszỹs 'Vulva', das von s. susși- 'Spalt, Loch' zu trennen ist, zu verbinden. Wurzel ist demnach qeûk-, die ich in s. kós̆a- 'Behälter, Fass, Kufe, Kasten', lit. káuszas 'Gefäss' wiederfinde. Obwohl dies schon Grausmann Wb. s. (kuśs) ausgesprochen hat, scheint es nicht unangemessen, nochmals ausdrücklich hierauf hinzuweisen, wenn Fick noch $\mathrm{I}^{4}$ 27. 181. u. kousos zwei Wurzeln vermischt. Ebenso sicher wie eine Wz. qeu-s-in l. cūria, ahd., as., ags. $h \bar{u} s$, g. (gud-)hūs (vgl. Grimm Myth. I ${ }^{3}$ 922, Corssen I, 353 f. u. a.), vielleicht auch in s. $k \overline{o s s} a-$ mit denselben

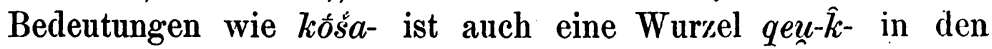
oben behandelten Wörtern. S. kóša- und kōsóa- können gar wohl zwei ursprünglich verschiedene Wörter mit ähnlichen Bedeutungen sein. Dadurch und durch Lautähnlichkeit kann leicht Vermischung eingetreten und die speziellen Bedeutungen der einzelnen Wörter ausgeglichen worden sein.

\section{Skr. ká- 'Wasser'.}

Dass das Subst. ká- M. Göttername (Prajapati-, Brahman-, Vişnu- u. s. w.) mit dem Pron. ká- zusammenhängt, ist mehrfach angenommen. So habe man aus dem kásmäi dévàya des Refrains RV. X 82, $1-9$ einen Gott $K a$ - schon im RV. misverständlich herausphilosophiert. Dies mag richtig sein. Als Neutrum aber scheint das Wort $k a \dot{a}-m$ doch andern Ursprungs zu sein. Es bedeutet 1. 'Freude, Glückseligkeit', 2. 'Wasser', 3. 'Kopf'. In der Bed. 'Wasser' lässt sich das Wort etymologisch begreifen. Es kommt Māitr. S. I 10, 10 vor. Das Wort dürfte die schwächste Stufe des lat. aqua sein; idg. é $q$-:(ə)qé-. Grassmann vermutet, dass in kè-nipá- RV. X 44, 4 als erstes Element $k a^{-}$' Wasser' enthalten ist, und übersetzt es 'in Wasser gebietend'. Ludwig Rigv. II 248. V 201 fasst es als 'Steuerruder' und denkt an Entlehnung aus dem semit. kanaf 'Flügel', 
was doch wohl allzu gewagt ist. Vergleicht man akē-nipá‘in der Nähe Schutz gewährend' oder dgl., so sieht kēnipáwenigstens indisch aus, obwohl die eigentliche Bedeutung unklar bleibt. Dass in der späteren Sprache kēnipata- 'Steuerruder' bedeutet, ist bekannt. - Soviel, wenigstens scheint nicht unannehmbar, dass s. $k a^{\prime}-$ 'Wasser' etymologisch in Beziehung zu l. aqua u. s. w. steht.

\section{Skr. tadit u. s. w.}

S. tadít als Gegensatz zu dürè sán RV. I 94, 7 ubersetzen die meisten mit 'in unmittelbarer Nähe seiend, dicht daran', Ludwig I 301 dagegen mit der in der späteren Sprache gewöhnlichen Bedeutung 'Blitz'. So auch II 342 taditas RV. II 23, 9 als 'Blitze'. Sei dem wie ihm wolle: ist für RV. eine andre Bedeutung zu statuieren als flir das klassische Skr., was mir nicht nötig scheint, so ist doch das ved. tadit (talít): mit klass. tadit etymologisch identisch. Und es ist wohl nicht zu bezweifeln, dass die hauptsächlich im Epos und im klassischen Skr. erscheinenden Wörter von Wz. täd-, nämlich tdada-AV. +, tatăda C., tạdayati E., tādaka-, tădya-, tădana- E. +, -tad̆in-, tädañ̄ya-, tädayitar- C. damit wurzelverwandt sind (s. Whitney Wurzeln 60). Ich suche in diesen Wörtern die abstrakte Grundbedeutung 'stossen'. Auch wird die Imp.-Form vitậhi (šátrün) RV. X 180, 2 hierhergezogen mit der Bed. 'zerstosse, zersshmettere'. Ludwig V 283 zieht sie zu taják, tajat 'stracks, soeben, mit einem Schlag, gleich' mit dem Z Zusatze 'Verlust der Palatalität'. Eher könnte man an Wz. tarh- in trộédhi 'er zerschmettert' (vgl. J. Schmidt KZ. XXIV 319. XXV 62. Bartholomae KZ. XXVII 351 N. 2 f. 360.364. Ar. F.I 14. Brugmann Grundr. I \& 404. 478), trinhát, tatarha trạha- denken und annehmen, dass ar. *taržhdhi zu *tarżdi zu tädhi geworden sei. Am wahrscheinlichsten aber gehört diese Form zur hier behandelten Sippe und ist aus *taddh (nicht aus *taddhi mit Benfey GN. 1874,367 = Kl. Schr. II 174 und Grassmann W.b. u. tad-) zu deuten.

Die Wz. $t a d-$ nun erkläre ich aus ar, tald-, entstanden durch Reduktion einer zweisilbigen Base *telad, die je nach dem Grade der Reduktion ar. tald- zu *taald zu *tald zu tad- oder *tald zu tad- ergab. Diese Wz. tel(a)d-, mit fakultativ erseheinendem Anlauts-s stel( $(\partial) d$-, finde ich im Germanisohen wieder: 
Und zwar scheint mir die Bed. 'stossend' die Grundbedeutung. des ahd. stolz 'töricht, übermütig' zu sein. Entlehnung aus dem Lat. lehnt Kluge Wb. u. stolz. mit Recht ab. Grundform *stldo-. Aber auch andre Ablautsformen kommen vor, nämlich in ahd. stęlza 'Holzbein zum gehen', schwed. stylta id., die aus germ. Grundformen *staltjōn- resp. *stultjōn- (möglicherweise *steltwjōn-) ausgehen. Die ursprüngliche Bedeutung. 'stossen' tritt in skandinavischen Wörtern hervor, nämlich in schwed. stulta 'stossend (halb stolpernd) gehen'; die Stelze ist das Holzbein, womit man auf den Boden stossend geht.

Wenn ich hier ein indisches $\ddot{\alpha} \pi$. $\lambda \epsilon \gamma$. erwähne, nämlich talīdyà- AV:VII 76,3, so geschieht es nicht, weil ich eine evidente Etymologie darlegen zu können glaube. Der erste, der dies Wort sachlich und etymologisch zu deuten versucht hat, ist Bloomfield, der es AJoPh. XI (1890), 328 ff. sorgfältig erörtert hat. Bloomfield kommt zu dem zweifelsohne richtigen Resultat, dass $t a-$ lĩ $d y \dot{a}^{-}$' the bottom of the body' im Gegensatz zum Scheitel bezeichnen muss. Näher bestimmt er talizdyà- als 'the sole of the foot' und identifiziert es mit dem klassischen talahrdaya- 'die Mitte der Fusssoble', das er nach den medizinischen šăstra noch mehr spezialisiert 'the sole under and behind the fourth and fifth toe', und das er als eine volksetymologische Umdeutung des talîdyd anzusehen geneigt ist. Alles dies ist sehr wahrscheinlich: Nur kann man darüber zweifelhaft sein, ob talìdyà- urspringlich 'die Sohle' bedeutet hat. Obwohl sogar eine evidente Etymologie die bestimmte Bedeutung eines Wortes in einer bestimmten Zeit ohne andre Kriterien nicht angeben kann, so kann sie doch die Richtung, in welcher die Bedeutungsentwickelung vor sich gegangen ist, andeutell. Es kann gar wohl sein, dass talädyà- in vedischer Zeit die 'Fusssohle' bedeutet hat, ohne dass die Bed. 'Sohle' zu Grunde zu, legen ist. Wollte man talĩdyà- mit ahd. Stelze gleichsetzen, so hätte man anzunehmen, dass das beiden Wörtern zu grunde liegende idg. Wort auf den Teil des Beines im allgemeinen, womit man geht, Bezug gehabt hat. Die Spezialisierungen wären dann im Ind. auf die 'Fusssohle', im Germ. auf ein 'Holzbein' erfolgt. Man kann formell talidyà- und Stelze vereinigen, wenn man *talad-io- resp. *taldio- als Grundformen ansieht. Die erste Form konnte s. *taľ̆dyà- ergeben die letzte germ. ${ }^{*} s$-taltja-. Das $\underset{d}{d}$ in der überlieferten indi- 
schen Form wäre dann sekundär. Man könnte aber auch für das Skr. die zweite Form annehmen: daraus *tădyà-. Dies kann einmal neben *talždyà- bestanden und in diesem die Lingualisierung des $d$ zu $d$ analogice hervorgerufen haben.

Wollte man dagegen dem von Bloomfield a. a. 0. gegebenen Fingerzeig folgen und in tal- s. tala- 'Fläche' (padatala- 'Fusssohle', vgl. ' $\eta \lambda i^{\alpha} \alpha$, lit. tile 'Diele im Kahn', abg. tolo 'Boden', ahd. dili, dilla, an. pilja von Stämmen *beloz, *biliz u. *biljōn-) ${ }^{1}$ ) sehen, so möchte ich für talīdyd̀- entweder Zusammensetzung oder Ableitung von einem zusammengesetzten Wort annehmen. Ich möchte dann -idya- aus *il-dicodeuten und $i l$ - mit der Wurzelsilbe $\check{r}-l$. in an. $i l$ (Gen. iljar) 'Fusssohle' verbinden, worüber Kluge Wb. u. eilen u. unten S. 57.

\section{Skr. bhurij- and Verwandtes.}

Die Annahme Ficks I ${ }^{3} 383 .{ }^{4} 266$ und Andrer ${ }^{2}$ ), dass dies Wort, das man mit BR. s. v. und Grassmann s. v .als 'Schere's') deutete, zur Wz. bher- 'schneiden, bohren' (in av. pairi-barerenti 'bohren', papów, l. ferio, lit. baviu, abg. borja u. s. w.) gehöre, muss nach den Untersuchungen Pischels Ved. St. I $239 \mathrm{ff}$. als verfehlt aufgegeben werden. Pischel hat unwiderleglich nachgewiesen, dass die einheimische, offenbar traditionelle Übersetzung 'Arm' (bahhu- Nãigh. 2, 4), der auch Ludwig gefolgt ist, die einzig richtige ist. Auf Grund dieser Bedeutung versuche ieh hier eine Etymologie.

Ich gehe von der Voraussetzung aus, dass die Bedeutungen 'Balken' und 'Arm' wechseln können, dass die eine in die andre leicht ubergehe. Dies scheint mir a priori wahrscheinlich, wenn man von der Bed. 'länglich runder Stamm' im allgemeinen ausgeht. Thatsächlich kommt auch

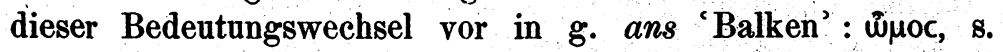

1) L. tälus dagegen ist unverwandt und aus *takslo- :taxillus entstanden (Corssen I 642, Goetze C. St. I 2, 170, vgl. Fick I ${ }^{3} 601$. Spracheinh. 238. Froehde BB. XVI 209).

2) So jetzt z. B. Persson, der Wurzelerw, und Wurzelvariation S. 18 Fick folgt und S. 283 (Nachtrag zu S. 18), ohne seinen etymologischen Standpunkt zu ändern, Pischels Auseinandersetzung zitiert. bank'.

3) So Roth RV. VIII 4, 16. AV. XX 127, 4; sonst 'Schnitz- 
ása-, 1. umerus, arm. us u. s. w., vgl. bāhú-, ahd. buog in den bekannten Bedeutungen: ags. bōj, bōh, das ausser 'Arm' auch 'Ast' bedeutet, wie auch engl. bough. Ich halte es demnach für berechtigt s. bhurïj- einerseits mit ahd. balko balcho, mlıd. balke, nhd.balken, ags. balca, an. balkr 'Gehege, Scheidelinie' mit Ablaut *bhol-g-, an. bjälke 'Balken' mit Abl. *bhel-g-, ags. bolca 'Schiffsgang' mit Abl. *bhl-g-, anderseits mit pá $\lambda \alpha r^{\prime}$ 'länglich rundes Stück Holz, Baumstamm u. s. w.' zusammenzustellen.

In formaler Hinsicht gibt es für bhurij- mehrere Erklärungsmöglichkeiten. Man kann es aus idg. bhala-g- gr. * $\varphi \alpha \lambda \alpha-\gamma-$, mit schwacher Stufe bhal-a- der zweisilbigen Wz. $b h a_{x} l a_{x^{-}}$(etwa bholo-), herleiten. Dann wäre bhurij- in der Hauptsache mit der Wurzelsilbe in ags. bolc-a identisch. Im Griechischen wäre auch ein $n$-St. im Spiel gewesen. Es ist am wahrscheinlichsten, dass sich das einst vorhandene * (aus *bhalz-g-) mit einem * ${ }^{*} \alpha \lambda \alpha-\nu-\gamma-$ (oder vielleicht ursprünglicher * $\left.{ }^{*} \alpha \lambda-n-\gamma \mathrm{zu} * \varphi \alpha \lambda \alpha \gamma-\right)$ verschmolzen hat. Das gr. * $\varphi \alpha-$ $\lambda \alpha-v-\gamma$ - oder * $\varphi \alpha \lambda-n-\gamma$ - ist derselben Art wie 1. $s$-an-gv-is gr.

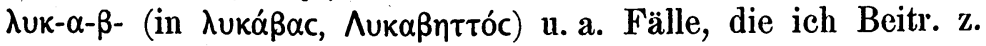
gr. Sprachk. $1 \mathrm{ff}$. behandelt habe, d. h. das $g$ ist mit dem $n$-St. statt, wie gewöhnlich, mit dem $r$-St. in einem heteroklitischen Paradigma wie s. ásrk Gen. asnás vereinigt worden. Weitere Verwandte s. Bezzenberger BB. I 256, vgl. Fick ebd.61.

Der $n$-St. ist nun auch thatsächlich vorhanden in mehreren Wörtern derselben Sippe wie $\varphi \alpha \lambda \lambda o ́ c$ aus *bh(u)al-n-ó-: Ich beziehe nämlich die hier behandelten Wörter $๘ \mathrm{u} \mathrm{W} \mathrm{W}$. bhel- oder vielleicht lieber bhuel- 'schwellen', (Verf. P.-BrB.XV 225 ff.). Die Bedeutungsentwickelung kann uber' 'das gewachsene, Gewächs, Anwuchs' zur Bed. 'Baumstamm' und 'Ast', 'Arm', oder uber 'das geschwollene, abgerundete' sowohl in drei Dimensionen (vgl. ahd. ballo, an. bọllr u. s. w.), als in zwei, (vgl. an. bolv 'Baumstamm', d. bohle, mhd. bole) geschehen sein.

Man kann sonach s. bhurij- und $\varphi \alpha \lambda \alpha-v-\gamma-$ nur damn gleichstellen, wenn man den $n$-Stamm im letzteren Worte als durch Kombination in griechischer Zeit entstanden erklärt. Ver-

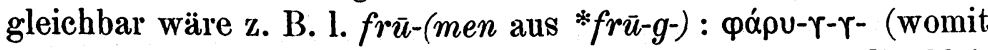
Fick BB. I 63 an. bar-k-i zusammenstellt; nur hat man die Gleichung bar-k-i : $\varphi \alpha \alpha \rho \alpha-\gamma-\gamma-$ aufzustellen) $\left.{ }^{1}\right)$ u. a. m.

1) Mit an. barki : $\varphi \alpha ́ \rho u \gamma \xi$ ist zu vergleichen mhd. slurc 'Schlund', an. slark 'Schwelgerei' : $\lambda \alpha \rho u \gamma \xi$ (vgl. Fick ${ }^{4}$ 577). 
Sonst wäre auch noch eine Möglichkeit vorhanden, bhurijaus *bhur-r-j- (idg. *bhal-r-g) durch Dissimilation des $r$-Elementes in $r$ zu erklären und darin die $r$-Form des $r-n$-Themas zu sehen; in dem Falle bhurij-: $p \alpha \lambda \alpha \gamma-\gamma-\stackrel{\text { I }}{=}$ s. ásrk : 1 . sangu-. So lange ich aber keine analogen Fälle fur diese an und für sich einleuchtende Dissimilationserscheinung vorzubringen weiss, muss diese Möglichkeit hinter der ersten Erklärung zurïckstehn.

\section{Skr. vayä 'Zweig'.}

Bekanntlich zieht man dies Wort meist zur Wz. uèei(: ueie- : ui $\mathbf{u}_{\bar{e}}$-) 'binden, drehen' ${ }^{1}$ ). Zugebend, dass diese Kombination möglich ist, glaube ich doch, dass eine andre den Vorzug haben durfte.

Ich möchte nämlich die Etymologie des Wortes Znoeig: für die des vayá normierend ansehen, wie auch schon Andre (s. Grassmann Wb. s. v.), die die Form aus *dvayd herleiten, wollen, an dieselbe Bedeutungsentwickelung gedacht haben. Ganz wie Zweig, ahd. $z w \bar{\varepsilon}(g)$ u. s. w. zu einer Ableitung. *duť-qo- (vgl.' s. $d v i-k a-$ ) von dui- 'zwei' gehört, ebenso ist vayá auf *uei-o- zu beziehen, eine Ableitung des Zahlwortes ui- (s. z. B. Fick $I^{3} 218.428$. u. s. w. ${ }^{4} 124.319 .544$. Persson b. Verf. BB. XIV 171 N. 2. Brugmann MU. V 23 f. Grund. IL § 177 s. 493. \& 293 S. 646 f. Verf. Beitr. z. gr. Sprachk. 96) ${ }^{2}$ ). Die Ableitung *uei-o- ist einem *duei-o- in s. dvayd- u. s. w. völlig analog.

Vayd kommt in allen den spezifischen Bedeutungen vor wie Znoeig. Ich erwähne besonders die Beziehung auf Verwandschaftsverhältnisse. So z. B. vayáyäs anyásyäs átithim RV. X 124, 3, wo es 'Zweig des Geschlechts, Sippe' bedeutet: Die Bedeutung 'Sprössling, Ableger' ist nach Sayyana (s. Geld-? ner Ved. St. I 277) auch für RV. I 165, 15 und für vayádvant: VI 2, 5, wo Roth vayd in der Bed. váyas nimmt, anzusetzen.

1) Zuletzt z. B. Fick ${ }^{4}$ 306. Persson Warzelerw. u. Wurzeth var. 113.

2) Übrigens gehört die Base ěu- in (e)u(e)-i 'zwei' wohl zux

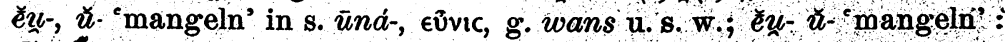

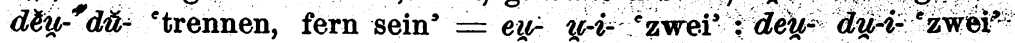
(vgl. Verf. Beitr. z. gr. Spr.-K. 148 f.). 


\section{Skr. ürú- 'Schenkel'.}

Das Gewöhnliche ist Anknüpfung an urú- 'breit'. Dies ist in begrifflicher wie besonders in formeller Hinsicht bedenklich. Ich möchte es zu l. urvum 'Krümmung des Pfluges', osk. uruvo F. TB. 'krumm' (anders Froehde KZ. XXII 255. BB. XIV 105 : zu s. vojjiná-) stellen und vergleiche den Bedeutungswechsel

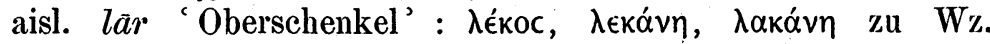
$l \bar{e}-q^{-}$'liegen' (vgl. Verf. Beitr. z. gr. Sprachk. 144) oder cké-

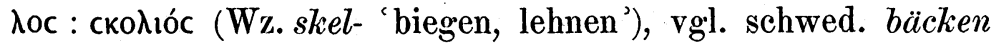
(d. becken) sowohl in der Bedeutung von 'Hüftgegend' als von 'Behälter, Reservoir'. Dieser Vergleich macht auch die Heranziehung von urvoá- 'Becken, Behälter' annehmbar. Auch der Wechsel von $u$ - und $u o$-Stamm stützt diese Annahme. Die Wz. dürfte als uĕer- anzusetzen und vielleicht mit uerin lit. veriù vérti 'öffnen und schliessen’, l. ap-erio, u. s. w. (Brugmann IF. I 174) mit der abstrakten Grundbedeutung 'biegen, drehen, winden' identisch sein. Wohin úr-dara- M. (RV. II 14, 11) gehört, das von BR. und Grassmann mit 'ein Gefäss zum Messen des Getreides, ein Scheffel' iubersetzt wird (Ludwig: 'Speicher', Sāyaṇa: kusūlam), bleibt unsicher. Man könnte vielleicht eine idg. Flexion uêr-d (wie das idg. $s a \bar{l}-d$ ' 'Salz') annehmen, wechselnd mit $\bar{u} r-n$ - in lat. $u r-n-a$, falls dies nicht für *urc-na steht. In úrdara- wäre dann die $d$ Form der Weiterbildung zu Grunde gelegt.

\section{Skr. cisyati 'werfen'.}

S. $a s$ - 'werfen' wird seit langem mit asi- 'Schwert', dies aber mit 1. ensis (wie auch boo) zusammengestellt. Ist diese Zusammenstellung richtig, so hat man ohne weiteres eine Wurzel mit Nasal anzunehmen: ásyati, asi- aus *nsiéti, *nséiIn beiden Kategorien ist schwache Wurzelform möglich oder wahrscheinlich: in der sog. IV. Klasse ist ja meist schwache Wurzelform vorhanden (obwohl ursprünglich dort wie in den Klassen I u. VI ein Akzentwechsel stattfand); und dass bei den $i$-Stämmen (wie übrigens bei andern Stämmen mit Abstufung) der Akzent zwischen Wurzel- und Suffixsilbe gewechselt hat, ist allgemein bekannt und anerkannt. Zahlreiche Spuren des ursprünglichen Zustandes sind vorhanden. Ich verweise beispielshalber : auf die den $u$-St. geltenden Erörterungen von 
Jackson BB. XVII $146 \mathrm{ff}$. In asi- ist das ursprüngliche Verhältnis zwischen Ablaut der Wurzelsilbe und Akzentlage bewahrt. Die Akzentlage erforderte als Ablautstufe des Suffixes $e ́ i$ : ursprünglich *éns- $i$ - : ${ }_{n}^{*} s-e ́ i$-.

Als Wr. stelle ich ar. ans- (idg. vielleicht ens.) auf, und identifiziere diese- Wz. mit der von Geldner Drei Yasht 135. BB. XIV 27 angenommenen Wz. ans-. Die hierher gehörigen av. Wörter sind ãsta- M. 'Feindschaft, Verfolgung, Hass', ãstãscā Y. 44, 14. ãstā 34, 8; ãstēñg Y. 46, 18, ãstāi ebd. Hierzu zieht nun Jackson Proc. May 1889 JAOS. XIV, CXXVI vazyãstra. Yt. 10, 20, und, wie auch schon vorher Bartholomae BB. VIII 220, añra (mainyu), gāth. añgra 'arg, böse', aus *ans-ra- oder *ns-ra- zu denten. Von der dieser Wz. eigenen Bedeutung 'schleudern, werfen, schiessen auf' lassen sich die Bedeutungen, die Opposition und Feindschaft ausdrticken, leicht ableiten.

\section{Skr. $r d d u$ - 'Feuchtigkeit'.}

Das Wort kommt im Sanskr. nur in den Zusammen-. setzungen rodu-dára-RV. II 33, 5. III 54, 10. VIII 48. 10, rodupé und rdū-vŕdha VIII 77 (66), 11 vor. Diese Wörter sind ausfübrlich behandelt von Benfey GN. 1875, $189 \mathrm{ff}$. = Kl. Schr. I. $316 \mathrm{ff}$. Er ist zu dem evident richtigen Resultat gelangt, dass $r d t$ - ein Subst. Fem. ist mit der Bed. 'Feuchtigkeit'. Yăska Nir. VI 4 (zu RV. VIII 48, 10, Năigh. IV 3) erklärt es

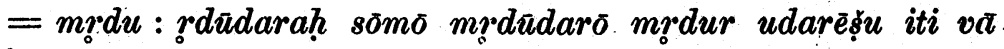
'rdädarah̆ (nämlich) Soma, der wei ch bäu chige (zartleibige), oder welcher in den Bauchen weich (angenehm) ist ${ }^{2}$ (Benfey a. a. 0. 317). So auch Sāyana zu RV. III 54, 10: rdu

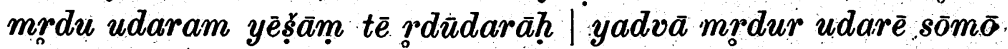

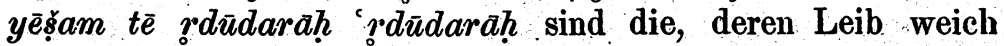
(zart) ist, oder die, in deren Leib der Somatrank weich ist's Dass diese Etymologie unmöglich ist, liegt auf der Hand (vgl. Roth Erl. z. Nir. 73). Die Erklärung Yāskảs von radūpé und räürơdhä Nir. VI 33 ist. verschieden von der des rdüdára-, aber noch unmöglicher. PW. und Boehtlingk stimmen der Erklärung Yāskas von roūudára- bei. Grassmann hat, wie Fick $I^{3}$ 24, eine andere Etymologie; er vergleicht ardra-, ắobw. Dass diese Herleitung richtig ist, hat Benfey a. a. 0. 332 ff. nachgewiesen und $r d \dot{u}-\mathrm{zu}$ av. aredvi urspr. '(at- 
mosphärische) Feuchtigkeit' gestellt (vgl. noch Bartholomae Gāth. 93. 169. Geldner KZ. XXVIII 201 mit N.2). Er hat auch richtig die Verschiedenheit der Bildung angegeben, a. a. 0. 338. Ich habe hicr an das Wort ?’dt́t erinnert, weil es derselben Bildung ist wie vadhí-, $\pi \rho \in ́ c \beta \alpha$ und sich als ein weiteres Beispiel zu den von J. Schmidt Pluralb. 54 ff. (vgl. Bezzenberger BB. VII 73) und mir KZ. XXX 402 ff. GGA. 1890, 750 ff. gesammelten reiht. Ich trage jetzt auch kein Bedenken, trotz Bury

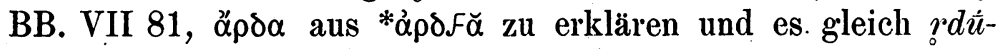
zu setzen. Zur doppelten Fem-Bildung der $u$-St. auf $-\breve{a}(-\partial)$ und -iă (-iə) s. Verf. KZ. XXX $404 \mathrm{ff}$.

\section{Skr. kévala- und Verwandtes.}

Das Wort kommt schon im RV. vor mit der Bed. 'jemandem ausschliesslich eigen (vgl. AV. X 7, 36); allein, einzig, alles andre ausschliessend, merus, pur, lauter; ganz, vollständig, gesamt, jeder, alle insgesamt'. Soviel ich weiss, sind hierfür zwei Etymologien vorgeschlagen, die eine von Fick $\mathrm{I}^{4} 18$, vgl. 375, wonach es 'zu abg. č̀-glz, cè-gzchr 'solus', l. cae-lebs aus *caevi-lebs gehören soll; die andre von O. Hoffmann GGA. 1889880 , wonach es mit notéw verwandt ist. Die letzte

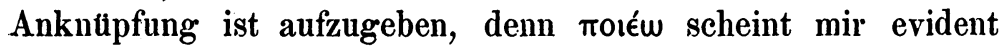
richtig von Brugmann Ber. d. s. Ges. d. Wiss. 188936 ff. Gr. Gr. ${ }^{2}$ $\S 35$ S.54 zu s. cinómi gestellt zu sein. Die erste Anknüpfung mag richtig sein. Dass die einfache Wurzel qai- lautet, beweisen die verschiedenen Ableitungen im Skr. und Abg. Es gab sicher ein *qai-uo-, die Form két-va-la- macht auch eine daneben liegende Form *qai-lo- wahrscheinlich. Diese finde ich in an. heil, heill 'gutes Vorzeichen', ahd. heil 'Gesundheit', ags. höl- id., air. cêl, kymr. coil 'augurium' von einem $s$-St. *qai-los-, der auch in ahd. heilison, ags. höelsian 'augurari' auftritt; ahd. heil, as. hèl, ags. häl, an. heill, g. hails, abg. cèlz 'vollständig, ganz'. Wurzelverwandt ist wohl auch apr. kailüstikun Akk. 'Gesundheit' (so schon Nesselmann Spr. d. a. Pr. 105, wo übrigens Verwandtschaft mit apr. kaima-, lit. këmas, g. haims angenommen wird). Hier haben wir dieselben Beziehungen zwischen 'ganz' und 'gesund', die z. B. in lat. sollus, solus : salvus (s. oben) vorkommen. Ursprüngliche Bedeutung ist wohl 'für sich abgeschlossen', dann 'ganz, intakt', und so 'integer, gesund, gut, glücklich'. 
18. Skr. addhá und Verwandtes.

S. addhá wird von BR., Grassmann, Withney $§ 1104$ als ein Adv. 'fürwahr, sicher, offenbar' gefasst und aus at + Adverbial-Suffix $-d h \bar{a}$ erklärt. Die statuierte Bedeutung ist gewiss richtig, nicht aber die Ansicht, dass es ein Adv. auf $d h \bar{a}$ sein soll. An sich könnte ja freilich ein ursprüngliches Adverb Substantiv werden (vgl. an. ef, efan 'Zweifel': g. jabai, $i b a i$, iba u. s. w. Verf. BB. XIII 124 N. 1), aber die folgenden Erwägungen machen diese Annahme in Bezug auf addh $d$ unwahrscheinlich. Schon Kern (ZDMG. XXIII 220) hat ausgesprochen, dass addhá dem. ap. azdā Bh. I 32; NRa. 43. 45 entspricht. Diese Ansicht vertritt auch Spiegel KSB. VII 94 f. Ap. Keilinschr. ${ }^{2} 86$ und behauptet entschieden, dass es ein Substantiv sein müsse mit der Bedeutung 'Kenntnis, Kunde'. Denselben Standpunkt nimmt nun auch Bartholomae $\mathbf{K Z}$. XXVIII 15 f. Ar. F. III 56 (vgl. Ar. F. I 14. Bezzenberger KSB. VIII 364. Hübschmann KZ. XXIV 17) ${ }^{1}$ ) ein und stellt zu den genannten Wörtern auch av. (gd.) azda (y. 50, 1). Er statuiert als Bedeutung 'Gewissheit, Kunde' und hat für RV. diese Bedeutung als die passendste angenommen an Stellen wie $k \delta$ addhà vēda ká ihá prá vōcad dèván aicha pathyà ká sám èti RV. III 54, 5, púnar yatt nákir addha nú vēda RV. X 129, 6, wo addha in Verbindung mit vëda als Instrumental in der Bed. 'mit Gewissheit' angesetzt werden darf ${ }^{2}$ ); vgl. hierzu auch arm. azd 'Nachricht, Kunde', das wohl mit Recht von Hubschmann Arm. St. 16 als Lehnwort aus dem Pers. gefasst wird (vgl. De Lagarde Beitr. \%. altb. Lexikogr. 16).

Es darf somit als ausgemacht gelten, dass addha, ap. av. $a z d \bar{a}$ ein Substantiv ist mit der Bed. 'Kenntnis, Kunde, Gewissheit'. Spiegel vergleicht es der Bildung nach mit s. šraddhă. Diese Auffassung sehe ich als die richtige an:

1) Die etymologische Anknüpfung Bartholomaes BB. XV 187, vgl. KZ. XXIX 326, mag an sich richtig sein. $\mathrm{Ob}$ aber adh-van-auch dazu gehört (Bártholomae BB. XVI 266 N. 1), bleibt sehr zweifelhaft. - Ưber av. azdā übrigens ganz anders Haug Gāthās II 84, wogegen mit Recht Bezzenberger KSB. VHI $363 \mathrm{f}$.

2) Dass addhá im S. als ein Adv. aufgefasst warde, ergibt sie z. B. aus dem Adj. Sup. addhätama- 'ganz deutlich, offenbar' (Āit. Ār. 47, 16), addhātamām Adv. 'ganz sicher'. 
śraddhá ist eine Zusammensetzung von šrad- 'Herz' und dem sog. Wz.-Nomen $d h \bar{a}$, das ein Nomen actionis war; und ganz so ist addhá von $d h \bar{a}$ und einem ersten Element, das noch nicht etymologisch klar ist.

S. $m \bar{e} d h \ddot{a}$ F. 'Weisheit, Verstand, Einsicht, Gedanke', mēdhäs 'sinnig, weise', av.mazda 'Gedächtnis, Erinnerung' (Bartholomae Ar. F. III 40; 55. Roth Über yaçna 31 S. 22; ZDMG. XXXVIII 437 f.; Geldner KZ. XXVII 240), ap. mazda M. (in Auramazdā (Spiegel Ap. Keilinschr. 201 f.), av. mazdāh- M. (Bartholomae Hdb. § 182). Hier kommt es für mich nicht darauf an, auf die Stammbildungsverschiedenheiten und die Flexion dieser Wörter einzugehen - es scheint am natürlichsten zwei ursprüngliche Stämme auf $-d h \breve{a}-$ und $-d h \breve{a}-s-$ anzusetzen; s. bes. Lanman On nouninfl. 549; J. Schmidt KZ. XXVI 401 f. Danielsson Gr. Anm. I51 ff. - ich will nur kurz die bisherigen Etymologieen erwähnen. Der älteste erwähnungswerte Erklärungsversuch ist von Benfey Altpersich Mazdâh $29 \mathrm{f.}^{1}$ ), wonach ein *man(a)s-dhā zu Grund zu legen sei (vgl. Geldner Stud. z. Av. I 58). Diese Etymologie galt fitther auch für Bartholomae, vgl. Ar. F. III 56, als gesichert. Jetzt dürfte sie wohl aufgegeben sein. Man deutet jetzt gewöhnlich das erste Element aus der Wz. $m e(n) d h$ - (Brugmann M. U. III $144 \mathrm{f}$. Grundr. I 476; Hübschmann KZ. XXVI 606; Bartholomae Ar. F. I 13 f.; BB. VIII 230; KZ. XXVII 353; 361. Ar. F. III 40; 55 f.; vgl. auch Danielsson Gr. Anm. I 52 N. 3). Die Wr. mendhhat die allgemeine Bedeutung von psychischer oder geistiger Wirksamkeit uberhaupt: 'kennen, erkennen, denken; geistig erregt sein; lernen'; auch die Bedeutung 'heilen' durfte nicht fern abliegen, weshalb man auch die Wörter, die diese Bedeutung haben, zur selben Wz. ziehen kann (vgl. Fick I 167; 390; 714; Curtius Et. ${ }^{5} 311$ f.; de Saussure Mém. 152; 156 u. A). S. $m \bar{e} d h \tilde{a}$ ist aus *mñdh-dha erklärt; man könnte dann auch an *madh-dha aus idg. *medh-dha denken. Jedenfalls ist idg. mendh- nur eine nasalierte Parallelform zu medh- (vgl. Hübschmann KZ. XXIII 393. Bartholomae BB. III 230). Die schwache Form dazu wäre madh-. Daraus entstand ar. $a d h-;$ nun

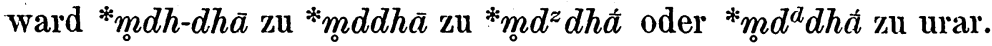
*azdhá. Dies gab zd. ap. azdā. Diesem sollte nun ein s.

1) Abh. d. Götting. Ges. d. Wiss. B. XXIII (1878). 
*ēedhá entsprechen. Wenn nun aber addhá auftritt, das nach dem vorigen von $a z d \bar{a}$ nicht zu trennen ist, so muss dies eine Neubildung sein, d. h. addhá verhält sich $\mathrm{zu}$ dem vorauszusetzenden ${ }^{*} \bar{e} d h \hat{a}$, wie s. daddhi zu $d \bar{e} h \hat{\imath}$ (von $d h \dot{a}-, d \bar{a}$-), addhi : *êedhi, runáddhi : *runeèdhi oder wie av. masti- zu *mazdi- (Brugmann MU. III 144 ff.; Grundr. I 478 Anm.; Bartholomae KZ. XXVII 351 ff.). Der Grund einer solchen Neubildung mögen entweder andere geläufige Formen mit regelrechtem $a d h$-, die vielleicht in der vọ Bartholomae $\mathrm{KZ}$. XXIX 320. 326. BB. XV 187, vgl. BB. XVI 261 N. 1. Geldner $\mathrm{KZ}$. XXX 323 N. 3, angenommenen Wz. adh-. (ah-) stecken (aus $m d h$ - verallgemeinert), oder eine stets wirkende psychologische Tendenz, Gleichheit mit anderen Wörtern (vgl. e edh- 'gedeihen': $\bar{e} d h$ - 'brennen') zu vermeiden, gewesen sein. Gegen die Annahme von anlautender Nas. son. ist vom theoretischen Standpunkt nichts einzuwenden und mehrere Etymologien sind darauf gebaut (abgesehn von dem Negativpräfix, vgl. Fick BB. I 167. V 168, VII95; de Saussure Mém. 25; Bezzenberger BB. V 168; Bury BB. VII 80. 338; Froehde ebd. 87; J. Schmidt KZ. XXVII 307; Solmsen KZ. XXIX 97; Schulze KZ. XXIX 263 f.; Bechtel Ion. Inschr. 66. Verf. KZ. XXX 414 N. 1. GGA. 1890 751 u. A.).

Die jetzt versuchte Etymologie passt besonders gut zur thatsächlichen Bedeutung von addha, azda. Die diesen Wörtern eigenen Bedeutungen 'Kunde, Kenntnis, Gewissheit' stehen den von mëdhd, mazda 'Weisheit, Gedüchtnis, Erinnerung' sehr nahe, etwa wie mentio zu mens, oder, um einer anderen etymologischen Spielerei Raum zu geben: addha, azda verhalten sich zu mēdhă, mazdā wie (Ge)wissheit zu Weisheit.

19. Skr. édhatè 'gedeiben' und Verwandtes.

In dem anregenden Aufsatz über vokalisehes $z$ im Indogermanischen hat Thurneysen KZ. XXX 352 s. édhate (ēdhati) 'gedeihen' zu s. sádhati, sädhatè gestellt (anders v. Bradke ZDMG. XL 682. 685, der die alte, von den einheimischen Grammatikein und Lexikographen stammende, Zusammenstellung mit s. rdhnotti, rdhyate zu retten sucht). So möglich mir diese Zusammenstellung von lautlichem Standpunkt scheint, so unwahrscheinlich ist sie in Bezug auf die Bedeutungen. Überschaut man die für sádhati, -tē [sädhyati, sädhnōti] statuierten 
Bedeutungen, so treten folgende als die hauptsächlichsten hervor : 'gerade aus zum Ziele kommen, seinen Zweck erreichen; zu Standekommen; - gerade lenken, schlichten, in Ordnung bringen; zum Ziele führen, zu Stande bringen; - sich fügen, gehorchen'; verwandte Bedeutungen hat das Kaus. sādháyati, tēe (s. BR. und Boethlingk Skr.-Wb.). Dagegen édhatē wird übersetzt: ' gedeihen, Wohlergehen finden, glïcklich sein (insbes. mit sukham); gross werden, um sich greifen (von Feuer und Leidenschaften ${ }^{1}$ ), anschwellen (von Wasser)'. Part. ëdhita- 'erstarkt, verstärkt, gross geworden, aufgewachsen, angeschwollen'; Kaus. èdhayati 'gedeiluen machen, verstärken kräftigen'. Mir scheint aus den Belegstellen hervorzugehen, dass die Grundbedeutungen dieser Verba ganz wesentlich von einander verschieden sind. Mir scheint die abstrahierte Grundbedeutung von sädh- 'in gerader Linie zu etwas gelangen, etwas bringen' zu sein (vgl. besonders die Bedeutungen des hierher mit Roth KZ. XIX 216 f. und J. Schmidt Vok. I 35; 181 auch von Thurneysen gezogenen $\theta u ́ c)$, jedoch die von èdh- etwa: 'schwellen, strotzen, lebenskräftig sein, wachsen'; und diese Bedeutungen lassen sich ungezwungen auf noch konkretere Anschallungen zurückführen, wie 'wohlgenährt, fett, feist sein' und dgl. Ich meine, es ist ein erheblicher Unterschied zwischen diesen beiden Bedeutungssphären. Nur in den abstraktesten Schattierungen beruhren sie sich: 'am Ziele glücklich sein, etwas glücklich vollenden' einerseits und 'lebenskräftig, glicklich sein, gedeihen' anderseits.

Deshalb halte ich es für wahrscheinlich, dass sädhati von édhate ganz zu trennen ist.

S. édhatē kann für urar. *azdhatai stehen; dies aber fï̈r ursprüngliches ${ }^{*}$ mzdhatai, idg. *mzdhétai ${ }^{2}$ ). Ich fasse die hier auftretende Wz.-Form mzdh- als schwache Form zu einem idg. $m a_{x} z d h$. . Dies $m a_{x} z d h$ erkenne ich in s. médha- M. 'Fleischsaft, Fettbrühe, kräftiger Saft oder Brühe, überh. 'kräftiger 'Trank;

1) In diesem Falle wohl mit etymologischem Anklang an $\bar{e} d h$ - 'brennen' (in, é édha-, ēdhatu'- 'Feuer' u. s. w.).

2) Andre Beispiele von s. $\bar{e} d(h)$ aus ar. $a+z+d$ (h) s. J. Schmidt KZ. XXV 60 ff. Bartholomae KZ. XXVII 351 ff. 360 ff. ZDMG. XXXVI 585. v. Bradke KZ. XXVIII $300 \mathrm{f}$. - Das von Bartholomae auf "sazdi-: zurückg'eführte sēdi- 'Erschlaffung' gehört wohl zu sásti, sasásti 'schlafen'. 
- Saft und Kraft bes. des Opfertieres, das was in ihm wesentlich und wertvoll ist; - Opfertier; - Tieropfer'; mēdhaya'aus dem Opfer hervorgegangen (Viṣnu), médhapati- und médhápati- M. 'Herr des Tieropfers', médhas N. 'Opfer' 1).

1) Von diesem médha- u. s. w. sind wohl folgende Wörter zu trennen. Es kommt nämlich ein mēdha- 'Lohn, Preisgewinn' in der Zusammensetzung mēdhá-sāti- 'das Gewinnen oder Verdienen eines Lohnes, - Preises' vor (wo jedoch die Komm. médha- 'Opfer’ sehen und das 'Empfangen oder Geben dés Opfers' übersetzen), möglicherweise mëdhayú, falls es 'nach Lohn, Preis begierig' zu übersetzen ist (Pischel Ved. St. I 103 übersetzt es 'streitlustig' in Übereinstimmung mit Sāya as Übersetzung ságrāmēcchu-; eine andre ist yajnakramanēcchu-, vgl. Bloomfield AJoPh. XII 35. Bartholomae BB. XV 7). Jedenfalls ist das av. maedha- Opfer', wie es übersetzt wird, auf eine Wz. meidh- zu beziehen und vielleicht hierher zu stellen. Ich bin um so geneigter ein zweites mêdha- in der angegebenen Bedeutung anzunehmen, als man dadurch eine Etymologie für das ahd. mèta, miata, as. mèda, ags. mèd gewinnt, das kaum mit g. mizdo, ags. meord, s. mìdhá- u. s. w., wie allgemein geschieht (vgl. z. B. Brugmann Grundr. I \& 538. 596. 621), zusammenzustellen ist. Über die vokalischen Verhältnisse, auf die ich hier nicht eingehe, vgl. Jellinek P.-BrB. XV 297. Wir gewinnen somit eine Wz. mejdh- etwa 'durch Kampf gewinnen' (vgl. Pischel a. a. O.). Diese Wurzel möchte ich auch in s. mìdhá-, 'Kampfpreis, Kampf', av. mĩzhda 'Lohn' $\mu \mathrm{ic} \theta \delta c$, abg. mzzda 'Lohn' sehen. Über dies Wort s. z. B. Benfey GN. $1874366 \mathrm{ff}=\mathrm{Kl}$. Schr. II $172 \mathrm{ff}$. Hübschmann KZ. XXIV 407. Bartholomae AF. I 16 f. u. A. Ich möchte nämlich Grundformen wie *mldh-dho- oder *midh-to- voraussetzen, woraus schon idg. *mlzdho-, ar. *mtzdha- hervorging. Ich glaube namlich, es lassst sich nachwoisen, dass das aus dentaler Explosiva entstandene $z(s)$ in derselben Weise behandelt wurde wio ursprung-

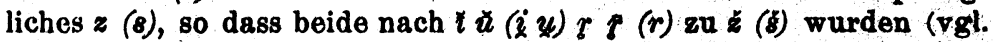
Brugmann Grundr. I \& 556 und 591). Falle wie av. verezda-, gerezdã können nicht mit Geldner KZ. XXVIII 28 n. 1, vgl. Bartholomae AF. I 12. 15. BB. VIII 224 dafür geltend gemacht werden, dass nur ursprünglich $z d$ nach $r$ zu $z d$ ward. Denn verezda-, gerezdã können ganz auf derselben Linie stehen wie s. vrddhá- (st. *vrdha-), grddhá-, vgl. die übrigen bei Bartholomae AF. I 12 ff. verzeichneten Fälle wie av. niuruzdōtemaèshva- = s. muddhá- (nicht ${ }^{*}$ rū $\left.h h a-\right)$ av. frāuruzdapayañho, uruzdipakem = s. *muddha, av. bäuzdri, dadhuwibuzda = s. Formen mit -ddh, z, hväyaozdäm s. yōddhar-. Sie alle beweisen nichts gegen die wegen frühzeitiger Lostrennung von der Verbalwurzel regelrechte Vertretung in av. mīzhda-: Als eine lautgesetzliche Form betrachto $1 \mathrm{ch}$. B. eine Bildung wie adhirüdhākárna- 'hypertrophische Ohren habend" MS. II 6, 13. III 15, 5 (vgl. L. v. Schröder Mon.-Ber. Berl. Ak 1879

Indogermanische Forschungen II 1 u. 2. 
Sehen wir uns nun nach weiteren Verwandten um. S $m \bar{e} d h$ - und $\bar{e} d h$ - stehen für idg. * $m a_{x} z d h$ - und *mzdh-. Betrach-

698), vgl. Brugmann Grundr. I § 476 Anm. 482 Anm.1. 591 Anın. 1. Bartholomae KZ. XXVII $351 \mathrm{ff}$. $360 \mathrm{ff}$. Die Zusammenstellung' von

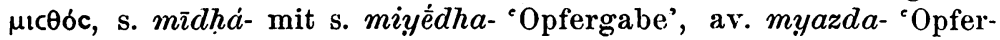
fleisch', s. Delbrück Z. f. d. Ph. I 10. Klug'e P.-BrB. VI 380 N., vgl. J. Schmidt KZ. XXV 62, Bartholomae AF. I 16 f. KZ. XXVII 353. 360. ZDMG. XXVIII 132. AF. II 130. u. A.) kann richtig sein, unter Annahme von Wechselformen meidh-: miedh-der Wurzel. Übrigens kann man s. miyé dha-, av. myazda- davon trennen und anderswohin ziehen (etwa zu s. máyas: miiaz-dh-und miaz-dh-, Fick I ${ }^{4}$ 284, oder sie wie Benfey GN. $1874366 \mathrm{ff}$. $=\mathrm{Kl}$. Schr. II $172 \mathrm{ff}$. von $m \tilde{t}-s$-(dh-) herleiten und $\mathrm{zu}$ mayatē 'tauschen' stellen). Die beliebte

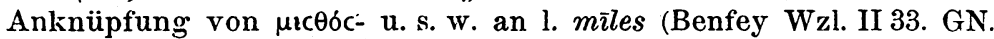
$1874371=$ Kl. Schr. II 176. Walter KZ. X 201. Kluge ebd. XXV $313 \mathrm{f}$. Bartholomae KZ. XXVII 354 u. A.; dagegen mit Recht Corssen Krit. Nachtr. 259. Froehde BB. XIV 112. XVI 208) ist gänzlich aufzugeben. Es genügt dagegen geltend zu machen, dass idg. $z d h$ (oder $d \approx d h)$ im Lat. zu st, gr. $c \theta$ wird. L. mīles gehört zu l. mill.e, $\delta-\mu i \lambda$-i $\alpha$, s. milati 'sich vereinigen mit, sich einstellen, zusammenkommen' (vgl. Fick I ${ }^{8}$ 177. 723. III 235. Stokes BB. XI 293, anders über milia Thurneysen KZ. XXX 35̄3).

Dass auch die Wz. meidh-, etwa 'aufrichten' oder viclleicht 'spitzig sein', mit to- oder dho- Suff. erweitert worden ist, beweist av. mizhda 'Nagel' (in baêvare-mizhda- 'mit zehn tausend Nägeln') wie auch s. $m e \bar{e} h h i-\mathrm{M}$., $m \bar{e} d h \bar{\imath} \mathrm{F}$. (ein weiteres Beispiel für die Fem.Bildung eines $i$-Stammes; die Gramm. fassen $m \bar{e} d h \bar{\imath}$ als Fem. zu mèdha- Pāṇ. IV 1, 41 G.; andre Beispiele s. Liebich Pāṇini 132 ff.), wohl nicht durch sekundäre Lingualisation, zu erklären, sondern aus ${ }^{*} m e i d h$-ti- neben *meidh-i- (wie z. B. vrrt-ti- neben vrt-i-). Die Bildung *meidhi- liegt vor in s. mēdhi M., -i F. gleichbedeutend mit mèthí, mēthī 'Pfeiler, Pfosten, insbes. ein Pfosten in der Mitte der Tenne, an welchen die Ochsen gebunden werden, ein Pfosten zum Anbinden des Viehes überhaupt'. Hiermit wurzelidentisch ist wohl mēdhá RV. I 88, 3, das Sāyaña durch yajnān, BR., Grassmann und Ludwig' (Rigv. II 291. V 235) durch 'Lanzen' oder 'Lanzenschäfte' geben (dagegen wohl nicht médhās RV. III 58, 2 als 'Opfersäulen' mit Grassmann, sondern mit Sāyaṇa als yajnāh oder mit Ludwig Rigr. IV 50 als 'heilige Weisheit und ihre Produkte' zu übersetzen). Mit diesen Wörtern wurzelverwandt ist wohl an. meidr 'Stange, Schlittenkufe'. Ob nun diese Wurzel meidh- (oder meith-) mit der in s. $m \bar{\imath} \not h a^{\prime}-$ 'Kampt' u.s. w. identisch ist, lässt sich nicht entscheiden. Möglich wäre dies durch Annahme einer Bedeutungsentwicklung 'Stangen, stechen, streiten, kämpfen - wetteifern um den Lohn, Preisgewinn' u. s. w. Zur selben Sippe dann auch av. maédha-. - Andre Bedeutungsentwicklung (resp. Etymologie) bei Benfey a. a. 0 . 
ten wir nun $d h$ als sog. Wz.-Determinativ und substituieren statt seiner ein paralleles Determinativ $d$, so entsteht idg. $m a_{x} z d-$. Diese Wz.-Form hat auch v. Bradke KZ. XXVIII $300 \mathrm{f}$. erkannt in s. médyati, médate (nur mèdátām) 'fett werden', mèdayati ' fett machen', mēda- M. Fett, médana- N. 'Mastung', médas N. 'Fett; Fettleibigkeit', médas-vant- 'fett' (TBr. 2, 4, 4, 9), mēdurá- 'fett (Cat. Br. 5, 4, 3, 19); — dick, dicht, voll', mëdya- 'fett; - dick, konsistent' u. A., weiterhin ahd. mast, ags. moest 'Mästung', ahd. mast, ags. gemoest 'fett, gegemästet' u. s. w. (Kluge Wb. 218). Hängen nun diese germ. Wörter, wie Kluge vermutet, mit g. mats, an. matr, ahd. muos, as. mós u. s. w. zusammen (s. Fick III 229. Kluge Wb. 233 unter $m u s$ ), so hat man eine idg. Wz. $m a_{x} d$ - anzunehmen und idg. $m a_{x} z d h-m a_{x} z d$ sind aus idg. * $m a_{x} d-d h$ resp. *mad-dentstanden; s. $\bar{e} d h$ - repräsentiert somit ein idg. *inddh-1). Ob die nun gewonnene ursprüngliche Wz. $m a_{x} d$ - 'fett sein' im Grund mit s. mad- in mádati, mandati 'sich freuen, fröhlich, heiter sein, schwelgen in, sich gutlich thun in, sich an etwas berauschen; wallen, kochen' u. s. w. zusammenhängt oder nicht, ist nicht zu entscheiden. Die Bedeutungen lassen sich wohl ohne allzu grosse Schwierigkeit vermitteln.

Die Wz. dürfte als měd- (mø̆d-, mad-, mod-) anzusetzen sein (s. Fick I4 507. BB. I 63). mēd in $\mu \eta \dot{\delta \in \alpha ; ~ m e d-~ i n ~} \mu \in-$

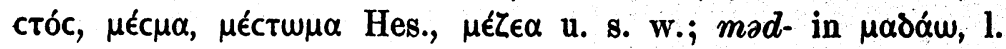

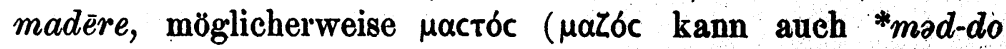

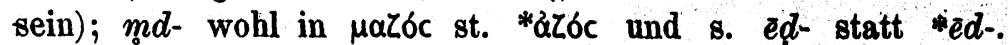
Diese Wz. ist von měd- 'messen' zu trennen, wozu Schade 597, Osthoff Perf. 71, Noreen Urg. judl. 33, Wadstein Nord.

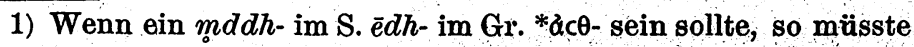
ein idg. mødd- im S. éd- im Gr. ${ }^{*} a c \delta-(* d Z-)$ sein. Ich vermute, dass wir beide Formationen entdecken können. $* a c \theta$ - erkenne ich in $z^{z} c \theta-\lambda o ́ c$, nur dass der Vokal nach einst daneben liegende Formen mit ${ }_{\mu} \mu c \theta-$ umgebildet worden ist. In ungekehrter Richtung ist die Ausgleichung vorgegangen bei mdd-. Daraus würde gr. *az-,

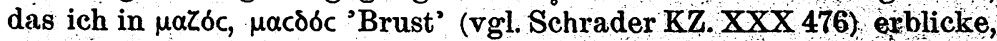
nur dass * $\alpha \zeta$ - durch daneben liegende Formen mit $\mu \in \zeta-\mu$ - bekommen

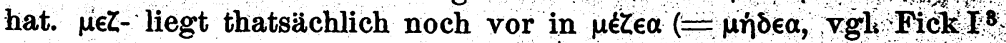
170. 390 . II 183 u. s. w. ${ }^{4}$ 507). mdd-findet sich möglicherweise in s. èda-, ëda-ka- 'Fettschwanzschat' (Bhāvapr. 2, 10. Pān. IV I, 4 G), unsicher wegen êtaka- Kāuç. S. 41, 6. Nur ist hier das $d$ befremdend, kann aber wohl als sekundär gefasst werden (vgl. y. Bradke ZDMG. XL 681). 
Tidskr. f. fil. $\mathrm{X} 227$ f. u. A. isl. matr, g. mats, d. mus gezogen haben (noch andre ziehen g. mats zu ahd. (stein-)mezzo u. s. w.). Die mir. Wortsippe meth (gen. meith) 'fett', methciim 'mäste', methe 'obesitas' gehört auch hierher, obwohl die Konsonantverhältnisse Schwierigkeit machen; wohl $=$ mezd-.

v. Bradke ist geneigt eine Wz. meid- (s. mēd-, mid-, mind-). überhaupt zu leugnen: s. médin- 'Genosse, Liebhaber, Verbündeter' lässt sich gewiss auf die hier behandelte Sippe beziehen, entweder mit der von v. Bradke angenommenen Bedeutungsentwickelung oder mit Annahme einer Bedeutungsentwickelung etwa wie in isl. motunautr ('Teilnehmer derselben Kost). Aber um die von Grammatikern und Lexikographen aufgestellte Wz.' $m i(n) d$ - kommt man nicht so leicht herum. Besonders schwerwiegend sind in dieser Hinsicht die von Geldner KZ. XXVII 249 (vgl. Bartholomae ZDMG. XXXVIII 132. Ar. F. II 130) zweifelsohne richtig erklärten avestischen Wörter mōistra oder myastra, mōidãstvâm, u. s. w., die auf eine W\%. meid- zurückweisen. Vielleicht gehört auch air. mīad 'Ehre' hierher und erweist auch seinerseits eine Wz. meid-. - Über mèdin- noch anders Weber KSB. I 400.

20. Skr. yéşăati und Verwandtes.

S. yểăati bedeutet 'wallen, sprudeln, rollen'; niṣ̌yesṣ̆ati 'herausquellen, ausschwitzen' ist nach Fick I 402 mit yásyati, yasati, yayasti 'sichs heiss werden lassen' u. s. w. zusammenzustellen. Die formelle Erklärung ist folgendermassen $z u$ fassen: es ist eine reduplizierte Form, der Bildung nach von derselben Art wie s. sáścati aus *se-sqe-ti, wovon etymologisch wohl zu trennen sind: $a-s a-s ̌ c a ́ n t-, a ́ s a-s ̌ c a n t-(i n a ́ s a s ̌ c a n t \bar{\imath} \mathrm{N}$. Sg. F. und D. F.), á-sa-šc-uṣ̄ ' unvergleichliche Fülle habend; F. Pl. die in reichlicher Fülle fliessenden Ströme’, wozu nach W.

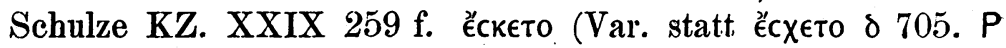
696) aus *séske-to, mit á-sak-ra 'nicht versiegend'; ja-kṣ̆-ati 'essen' von ghas- (ághas, ákșan u. s. w.), ca-kșsa-ti, - $\bar{e}, \quad s a-$ $j j a-t i,-e$ aus * se-zge-ti (Osthoff Perf. $31 \mathrm{ff}$. ), vgl. unthematische Bildungen wie sá-śc-ati (3 Pl., bá-bhas-ti $3 \mathrm{Pl}$. bápsati, ca-

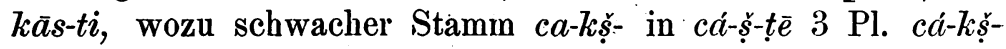
$a t \bar{e}, j a-k s \underline{s}-i-t i$ 'essen', já-kṣ̆-at- 'lachend' von has- (hasati u. s. w.), ja-jan-ti, da-dhan-ti, ma-mat-ti u. s. w. (vgl. Whitney $\S \S 640.645 .673 .675 .677 .679)$; gr. $\mu \epsilon \in \beta \lambda \epsilon \tau \alpha$, Aor. (Impf.), 


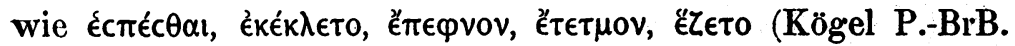
VII 192 Anm.; G. Meyer § 529, Schulze KZ. XXIX 249 N.), wovon ein sogen. Plusquamperf. wie acakrat nicht wesentlich verschieden ist. Es ist entstanden aus ar. ${ }^{*} i a-i s ̧ a-t i$, idg. *ie-ise-ti. Ein andrer Fall von derartiger Präsensbildung mit Reduplikation ist $l a$-șati 'begehren' aus *la-lsa-ti von der Wz. las- in lasati 'strahlen, prangen, erscheinen; lustig sein, sich vergnügen', gr. $\lambda_{l} \lambda \alpha i^{\prime} \mu_{\alpha} \alpha$ aus $* \lambda_{l}-\lambda \alpha c-j o-\mu \alpha l$ u. s. w.

21. Skr. mlēechá- und Verwandtes.

Altindisch mlēcchati 'wälschen, eine Sprache kauderwälschen', mlēcchá- 'Fremder, Barbar', wozu mlēcchata F. Abstr., mlēcchana- N. 'das Kauderwälschen', mlēcchitaka-, Inf. mlēcchitaväi, unbelegte Formen wie das Part. mlissta- und andres mehr (vgl. Whitney Wurz. 128), sind trotz mehreren Versuchen noch nicht etymologisch erklärt.

Pott Wurzelwb. III ${ }^{2} 362$ stellte in unrichtiger Weise die genannten Wörter zu d. wallschen, aber unter Annahme der ohne Zweifel etwas Wahres enthaltenden Ansicht Pictets Orig. indoeur. 56, dass abg. mlzcati verwandt sei.

Havet MSL. VI 238 erklärt mlēcchá- aus *mlai-s-qound stellt es, abgeseben vom Suffix, dem gr. $\beta \lambda \alpha ı c o ́ c$ gleich, das er aus *mlai-s-uo- (er vergleicht 1. fuscus : furvus) deutet; $\beta \lambda \alpha$ cóc bedeutet 'windend, stammelnd', was sehr gut $\mathrm{zu}$ der Bedeutung von mlēccha- passt. Nichts destoweniger ist diese Zusammenstellung aufzugeben und zwar aus folgenden Grtinden. Zunächst ist die Etymologie von Bugge KZ. XIX 433, wonach $\beta \lambda \alpha u c(c) b ́ c$ zu an. kleiss 'lispelnd' gehören soll, an sich \$o einleuchtend, dass sie als gesichert gelten darf (vgl. Verf. P.-BrB. XIV 321) ${ }^{1}$ ). Ausserdem aber kann mlêcchá- nicht von einer bestimmten Gruppe andrer sanskritischer und prakritischer Wörter getrennt werden, die unmöglich mit $\beta \lambda \alpha_{1}$ cóc (l. bloesus) zusammengehören können:

Schon vor Havet hatte Bloomfield AJoPh. VI 46 ff. eine andre Zusammenstellung ausführlich zu begrunden gesucht. Er stellt das Wort zum griech. Stamm $\alpha \mu \beta \lambda \alpha \kappa o-(\alpha \mu \pi \lambda \alpha \kappa o-)$ im

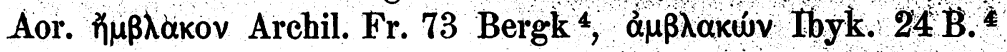

1) Vgl. Fröhde BB. I 204, der gleich Bugge auch 1. blaesus als urverwandt ansieht; während sowohl Fröhde selbst BB. I 332 als Weise Gr. Lehnw. 28 und Bersu Gutt. 130 es als Lehnwort ans dem Griechischen erklären. 
(wozu Präs. ả $\mu \beta \lambda \alpha \kappa i c k w$ Phintys bei Stob. Flor. 74, 61. Theages bei Stob. Flor. 1, 67. 68 nach Meineke) ${ }^{1}$ ), und nimmt eine Wurzelform mlek- (melk-), d. h. wohl melq- (mleq-), an. Skr. mlēcchati liess er aus *mle $(k)$ sketi oder ar. ${ }^{*} m l a^{i}(k)$ skati entstanden sein. Diese Erklärung ist in lautlicher Hinsicht unmöglich. Dass aber trotzdem Wwzelverwandtschaft anzunehmen ist, glaube auch ich.

In andrer Weise haben folgende Forscher die Frage zu erledigen gesucht. E. Kuhn KZ. XXV 327 erklärt sich das Verhältnis zwischen s. mlēcchá- und pāli milakkha- (Beitr. \%. Pāligr. 28) so, dass dieses aus idg. *mlosqo- entstanden, jenes dem Verbum mlēcchati aus *mlesceti nachgebildet sei, indem $\bar{e}$ aus idg. $\breve{e}$ in der von J. Schmidt KZ. XXV $60 \mathrm{f}$. (vgl. E. Müller Pāli lang. 25) dargelegten Weise entwickelt sein soll. Danach bespricht sie auch J. Schmidt KZ. XXVII 333, ohne sich über den Ursprung des $\bar{e}$ in mlēcchá- zu äussern. Dass es in der von E. Kuhn gedachten Weise entstehen könne, wird wohl niemand mehr behaupten. Bartholomae Stud. z. idg. Sprachgesch. I 45 weiss ebenfalls das $\bar{e}$ nicht zu erklären, ausser durch Annahme einer Wurzel auf ai ; Zubatý KZ. XXXI 14 nennt mlēcchati dunkel. Ich werde jetzt den Versuch machen in Zusammenhang mit der etymologischen Deutung das $\bar{e} z u$ erledigen.

Wir haben gewiss von der bei Fick I 175. 721. III 235 erörterten Wurzelgruppe, die ein einfaches Element mele- 'reiben, zermalmen, mahlen' u. s. w. aufweist, auszug'ehen. Über die aus diesen Bedeutungen eventuell sich entwickelnden Bedleutungen wie einerseits 'schwach, schlaff, lässig werden', andrerseits 'weich, sanft, mild werden' vgl. Verf. P.-BrB. XV 227 und die daselbst zit. Litt. Daraus sind mit verschiedenen Wurzeldeterminativen verschiedene erweiterte Wurzeln entstanden z. B. mel $(e)-d$ - in $\alpha \mu \alpha \lambda \delta u ́ v w, \beta \lambda \alpha \delta \alpha \rho o ́ c$, s. mardati, mradate $\bar{e}$, l. mollis u. s. w. 2. $\operatorname{mel}(e)-q-$, 3. $m e l(e)-d h$-. Von diesen interessieren uns hier zunächst die beiden letzten Varianten.

Die Form mel(e)- $q$ - erscheint als thematischer Verbalstamm mit schwacher Wurzelformation in $\eta \mu \beta \lambda \alpha \kappa o v$ aus (a)mlq-

1) Es wird hier von den Formen mit $\pi: \not \mu \pi \lambda \lambda \alpha \kappa i c \kappa \omega$ und $\eta_{\mu} \mu \pi \lambda \alpha-$ kov abgesehen, die schwer zu erklären sind; vielleicht ist $\pi$ aus $\beta$ durch Assimilation an $\kappa$ in bezug auf die Artikulationsart entstanden. 
(vgl. Fick BB. V 168), weiterhin in $\beta \lambda \bar{\alpha} \xi, \beta \lambda \bar{\alpha} \kappa o ́ c$ (vgl. $\beta \lambda \eta-$

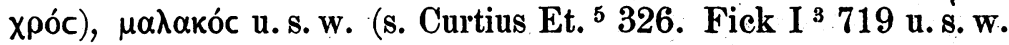
GGA. $18811450 \mathrm{f}$. Bloomfield a. a. 0.); weiterhin abg. mlzcati 'schweigen' aus dem Aor.-St. mlq $\bar{e}-\left({ }^{*} m l z k \bar{e}-t i\right.$ - wie g. fahēdi-u. s. w.). Hierzu kann, wie schon bemerkt, s. mlēcchánicht gezogen werden.

Die 'Wurzel' mel(e)-dh- erscheint in s. márdhati 'im Stiche lassen, vernachlässigen, nachlassen, aufhören, lässig werden', mrdhrá- 'Schmähung', $\mu \alpha \dot{\lambda} \lambda \theta \eta, \mu \alpha \lambda \theta \alpha$ kóc, air. mellach, 'A $\mu \alpha \alpha \lambda \theta \epsilon € \alpha$. Hier erst kann der Versuch gemacht werden das ai. mlecchá- u. s. w. einzuordnen. Und zwar können wir folgende Wörter vereinigen 1. mürchati, mürkhá- 'stumpfsinnig', prākr. mucchà 'Ohnmacht', mucchiya- (=s. mürchita-) 'verliebt, gierig', mukkha- 'töricht'; 2. s. mlēcchati, mlèccháund pāli milakkha-; prākr. miliccho ist vielleicht doch anch eine konsequent entwickelte Prākritform, nicht eine prākritisierte Sanskritform, wie angenommen worden ist. Dass wir hier in den Verben Inchoativbildungen auf $s q h \bar{o}$ vor uns haben, darf nach den Untersuchungen Zubatýs KZ. XXXI 14 ff. als wahrscheinlich angesehen werden. Demnach ist mürchati aus *ml̄dh-sqhe-ti entstanden. Ob mürkhá- etwa ein *ml̆dh-sqhooder eine prākritische Bildung, etwa ${ }^{*} m \bar{l} d h$-sqo-1), wie $\mathrm{Zu}$ batý a. a. 0.15 annimmt repräsentiert, ist nicht mit Sicherheit zu bestimmen ${ }^{2}$ ). So viel ich sehen kann, hindert nichts anzunehmen, dass mürkha- sich ebenso zu mürchati verhält wie mlëcchá, milakkha- zu mlëcchati. Wị mürchati mürkhd- ist nun mlēcchati mlēcchd-milakkha- gebildet; ist eigentlich damit identisch. Nur hat man von einer andern Form der Wurzel auszugehen, nämlich von mle-dh-. Es sollte eine idg. Bildung *mledh-sqhō- *mledh-sqheti im Sanskrit nur *mlakkha*mlacchati heissen. Eben daraus ist pal milakkha - wozu ein *milaccha- denkbar wäre - zu erklären. Vielleicht ist miliccha- mit dem yorauszusetzenden *milaccha- in irgend einer

1) Eine solche Grundform stellt freilich Zubaty nicht auf, es kommt hier nur auf das Suff. an.

2) Got. malsks ist *moldh-sq(h)o- mit anderm Wurzelablaut. Dagegen kann lett. mullis 'einfältiger Tropf' wohl nicht unmittelbar zu mürkha- gehören (Wiedemann BB. XIII 308), sondern ist wohl $=\mu \alpha \lambda \alpha k-\delta c$ (zum Wurzelablaut s. jetzt Bezzenberger BB. XVII 215). 
Weise zusammenzubringen, was ich nicht entscheide. Eine andre Möglichkeit wäre, folgende Entwickelung anzunehmen: *mledh-sqhā zu ar. *mladgha $\bar{a}$ und *mledh-sqhe- zu ar. ${ }^{*} m l a-$ $d j(h) a-$ zu *mlajj(h)a- (vgl. Bartholomae Ar. F. I 3 ff. II 54 ff. KZ. XXVII 351 ff. Stud. z. idg. Sprachgesch. I 3 ff. Brugmann Grundr. I \& 552, vgl. § 591; Osthoff Perf. 33 ff., vgl. Meringer ZföG. $1888141 \mathrm{ff}$.). Jedenfalls gewinnen wir dadurch kein ind. $\bar{e}$. Ich glaube, dies ist folgendermassen entstanden. Es gab neben der $-s q \bar{o}$-Bildung noch mehrere Formen, wo die Verbindung $m l e d h+$ Dental vorkam. Ein solches Beispiel ist namentlich part. ${ }^{*} m l e d h$-to- zu ar. ${ }^{*} m l a d ~ d h a-$ zu s. ${ }^{*} m l e ̈ d h a-$. Es mag noch viele andre Formen, z. B. in einem unthematischen Verb, gegeben haben, wo die Voraussetzungen eben die selben waren. Ich finde es möglich und in anbetracht der Unmöglichkeit der früheren Erklärungen, wahrscheinlich, dass $\bar{e}$ in mlēcchati, mlēcchá- eben von solchen, einst vorhandenen Formen tibernommen worden ist.

Freilich, an sich wäre es nicht unmöglich für s. mlēccháein idg. *mlai $s q(h)$ - anzusetzen. Man hätte dann an die sogen. Wurzel mlay- in s. mläyati (vgl. Verf. P.-BrB. XV 226 ff.) anzuknüpfen. Diese Wurzel ist natürlich sekundär mit $i$ vom Präsensstammsuffix hertubergenommen, $d . h$. aus urspr. *mläa-io Denn es ist evident, dass s. $m l a$ - 'welken' u. s. w. mit $\alpha \mu \alpha \lambda$-óc,

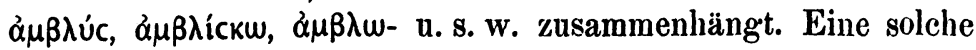
Annahme aber wird durch pāl. milakkha-, das von mlēcchati, $m l e \overline{c c h a ́}$ - nicht getrennt werden kann, hinfüllig.

22. Skr. adhra- 'arm, durftig, gering'.

Das Wort kommt an 4 Stellen des RV. vor I 31, 14. VII 18, 17. 41, 2, X 117, 2; meist scheint die Bedeutung 'schwach' zu passen, wie denn auch Ludwig an zwei Stellen tibersetzt. Das Wort ist noch nicht etymologisch gedeutet; denn der Vorschlag von BR. und Grassmann, dass es gleich $\bar{a}+d h r_{-}-$sei, gibt keine greifbare Bedeutung. Auch sehe ich innerhalb der indischen Sprachen keine Wortgruppe wozu $\bar{a} d h r a^{-}$- ungezwungen gezogen werden könnte ${ }^{1}$ ). Es gehört,

1) Es sei denn ádhara-, adhamá-, vgl. Bartholomae AF. III 5 f. 32 , der auch av. ādrēng y. 29,3 mit der Übersetzung 'die Untergebenen' dazu stellt. 
wie es scheint, mit zum ältesten indischen Wortvorrat, weshalb sein indogermanischer Ursprung noch unabweislicher erscheint. Ich stelle nun $a d h r a ́-$ direkt zu gr. vwӨpóc. Jenes steht für *ādhró- mit vom Akzent bedingter schwacher Wurzelstufe. Das idg. Paradigma war etwa nódhro-: ñ. $d h r e ́-$, woraus die indischen und griechischen Formen leicht erklärbar sind. Die Bedeutungen 'schwach' von adhrá- und 'faul,

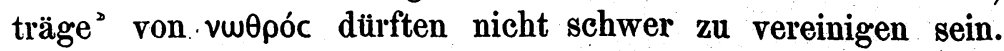
Die eine Eigenschaft wird sich leicht als Folge der andern

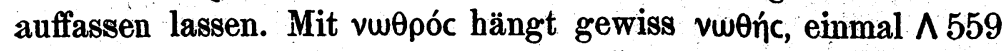
von Homer verwendet, als Beiwort zu ơvoc, zusammen. Von den landläufigen Etymologien z. B. Döderleins Gloss. N. 232 von

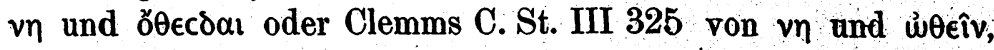
ist keine annehmbar, am wenigsten, wenn de Saussure in seiner Heranziehung von vó $\theta$ oc Recht haben sollte (Mém. 156).

23. Skr. máyas N. 'Labsal, Lust, Freude'.

Das Wort kommt im RV. zièmlich oft vor, aber nur im Nom. und Akk. Sing. in Verbindung mit bhávati, krnotit, 'geben' (wie pruáti, ráti, dáști u. s. w.) und dadhé ('empfangen'). Es ist mir wahrscheinlich, dass dies Wort durch eine Wurzel mei- 'mild, gelind, freundlich sein' mit s. mitrdM. N., dem einzigen Nomen zur Wz. mid- (im Falle mitrá- mit de Saussure MSL. VI 246 ff. aus *midtlo- zu erklären ist) zusammenhängt. Ich ziehe auch hierzu l. mitis 'mild, gelind, freundlich', air. moith (Stokes KSB. V 18). Es repräsentiert wohl eine Bildung mej-ti- aus einer jo-Bildung, die im Nom. auf $-i-s$ ausging. Ob die Wurzel mei- etwa mit smei'lächeln' identisch ist, lässt sich nicht sicher behaupten ${ }^{1}$ ).

\section{Skr. ?’şvá- 'hoch, emporragend'.}

Es mag richtig sein, was mehrere, z: B. Grassmann s. v.; annehmen, dass rớvá, av. ereshva- (wozu ereshi 'das Aufrechtstehen, die Gradheit, Wirkliehkeit, Wahrheit', s. $?^{\prime}-i$; vgl. Th. Baunack St. I 393. Bartholomae A. F. IH 32) mit einer durch $s$ erweiterten Wurzel, die bei o $\rho$ - in $\delta \rho-v u-\mu$ r vorliegt, zusammenhängt. Hier will ich nur auf ein andres Wort hinweisen, das meiner Ansicht nach im Nominalstamm das- 


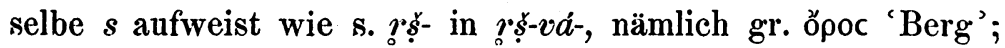
uber dessen Verwandte s. Verf. KZ. XXX 419 f. Man kann rşvvá- als eine Sekundärbildung ansehen wie arnavá- u. s. w. (Whitney $\S 1228$ ). Aber nichts hindert auch, eine Primärbildung in ihm zu erblicken, die von einem, mit dem Nominalstamm öpoc identischen Verbalstamm ausgeht, vgl. ürdhvá(Whitney § 1190).

\section{2ō. Skr. ganda- 'Wange' und 'Knoten'.}

Fick I 69 trennt diese Wörter und stellt ganda- 'Wange' zu rvá $\theta$ oc 'Kinnbacke', lit. źándas 'Kinnbacke' (vgl. J. Schmidt Vok. I 153. KZ. XXV 125 N. 2), gança- 'Knoten'

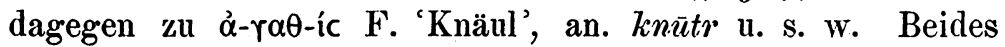
ist in lautlicher Hinsicht unannehmbar. Ich vermute, dass ganda- 'Wange' und ganda- 'Knoten' dasselbe Wort sind, wie auch BR. und Boehtl. annehmen. Nach Caraka I 28 auch 'Kropf und andre Halsanschwellungen'. Und zwar ist in beiden Fällen die Grundbedeutung 'etwas rundes, kugelförmiges'. Ich deute ganda- aus *gal-n-da- und stelle es zu l. gland(Nom. glans), abg. želadb (vgl. J. Schmidt Vok. II 357) mit

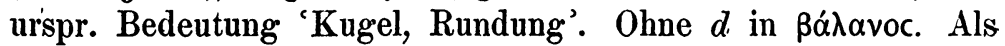
Wurzel hat man ein gele- oder dgl. zu statuieren. Diese einfache Wurzelform erscheint in lit. gilë aus *galēe, wie auch in $\beta \hat{\omega}-$ $\lambda \circ c$, s. glä-u-, sowie in Weiterbildungen wie 1. glēba, globus, ahd. cholbo, an. kōlfr, l. glomus u. s. w. Mit d-Ableitnng s. guça- 'Kugel', wohl aus *galdo-, an. klōt, 1. glădius Wr.glè-d-, gla-d-) u. a. Wörter (vergl. Fröhde B13. X 298. Verf. P.BrB. XIV 307 N. 1), mit $t$-Abl. s. gutika 'Kugel', abg. glota 'turba'. Gewiss gehört auch s. jōgla- 'Kinn' hierher. In bezug auf den Vokalismus verhält sich jōda- zu guda- wie khọ̄a- (khō$t a$-) $\mathrm{zu}$ einem vorauszusetzenden *khuda- (vgl. g. halts, sphotazu sphutati u. s. w. (s. Fortunatov BB. VI 216. Verf. De deriv. vb. contr. 128 N. 2). Zu ganda- noch gandu- 'Kopfkissen' und gandū 'Knoten, Knolle, Gelenk'. Zur Nasalierung: ganda- u. s. w.: $g(a) l(a) d$ - vgl. ahd. ags. as. springan, abg.

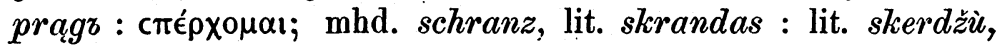
skardýti; s. kñ-n-táti, ahd. scrintan, lit. skrentù : lit. kertù, abg. črbta, s. kartá-; abg. kręt-aja 'flecto', krątz 'tortus', s. krnátti 'dreht, spinnt' : crtáti 'knüpft'; s. vronákti 'dreht',

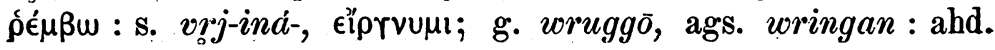


wurgen, lit. veržiu 'schnüre zusammen'; s. trọát-ti, lit. tréndu:

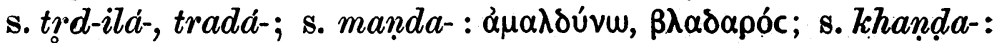

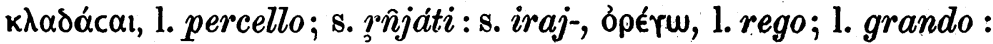
abg. gradt; ; mhd. schrimpfe : aisl. skorpna; abg. prędati 'springen', lit. sprendžu 'spanne', an. spretta: d. spratzen; abg. glę-

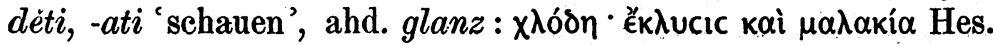
u. s. $\left.\mathbf{w}^{1}\right)$.

\section{Skr. sphulirgar 'Funke'.}

Soviel ich weiss, ist dies Wort, ved. višpulingaká- 'Funken sprühend' RV. I 19112 von vişphuiliraga- 'Funke' Çat. Br. u. s. w., noch nicht etymologisch gedeutet worden. Man könnte daran denken, es zu den unter '2 sparg' bei Fick I 253 verzeichneten Wörtern zu ziehen, s. sphürjati 'donnern, rauschen' u. s. w. ${ }^{2}$ ). Dem stelle ich den folgenden etymologischen Versuch gegentiber. Wie d. funke von einer Lichterscheinung seinen Namen hat, so ist das. wohl auch bei sphulingader Fall gewesen. In bezug auf die Form scheint -ga- eine $\mathbf{A b}$ leitung zu sein wie in s. sgrnga- u. s.w. (s. Verf. Beitr. z. grieeh. Sprachkunde 9 ff.). Dass aber eine solche Erweiterung schon idg. den Charakter eines Wurzelelementes angenommen haben kann, ist selbstverständlich und wird in vorliegenden Falle durch meine Zusammenstellung wahrscheinlich. Ich deute nämlich sphulinga- aus (s)phalan-g- und stelle es zu nhd. fink aus ndd., ndl. fink 'hurtig, behende', alt. nhd. finken 'flimmern, glänzen', nhd. funkern 'flimmern', Wörtern, die gewiss altererbt sind. Auch 1. splendeo mit Ableitungen und lit. splendžu splendeti 'glänzen' scheinen hierher zu gehören. Sie können wohl aus sphleng-d-gedeutet werden, aber nichts hindert eine g-lose Form splen-d- anzunehmen. Dann könnte

1) Wohl auch s. pinda-, pindtiá-, ags. flint : s. pidaka-, pitaka-, vgl. 1. pila (Froehde BB. X 298; anders Osthoff KZ. XXIII 85 f.). Vgl. noch lit. sklandau 'schwebe, schwanke's g. halts, s. khōda-

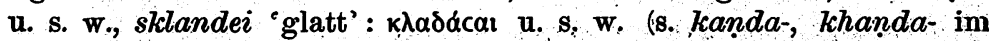
Text), s, chrnatti, chrnttē : an. skarta, lit, galándu, glándau (vgl, Bezzenberger ZGLS. 68 N. 3 f. BB. V 168. Verf. KZ. XXX 441 Anm.2), s. abhivlangá- (vgl, Geldner Ved. St. I 140, Oldenberg GGA. 1890 412): abhivlágya-.

2) So jetzt, Fick folgend, auch Persson Wurzelerweiterung u. Wurzelvariation $17 \mathrm{f}$. 


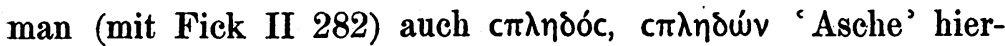
her ziehen und wohl aus sphlñ d- erklären. Die $n$-lose Form idg. (s)pha $a_{x} l-g-\left(:(s) p h\left(a_{x}\right) l-n-g-=a n\right.$. barki : $\varphi \alpha \alpha \alpha-\gamma-\gamma-$

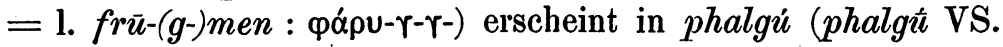
$24,4)$ 'schimmernd, rötlich schimmernd; winzig, schwächlich', phalgúa- 'gering, schwächlich' RV. IV 5, 14, lett. spulguit 'glänzen'. Möglicherweise sind in phalgú-, phalgúa- zwei Wörter zusammengeflossen: 1. idg. (s)phaxl-g-u- 'schimmernd', 2. idg. (s) $\left.p h a_{x} r-g-u-{ }^{1}\right)$ 'winzig' von einer Wz. $s p(h) a_{x} r a_{x}-g^{-}$'platzen, bersten' in s. sphúrjati mit mannigfachen Bedeutungen (von denen einige z. B. 'flammen' möglicherweise sphürjáyan RV. X 87, 11 wohl zu 1 gehören), lett. sprēgstu 'platze, berste’, sprāgstu id., lit. sprógstu 'prassele, spriesse’ u. s. w.

27. Skr. jihreeti und Verwandtes.

S. jihrēti mit der Bed. 'sich schämen' findet sich in der Form jihryat zuerst in MS. Sonst begegnen schon im RV. a-hrayana-, das mit 'keck, kühn, üppig' uibersetzt wird; ferner a-hraya- und a-hri-, wozu a-hri- Cat. Br. 11, 3, 3, 5. Übrigens begegnen hiermit zusammenhängende Wörter meist in den Brāhmana und der späteren Sprache. Es gentugt aber erwähnt zu haben, dass die Wortsippe schon rigvedisch ist.

Soviel ich weiss, ist für diese Sippe noch keine annehmbare Etymologie gegeben ${ }^{2}$ ). Ich mache hier einen Versuch. Das deutsche 'sich schämen' hat sich anerkanntermassen aus einer Vorstellung 'sich bedecken' entwickelt, wie sich durch Vergleichung der ganzen Wortsippe mit Wurzel (s)kem(e)ergibt (s. z. B. Kluge Wb. u. scham. Verf. KZ. XXX 428 ff.). Ebenso ist es mir wahrscheinlich, dass die idg. Wurzel ghrei-, oder wie sie aufzustellen ist, 'bedecken' oder 'sich bedecken" bedeutete. Dann gewinnen wir eine Anknüpfung an eine germanische Wortsippe. Am klarsten tritt diese Bedeutung hervor in an. grīma F. 'Maske', ags. grīma, grïmma M. 'masca, larva'. Besonders beweisend sind die mannigfachen Bedeutungen des an. gri-ma nach Fritzner ${ }^{2} 1$. 'Schirm, der das Gesicht verbirgt', 2. 'eine Art von Panzer, der das Haupt

1) Vgl. jetzt Fick I ${ }^{4} 149$ f. mit 337 f. 573 . Über phalgúa'nichtig' jetzt anders Hoffmann BB. XVIII 154.

2) Sonne KZ. X 101 (vgl. J. Schmidt Vok. II 255) zieht es zu Wz. ghar- 'glühen', was mir sehr zweifelhaft scheint. 
und den Hals des Kriegspferdes bis zum Sattel deckt', 3. 'Saturnsbild', 4. 'Nacht', 5. 'dunkle rätselhafte Rede'.

Diese Wörter haben wahrscheinlich nichts mit an. grī-na, ahd. grinnan, nhd. greinen (wie Fick III 111 u. a.) zu thun, weil die Bedentungen zu fern liegen.

28. Skr. ar šs 'stechen, beschädigen'.

Ein Verb *? ${ }^{*}$ yati ist freilich nicht belegt; dass es aber vorhanden gewesen sein kann, beweist das gir. ápócccw, das meiner Meinung nach zur s. Wz. poś gehört. Das gr. Verb bedeutet 'schlagen, klopfen, stossen' (z. B. $€$ 248. 426. N 577. M 384 u. s. w.). Dass die Schallbedeutung primär sei, ist durch nichts zu beweisen. Die appoximative Allgemeinbedeu. tung kann gar wohl als 'stossen, stechen, stecken' angesetzt werden. Diese Bedeutung nun erscheint in den sanskritischen Wörtern. Die betreffenden sind aršasaná- 'stossend, stechend, verletzend', anarša-räti, 'der Gaben hat, die nicht verletzen', An-aršani- 'Eigenname eines von Indra bekämpften Dämons'; dazu wohl auch an-rgkşarara- 'dornenlos' in der vedischen Litteratur. Da die Wz. ar-k- eine Erweiterung der einfachen Wz. ar-1) ist, erklärt sich leicht das Verhältnis der Wz. riś- (idg. re $i \hat{k}-)$ zu ark-: $\hat{k}$ ist als Erweiterung angetreten, sowohl an einfache Wz. ar- als auch an das durch $i$-Erweiterung entstandene (a)r-ei-. Daher der Parallelismus ark- $:$ reik-; $i$ kann in reikk- als Infix gelten, nur hat man sich zu vergegenwärtigen, dass die Infixerscheinungen nur durch Suffigierung erklärbar sind (Verf. De deriv. vb. contr. 109 ff. P.-BrB. XIV $315 \mathrm{fr}$. Noreen Urgerm. judlära 46, vgl. Bartholomae A. F. III 33 f.).

Ganz wie $a r-k-$ zu rei-k- (s. ros-: ris-) so verhalt sich ar-s- zu rei-s- (s. roş- : $\left.r i-s_{-}^{-}\right)$. Die erste Wurzel erscheint unter der Bed. 'stossen' im s. ro ợati (ud-, ni-; einfach rsşáti AV. IX $4,17)$, av. areshyant, Wz. rei-s- in s. reșatati 'verletzen', av. irishiñti (3 Pl.), raēsha (Jackson $\mathrm{AJ}$ oPh. XI 87 f.).

\section{Ap. ras- 'kommen'.}

Diese Wurzel kommt vor in den Formen aras-am Bh. I 54. II 28. 48. 63; parä-ras-am Bh. II 65, parä-ras-a (3 Sg.) Bh. II 22. 32. 52. III 3. 4; ni-ras-ä-tij (3 Sg. Konj.) Bh. I 24 (s. Spiegel Ap. Keilinschr. ${ }^{2}$ 238) und bedeutet 'kommen,

1) Eig. ara-k, woraus einerseits ark- andrexseits $r a k$ - in $\mathrm{s}$ rákłşas, av. rash̄ō, räshayainhēe (s. oben). 
gelangen zu'. Hiermit hat J. Darmesteter MSL. IV 225 f., wie mir scheint, evident richtig l. recens, der Part. eines Verb. *recere 'kommen', zusammengestellt; er vergleicht z. B. recens a vulnere 'qui vient d'être blessé', Verres cum e provincia recens esset, invidiaque et fama non recenti, sed vetere ac diuturna Cic. Verr. I 2, 5 'Verrès, retour de sa province' usw. Die bisher gewonnene Allgemeinbedeutung scheint 'aufkommen, von etwas kommen, emporsteigen' zu sein.

$\mathrm{Zu}$ dieser Bedeutung passt sehr gut eine germanische Wortsippe. Mhd. rëgen bedeutet 'sich erheben, emporragen', Faktitivum dazu mhd. regen 'etwas ragen machen, aufrichten, erregen, bewegen, wecken'. Dass die Wz. als rě̌k- anzusetzen ist beweist mhd. roche 'starr, steif'. Gehört ags. ofer-hrazian hierher (s. Kluge u. ragen), so muss $h$ - sekundär oder ein Präfix sein wie z. B. in g. h.ausjan u. s. w.

Formell könnte die s. Wz. raš- in raśand 'Strick, Wagenstrang, Zügel', raś-mán- 'Zügel' (a-raśmán-, stháa-rašman-), raš-mi- 'Wagenstrang, Zügel, Strahl', lit. riszti 'binden' (Leskien Ablaut 281), möglicherweise air. con-riug 'ligo' (Windisch KZ. XXIII 213) u. s. w. hierher gehören. Ich wüsste jedoch nicht die Bedeutungen zu vermitteln.

\section{Skr. vĩdu- 'fest' u. s. w.}

Der Verbalstamm vĩdaya- Kausat. und Part. vĩudita- kommen an 9 Stellen des RV. vor in der Bed. 'stark, fest machen', Med. 'sich stark erweisen, stark sein', Part. 'stark, fest' (wie

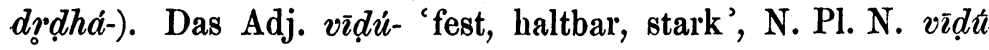
RV. I 39, 2 (Lanman Nouninfl. 415), N. Sg. N. als Pl. vidú RV. VIII 60, 9, und substantiviert vīlú mit der Bed. 'die festen Burgen' RV. I 71, 2. VIII 40, 1 (J. Schmidt Pluralb. 278 f.). Wie diese Sippe etymologisch zu deuten sei, ist schwierig zu bestimmen, und das folgende mag nur als ein unsicherer Versuch betrachtet werden.

Nach 0. Meyer Quaestiones Homer. $10 \mathrm{f}$. wäre vīlni- mit

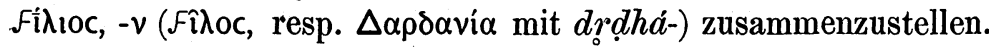
Nichts kann unsicherer sein. Entweder sind die griechischen Wörter echt griechisch; dann aber kann $\lambda$ und $d(l)$ nicht gleichgesetzt werden (eben so wenig wie anderseits $-\delta$ - in $\Delta \alpha \rho \delta \alpha v^{\prime} \alpha$ mit $\underline{d h} ! h$ in $d r d h a^{-}$); oder sie sind ungriechisch. Als indogermanisch können sie höchstens in Beziehung zu den 
iranischen oder thrakisch-phrygischen Familien gesetzt werden. Dann aber ist dem etymologischen Streben fast jeder Boden entzogen. Denn sei $-d(-l-)$ aus - $l d$ - oder $-z=d$ - entstanden: jedenfalls kann es keinem iranischen $l$ entsprechen; und wie der Reflex im Thrak.-Phryg. gewesen sei, ist unmöglich zu erraten.

Man könnte auch daran denken, $-\not-(-l-)$ aus intervokalischem -ž- zu erklären. Nach Bartholomae A. F. III 52 N. 1 vgl. I 20 N. 1 f. würde dies aber - $r$ - werden (vgl. Verf. BB. XV 178 N.1). Und auch wenn es an sich möglich wäre anzunehmen, dass unter gewissen Bedingungen auch $-d-(-l-)$ ein intervokalisches $-z$ - vertreten könnte, so ist dies doch bisher durch kein Beispiel erwiesen.

Wir müssen demnach andre Möglichkeiten in betracht ziehen. Das s. - $d-$ - kann aus $-z z d-$ (d. h. idg. $g d$ oder -r-; -r-; $-i$-zd-) oder aus ar. $-l d$ - (sei es aus idg. $-l d$ - oder aus ind. $-l d$ gewordenem idg. $-r d$-) erklärt werden. Dass aber das vedische $l$ aus - $l d$ - entstanden sei, ist, wenigstens bis auf weiteres, recht unsicher; möglicherweise ist jálhu - so zu erklären, vgl. Froehde BB. III $128 \mathrm{f}$. Fortunatov VI 217.

Aber auch wenn dem so wäre, sehe ich für die Annahme eines *ǔ̃l-d- keinen Anhalt. Und an ein *uั̌r-d- z. B. in vĩr-á-, l. vir u. s. w. zu denken ist um so weniger statthaft, als es noch nicht als völlig ausgemacht gelten kann, dass s. $-r d$ - in - $d$ - ubergehen kann.

Wir stehen demnach bei der einzigen Möglichkeit vīdaus *uzzz-d-zu erklären (vgl. Hübschmann KZ. XXIV 407 f.). Ganz einleuchtend wird dann die Annahme, dass diese 'Wurzel' aus einem einfacheren Element $u^{\text {z }} z$ - und dem Determinativ $d$ entstanden ist. Solcher Bildungen gibt es in den idg. Sprachen mehrere.

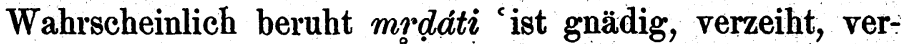
schont', mrdīk ká- N. 'Gnade', av. merezhdīka- 'Gnade' auf mrsśin s. mŕsşyate 'vergisst', marşáayate 'erträgt geduldig, verzeiht', lit. mir̃sz-ti 'vergessen' (Fick ${ }^{3}$ II 394. Hübschmann KZ. XXIII 386. XXIV 408. Brugmann Grundi. I \& 404 S. 301 u. A.).

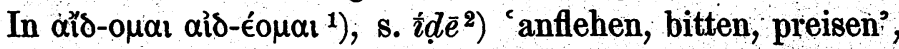

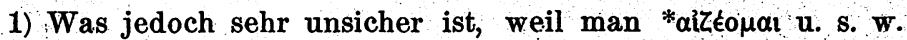
erwarten müsste. $\alpha i ́ \delta$ ćoul ist vielleicht aus $a u i-\alpha$ - von der Wz auei'wahrnehmen' gebildet (vgl. av. vaē-naiti 'sieht').

2) Eine andre Anknüpfung für $\dot{\imath} d \bar{e}$ jetzt bei Brugmann IF. I 
tạ- F. 'Verehrung, Preis' (ein deverbatives Nomen), g. aistan, l. aestimō (falls aus *aiz-d-itimo) u. s. w. (vgl. Bezzenberger BB. IV 313 f. Bechtel ebd. X 286. Bartholomae ebd. XII 91 u. A.) haben wir ein einfacheres Element aiss-, erhalten in s. $i \grave{s}^{-}, \bar{\imath} \grave{s}^{-}$(vgl. Bartholomae A. F. II 72 ff. Fick I ${ }^{4} 346$ ), ahd. èra 'Ehre', ags. äre 'Ehre, Erbarmen', an. öra, wahrscheinlich auch sab. aisos 'des Gebetes, sacri' (vielleicht Gen. eines St. aisu-), volsk. esaristrom 'sacrificium', umbr. esono 'sacer, divinus', osk. aisusis (N. Pl. eines Fem. auf $-i$, s. Bugge Ait.

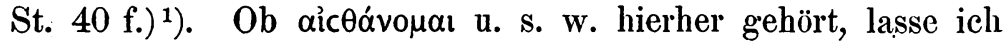
unentschieden (vgl. Bezzenberger a. a. O. Hoffmann BB. XV 62).

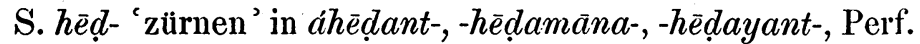
jiḥ̄̃la u. s. w., héda- hédas 'Zorn', av. zōizhdishta-, zōishnu(Jackson AJoPh. XII 68 f.) u. s. w. (dagegen wohl nicht jistayamnō, wie Geldner KZ. XXVII 243 f. annimmt), ahd. geist enthalten ein ghaiss-d-, ghis-d-, das eine Erweiterung mit $d$ von einem einfachen Element ghains, ghis- ist. Dies erscheint

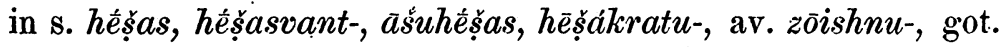
usgaisjan; usgeisnan, an. geisa 'wüten' (v. Bradke KZ. XXVIII 295 ff., vgl. Bechtel Sinnl. Wahrn. 104, jetzt auch Zubatý BB. XVII 326). Die approximative Allgemeinbedeutung der genannten Wörter dürfte 'heftig, hervorstürmen, rasen, wliten' sein (Pischel Ved. St. I $47 \mathrm{ff}$.). Ich kann nicht umhin die Wurzel $h \bar{e} s$ s. $^{-}$als eine $s$-Erweiterung der einfachen Wurzel ghei'treiben' in s. hinóti, äsuhéman- u. s. w. anzusehen.

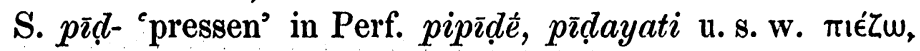

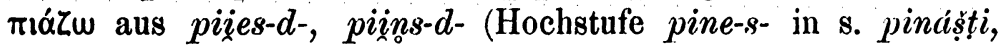
pratyapiğat, 1. pinso, pinsio; $\pi$ ríccw ist seiner Bildung nach noch unklar; s. Verf. De deriv. vb. contr. 109 N. 2) enthält ein einfacheres Element $p$ (i) ieses-, pis-; vgl. s. Perf. pipéșăa, Part. piș̌tá-, pináști u. s. w.

Möglicherweise haben wir mit v. Bradke KZ. XXVIII

171 f. (Ähnlich schon früher Bezzenberger GN. 1878 264). - Über die ved. Schreibungen îlē aber ídya- s. v. Bradke ZDMG. XL 668 N. 1, vgl. Roth KZ. XXVI $59 \mathrm{f}$.

1) Es mag hier beiläufig hervorgehoben werden, dass im gr. ífóc $\frac{c}{i} \rho o ́ c$ vielleicht zwei Wörter zusammengeworfen sind: eins $=\mathrm{s}$. ișirá-, eins aus *iss-ro- 'zu ehrend, göttlich, heilig', das vielleicht noch in s. isşira- 'Feuer' erscheint (vgl. Osthoff MU. IV 149 ff. Solmsen KZ. XXIX 349. Kretschmer KZ. XXXI 377. $421 \mathrm{~N}$. 1); die $r$-Ableitung noch in volsk. esar-istrom. 
298 N. 1 in s. mīl- 'die Augen schliessen' (in sammitlya RV. I 161, 2, mīlati u. s. w. B.) eine (dialektische) Entwickelung von $m \bar{\imath} l-m \bar{\imath} d$ - (vielleicht in mîdam 'leise') zu sehen ${ }^{1}$ ), und dies aus miž-d- zu erklären. $\mathrm{Zu}$ grund läge s. miș̆- 'die Augen aufschlagen', ni-mišáti 'die Augen schliessen, einschlummern'. Ein weiteres Beispiel des Wechsels $d: l$ ist s. $k \bar{u} d a y$ ati 'macht versengen' RV. ${ }^{1}$ AB. ${ }^{1}+:$ külayati S.,+ külita- C.

S. krídati 'spielen' ist m. E. (P.-BrB. XV 229) aus *krīž-dentstanden; dazu an. hrista 'schütteln'. Wurzelform ohne $-d$ in as. hris-jan, ags. hrys-jan, g. af-hrisjan 'abschütteln'.

S. krudáti ' untertauchen' Dhātup. 28, 100, 'dick werden' Mahīdh. zu VS. 25, 8, akrūdayat, krüdyamana-, cukrū dàyati Käth. 6, 3, kroōdá- AV. + 'Brust, Eber', av. khraozhdañt 'hart, fest', khraozhdva- 'hart', khruzhdra-id., l. crüdus zu s. kra-

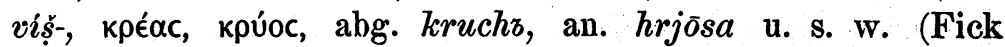
$\left.I^{4} 191\right)$.

Ich brauche kaum mehr Beispiele ${ }^{2}$ ) anzuführen, um das Nebeneinanderliegen von 'Wurzeln'. auf $s$ - und ebensolchen mit $d$-Erweiterung zu konstatieren. Wir sind demnach völlig berechtigt für vĩd- ein *ǔss-d-vorauszusetzen, wenn wir die Wurzelform utss- einzeln nachweisen können.

Wenn wir uns die Bedeutung 'stark, fest, kräftig' für vĩdúvergegenwärtigen, so liegt der Gedanke sehr nahe, dass $u^{7} s_{s}-$ (oder vielmehr $u \bar{\imath} s-$ ), mit $u \bar{\imath} s$ - in l. vīr-es, St. vīs-, identisch ist (s. J. Schmidt Pluralb. 384 f.). Wir haben hier eine nominalverbale Erweiterung mit s-Suffix von der Wurzel uễ $\left.j^{-3}\right)$. Als nominaler $s$-St. in s. váyas (idg. ueios-), l. vĩr-, als verbaler

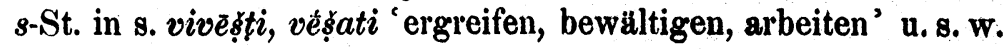

1) Vgl. noch z. B. ilā neben $i \grave{a}$, iḍā und sogar irā. Über diese Wörter vgl. vorläufig Geldner Stud. z. Av. I 64 ff. KZ. XXVIII 402. Th. Baunack Stud. I 374. 391 mit Bartholomae BB. VIII 213. 224 f. AF. I 21 N. 4. III 52. f. Verf. BB. XV 178 N. 1.

2) Man könnte daran denken s. vrîdyati 'sich schämen, aus *vriž- $d$ - zu erklären. Es kann jedoch eine dialektische (prākritische) Form für vrīd- sein. Man könnte es vielleicht mit g. wlits 'Angesicht', anda-uleizn (aus *ulīt-snó-), wlaitōn, an: līta 'spähen u. s. w. zusammenstellen. Es ist aber vielleicht besser es aus *ulaz- $d$ - zu erklären und darin einen s-St. der Wr. uel- (uer-) 'bedecken' zu suchen.

3) Diese Wurzel erscheint als Nominalstamm z. B. in 1. vi-m,

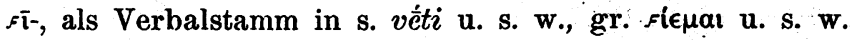

Indogermanische Forschungen II 1 u. 2. 
Wir begegnen hier der so überaus häufig bezeugten Thatsache, dass gewisse Verbal- (Temporal-, Modal-)Stämme mit ähnlichen Nominalstämmen parallel gehen. Ausiser den bei J. Schmidt a. a. O. erwähnten Fällen wie *bheios, ar. F. *bhiyās : bhyasin á-bhyasētam u. s. w., bhīs- in mnd. bister aus *bhiss-10-1);

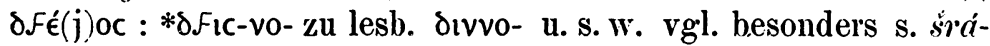

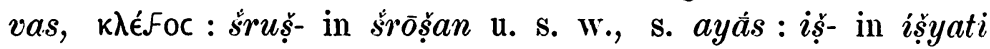

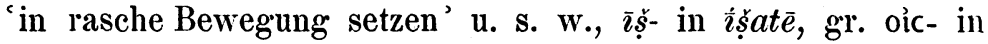
oîn u. s. w. Ich sehe hier von den Bedingungen des Ablautes $\bar{\imath}: \breve{u}, \bar{u}: \breve{u}$ ab.

Es steht somit der Annahme kein Hindernis entgegen,

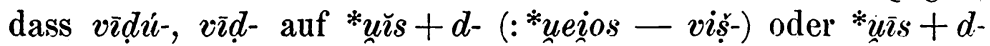
(: *uiiós - vïs̆s) zurückzuführen sei. Die Bedeutungen von vīd- und văšs- machen diese Zusammenstellung zu einer angemessenen. Dass in vĩdu-(pátmabhis RV. I 116,2) ein andres Wort zu sehen ist, das Bartholomae BB. VIII 224. XIII 87 zu av. vöizh-d-at (zu uik-) stellt, kann ich nicht glauben. $\mathrm{Zu}$ vĩd- zieht jetzt Jackson AJoPh. XII 67 f. av. vōishdat, voizhdayañt-. - Vgl. noch die Anknüpfung von Bloomfield AJoPh. III 3 .

\section{Skr. gup- 'beschützen'.}

Diese Wurzel kommt schon in RV. vor: Perf. jugupur VII 103, 9, Part. gupitá- zweimal, guptá-, gōpsyati AV. von einem gōpati, das nicht sicher steht, vgl. das Denom. gopāyáti im RV. Es ist allgemein angenommen, dass diese Wurzel im letzten Grunde aus gōpd́ 'Hirt, Behüter' entstanden ist. Nur möchte ich glauben, dass diese sekundäre Wurzel, falls sic wirklich auf diese Weise entstanden ist, nicht erst im Sanskrit (schon im RV.) entstanden ist, sondern schon in indogermanischer Zeit, d. h. dass gup- schon damals als Bedeutungszentrum Ableitungen zu grunde gelegt worden ist.

Ich stelle nämlich hier\%u nhd. koben, kofen, mhd. kobe 'Stall, Schweinestall, Käfig', mhd. kobel 'eng'es Haus', isl. kofe 'Hütte, Wetterdach, Verschlag, ags. cofa 'Gemach, Schlafgemach' (s. Kluge Wb. u. Koben u. Kobold). Ahd. chubisi 'Hütte' scheint auf einem alten $s$-Stamm, der jetzt verloren ist, $z u$ beruhen.

Auch im Griechischen gibt es wenigstens ein verwand-

1) Über die verschiedenen Erklärungen dieses Wortes s. Tamm Et. svensk ordbok 37. 


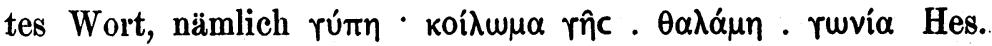
Darauf beziehen sich auch Teile der nächst vorhergehenden

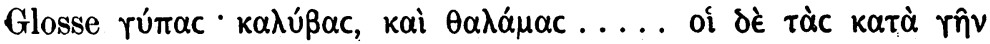

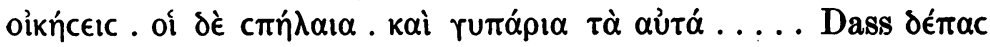
und andre Wörter bei Fick ${ }^{4}$ I 34. BB. XVI 286 hierher gehören, bleibt mir zweifelhaft (vgl. Verf. BB. XVIII 31).

32. Skr. ákș̌u-.

Das im RV. einmal vorkommende Wort ward fruher mit 'Netz' erklärt (s. BR. Grassmann s. v.). Von Boehtlingk Wb.

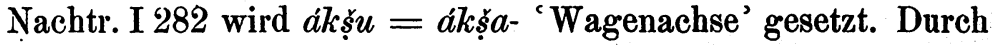
Vergleichung der Stellen AV. IX 3,8 und VIII 8, 18 wird von Geldner Ved. St. I 136 f. die Bedeutung 'Stange' gewonnen. Darnach wird das Wort RV. I 180, 5 gleich 'Stange des Wagens', d. h. 'Deichsel', erklärt. Die Richtigkeit dieser Bedeutung vorausgesetzt - und mir scheint sie sehr wahrscheinlich -, soll hier eine Etymologie vorgeschlagen werden.

Es ist wohlverburgte Thatsache, dass Wörter, die 'Stange' bedeuten, oft mit Wörtern mit der Bedeutung 'stechen' $u$. dgl. zusammenhängen. So hängt an. stika 'Stange', schwed. stake, d. staken mit stechen, an. stǫng, d. stange mit g. us-stiggan, an. stinga (Wurzel vielleicht $z$ dengh- s. oben, oder stengh- s. Fick

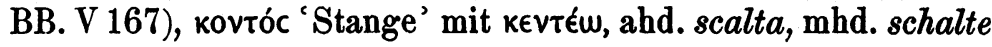
mit schalten (zu ahd. scëltan u. s. w.) zusammen (s. Verf. P.-BrB. XIV 313). Es ist demnach nicht unberechtigt in aksọ̆ueine Wurzel mit der Bed. 'stechen' zu sehen. Man könnte an $\not \not k$-, $\not k$ - 'scharf, spitzig sein' denken, und $b z u ́-c$ vergleichen. Es scheint mir jedoch, dass wir mit einer andern Anknupfung eine konkretere Bedeutungsentwicklung gewinnen konnen.

Es gab eine idg. Wurzel enegh- 'stechen', die als enghoder negh- auftritt $^{1}$ ). negh- erscheint in abg. nbza nbsti, pronoziti 'durchbohren', nozb 'Schwert', vévoxє, wohl auch vúccw (vgl. vukxácac vúzac Hes.) ${ }^{2}$ ). engh- widerum findet sich in

1) $\mathrm{Ob}$ diese Wurzel durch Nasalinfix aus ĕgh- 'stechen' (in Exxıc, Exîvoc, ahd. igil, lit. eźỹ s u. s. w.) entsțanden ist (wie z. B. Fick I ${ }^{4}$ 361. 501 vermutet), lasse ich dahingestellt.

2) Möglicherweise geht jedoch vúccu mit s. naghamāra-, naghārişa auf eine Wz. negh- zurück. Dass es neben negh- auch ein ne-i-ghgegeben hat, ergibt sich aus lit. nu-nižęs zu nižtù nižaú nižti 'krätzig werden', pa-nižtù 'anfangen zu jucken', néža (néžt) nëžéti 
Ërxoc 'Lanze' (vgl. Fick BB. I 341). Eine Ableitung auf - $u$ gab *éngh-su- : *ngh-s-éu-, *òngh-s-éu. Dies ergab eine Kontaminationsform *ågh-s-u-, s. ákșu-. Die Bildung auf $u$ ist völlig vergleichbar mit ỏzú-c aus dem $s$-St. ăkos-, ð̌kes-.

Mit den genannten Wörtern häng'en noch folgende $z u-$

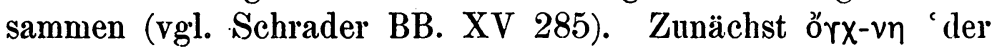

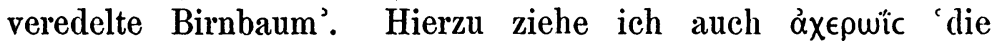
Weisspappel' aus nghér- und noch einem Element, das (wie schon Fick BB. XVI 171 bemerkt) wie ein Zusammensetzungsglied aussieht. -wic ist deutlicher $i$-St. und gehört $\mathrm{zu}$ lit. üsis 'Esche' und l. or-nus aus *öri-no- (Fick a. a. 0.). Vgl. hier-

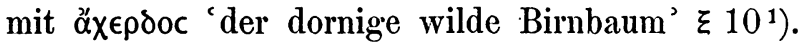

Es ist noch nicht ausgemacht, wozu ỏzún, das sowohl 'Lanze' als 'Buche' bedeutet, zu ziehen ist. Es ist nicht unmöglich, dass es eine Kontaminationsform von *ảzv- (in s. $a k k s ̧ u-)$ und *ỏrzu- (vgl. őr $\chi-v \eta)$ ist. Dann hätte man nicht nötig, es mit ỏzúc zusammenzustellen, was allerdings an und für sich wohl möglich wäre.

Dass der Name Ingvoeones (Ingcevones), ags. Ingwina (fréa) u. s. w. und das (durch Fick Ilias 376. 562 f.) dazu gestellte 'Axaıoi zu engh- in ÉrXoc gehören, ist von Noreen ausgesprochen worden (vgl. Verf. BB. XVIII 28).

\section{Skr. গ̊s̆ya-, rišya-.}

Beide Wörter sind gleichbedeutend. rišya- 'Bock der Gazelle' ist freilich nur Trik. 2,5, 6 bezeugt. Man könnte vermuten, sie seien formal identisch. Dagegen spricht, dass gr- und $r i$ - schwerlich identifiziert werden können, es sei denn, dass rišya- eine dialektische Entwickelung bezeichnen sollte. Mir ist wahrscheinlich, dass die beiden Wörter zu trennen sind.

'jucken', nëžai 'Krätze', vgl. lett. naifa 'Krätze' (s. Leslien Ablaut 18) und wahrscheinlich s. niksati, niksé 'durchbohren', wozu abg. nzza nzsti gezogen werden kann.

1) Es liegt nicht fern die Vermutung auszusprechen, dass -wic, lit. üsis und das dem d. Gesenke, slav. Jasenik, zugrunde liegende čech.-serb. jasen (Much Z. f. d. A. XXXIII 1 ff., (vgl. jetzt noch Meringer Beitr. z. Gesch. d. idg. Dekl. 8), abg. jasika 'Esche' u. s. w. mit dem dän.-norw.-schwed. (dial.) ōr, an. ōr, ōrir zusammenzustellen sind. Diese Wörter bedeuten dasselbe wie aisl. ǫlr, 1. alnus; die Bed. ist aber wahrscheinlich verschoben. Ein urger-

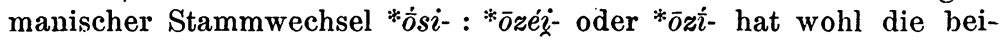
den unumgelauteten Formen ōr und ōrir ergeben können. 
rišya- ist idg. rik-îno-, ablautend mit roikko- in ahd. rēh, nhd. reh, ahd. rēho 'Reh', reia 'caprea', d. ricke aus *rizjo-. Dagegen fusst $r_{n}^{\prime} \dot{y} y a-$ auf einer Basis el-k-, l-k- in ahd. ëlch, ëlhe, ëlaho, agss. eolh, an. elgr, l. alces (bei Cäsar), ẳ $\kappa_{n}$ (Paus. V. 12, 1). Eine einfachere Wurzel el- ohne $k$ erscheint lit. elnis

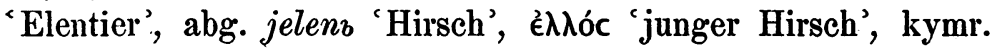
elain 'cerva'. Skr. èna., èñ̄ 'schwarze Antilope' können, wie dies Windisch KZ. XXVII 168 angenommen hat, hiermit nicht unmittelbar zusammengestellt werden. Entweder sind sie Lehnwörter aus einer europäischen Sprache, oder sie sind durch Vermischung von zwei Wörtern entstanden. Das n deutet auf eine Wortgruppe mit $l$ hin. Diese dürfte ind. *ana, *añ̄ gewesen sein, daneben kam aber auch eine andre Gruppe mit ähnlicher Bedeutung vor, nämlich (ēna-), éñ̄, ènt. Die Vermischung dieser Gruppen ergab ēnt und darnạch auch ēna-. Mag nun F. étñ̄, ènt durch Analogie entstanden sein (J. Schmidt Pluralb. 400) oder nicht, nichts hindert, dass es älter als diese Vermischung ist, somit ihr Grund sein konnte, wie es ja litterarisch fruher bezeugt ist. Was auch èñe, ènt und das dazu gehörende Mask. éta- ursprüunglich bedeutet haben - etwa ‘eilend, beweglich, schnell' (s. Grassmann s. v.) - sicher ist wohl die Bedeutung 'bunt, schimmernd, schillernd', vgl. êtaša-, ètašá'bunt glänzend, '(Sonnen)ross' und darnach 'Antilope' (vgl. Pischel Ved. St. I $225 \mathrm{f}$.), sei es dass diese Bedeutung aus der Beweglichkeit oder der Farbe herzuleiten ist. Übrigens ist lett. aita 'Schaf' (Fick BB. I 333) zu vergleichen.

\section{Skr. gühati 'verbergen'.}

Nach Fick I ${ }^{3} 315 .^{4} 195$ soll dies Verb eine Wurzel geugh- mit anlautender Media explosiva enthalten. Die av. Wörtel (vgl. z. B. av. gaozaiti, fraguzayañta, ap, gaudayāhy u. s. w.) können nicht entscheiden, ob $g$-oder $g h$ - anzusetzen ist. Von den sanskritischen scheinen jedoch z. B. aghukșat u. s. w. und noch einige nur bei Grammatikern belegten Formen (wie ghōkșyati, jughukșa- u. s. w.) gh- bezeugen. Ausführliche Besprechung des Vb. bei J. Schmidt KZ. XXV $164 \mathrm{ff}$. (vgl. Pluralb. 219 N. 1. Osthoff MU. IV 9).' Das Resultat ist

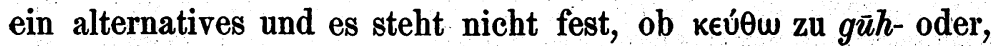
was vielleicht wahrscheinlicher, zu einer Wz. kudh- (oder idg. sqheudh- in g. skauda-raip, an. skaudiv 'Scheide', av. khao- 
dha- 'Helm') gehört (vgl. v. Bradke ZDMG. XL 667). Aus den germanischen Sprachen ist bis jetzt, soviel ich weiss, kein verwandtes Wort herangezogen. Ich werde hier eins vorschlagsweise vergleichen, das eventuell endgültig die Wurzelform ghŭgh- darthun wird. Das aisl. gȳgr 'ogress, witch' kann gar wohl seiner Bedeutung nach aus einer Wurzel 'verbergen, verhehlen' herzuleiten sein. Diese Anschauung. passt bekanntlich auf die Riesen und Riesinnen, die ja auclr Repräsentanten für Zauber und dergleichen geheimnissvolles Treiben sind ${ }^{1}$ ). Sie sind ja auch in den Felsen in der Erde verborgen. Den Versuch Brates BB. XIII $21 \mathrm{ff}$. gỹgr mit isl. $g \bar{o} i$, dem Monatsnamen, zu vereinigen hat Bugge Arkiv f. nord. fil. IV 123 endgriltig beseitigt.

35. Skr. náka- 'Himmelsgewölbe'.

Ich habe, soviel ich mich erinnern kann, keinen Versuch gesehen dies Wort etymologisch zu deuten. Hier ein Vorschlag. Bekanntlich ist die Bed. Himmel oft aus einer Bed. Gewölbe, und diese aus der allgemeinen Bed. krümmen, biegen entwickelt. Air. nem ist wohl weder mit Stokes BB. XI 96. 112 lautlich aus neb (= vé甲oc, s. nábhas) zu erklären, noch mit Brugmann Grundr. II § 132 S. 393 durch Anlehnung an

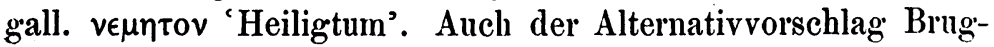
manns, dass es gleich s. námas sei mit dem ursprtinglichen Simne 'Gegenstand der Verehrung' scheint mir nicht ansprechend. Das s. námas, idg. némos, bedeutet urspr'unglich 'Biegung, Kriimmung; Gewölbe'. Daraus ist die Bedeutung. 'Himmel' entstanden. Mit diesem námas, air. nem, ist nun s. náka- wurzelverwandt. Ich deute es aus ${ }^{*} n \bar{n} q o-$, d. b. ${ }^{*} n \partial m q o-$, durch Reduktion einer zweisilbigen Wurzel neme-, nema- entstanden.

Dass wir es mit einer ursprünglichen Bedeutung 'Biegung.' $z u$ thun haben, wird bewiesen durch eine andre Entwickelung. 'So ist 1. nemus 'lucus' nichts als eine 'waldige, niedrige Vertiefung oder Senkung', wohl ursprïnglich etwa gleich Thal

1) Vgl. z. B. s. kuihaka- 'Gaukler', kuhara- 'Höhle', kǔhanā Heuchelei' der Wz. kudh- (idg. sqheudh-) 'verbergen'. Vgl. auch kuha-, als Beinamen des felsenbewohnenden Kuberas, 'des Vorstehers der Tiefe und des Dunkels, des Gottes der Schätze' (vgl. Fick I ${ }^{3} 50$. Hübschmann KZ. XXIV 412. Osthoff MU. IV 91. Bartholomae BB. X 290. A. F. I 176. v. Bradke ZDMG. XI 667). 
(vgl. Verf. P.-BrB. XIV 297 N. 1 f. $)^{1}$ ). Diese Bedeutung begegnet nun auch im Kelt. St. nantu- aus *nm-tu- 'Thal' (altgall. nanto Abl. 'valle', tri-nanto 'drei Thäler', Nantuates Plin. III 20, 24, Navtovátal Strabo IV 204, kymr. nant 'vallis', s. Glück 8. Zeuss-Ebel 764. 814. Stokes KSB. VI 229), wozu Part. s. natá- 'gebogen'. Hierzu mit starker Wurzelform gall. $\vee \in \mu \eta \tau o v$, nemeto-durum, air. nemed 'sacellum', eig. 'heiliger Hain' oder 'Hain' im allgemeinen (s. Glück $16 \mathrm{f}$. 75 . Zeuss-Ebel 10. 36. 40. 85. 87. 797. Stokes BB. XI 122 ff. 152) aus *neme-t(-o), wozu auch av. nemata-, nimata- 'Gras, Weide', altfrünk. nimid 'Weide', vgl. auch vémoc N. 'Weidetrift' $\wedge 480$. Die letzte Bedeutung ist wohl aus der Bedeutung ' $W$ ald, Thal' entwickelt.

Nachdem wir nun für das idg. némos die Bedeutungsentwickelung sowohl zu 'Thal, Niederung, Hain' (d. h. Gewölbe so zu sagen im Nadir) als zu 'Himmel' (d. h. Gewölbe im Zenith; vgl. altus 'hoch : tief') erkannt haben, können wir für das idg. nīqqo-, woraus s. náka- 'Himmelsgewölbe', und nmqo- auch die Bedeutung in entgegengesetzter Richtung erwarten. Ich stelle hierzu gr. vóm 'Waldthal, waldiger Thalgrund', aus

1) Die Entwickelung der Bed. 'Thal' aus 'biegen' ist bezeugt ausser durch die von Noreen (bei Hellquist Ark. VII 171) herrührende Identifizierung der beiden aisl. dalr 1. 'Bogen': 2. 'Thal' (wie auch 3. 'Hirsch' von dem gebogenen Geweih, Hellquist a. a. O.) auch durch die daselbst erwähnten Parallelen lett. leija 'Thal':

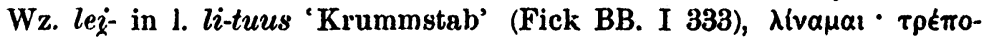

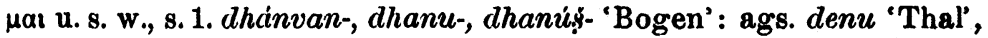
dene M. id. (St. Jani-Sievers P.-BrB. IX 243. Bugge Ark. VI 236, der jedoch eine andre Etymologie vorschlagt). Hiermit etymologisch zusammenhängend sind meiner Meinung' nach sowohl s. dhánu- 'Gestade' als 2. dhánvan- 'Festland, trockenes Land'. Die verschiedenen Bedeutungen lassen sich unschwer aus etwas 'bogenförmigem, sich erhebendem' herleiten. Zu lit. linkti 'sich biegen', lènkti 'beugen' gehört bekanntlich lankà 'Thal, Wiese'. Auf dieselbe Anschauung

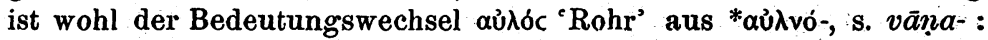
aủhúv 'Thal', l. vallis (s. Stokes MSL. V 421. Schrader KZ. XXX 472. Persson Wurzelerw. u. Wurzelvar. 230. Verf. GGA. 1890, 749. BB.XVIII13) zurückzufübren, ganz wie mhd. tülle 'Rohr' : d. Thal, an. dalr u. s. w. Man möchte die Frage aufwerfen, ob nicht möglicherweise dhānikā (in mandūra-dhānikā AV. XX 136, 10) 'weibliche Scham' bedeute (wie BR., Grassmann u. a. annehmen) und hierher zu stellen sei unter der Bedeutung 'Röhre'. Grundform*dhöl(a)-ni(*dhalnįé-). 
*nmøqa Fem., etwa r̂̂ váin. Mir scheint diese Deutung in formaler und begrifflicher Hinsicht den Vor\%ug vor den frilheren Erklärungen (z. B. von Fick $I^{3}$ 127) zu haben.

Hier noch ein parr Parallelen \%u dem Bedeutungswechsel 'Himmel(sgewölbe): Thal' in air. nem : l. nemus, vé

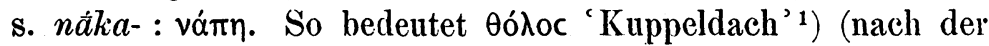
gewöhnlichen Auffassung ein Rundbau mit einem Kuppeldach $\chi$ 442. 459. 466), und das damit identische isl. dalr bedeutet 'Bogen', nach Hellquist Ark. VII 171 in Heimdale, Heimdall.' ( $n$-St. von dalr). Anderseits bedeutet ja dalv auch 'Thal', wie abg. dolz und s. dhära- 'Tiefe'. Hiermit wurzelverwandt sind wohl die schon genannten Wörter s. dhanús̆̌, dhánvan'Bogen' : ags. denu, F. dene M. 'Thal' ${ }^{2}$ ).

36. Skr. iyarti und Verwandtes.

Nach Grassmann Wb. s. v. $a r$, $\vartheta_{0}^{\circ}$ 'in Bewegung setzen' und Bartholomae A. F. II 69 ff. 76 ff. (vgl. jetzt auch Kretschmer KZ. XXXI 384) ist iyarti ein redupliziertes Präsens zu ar- in r’-nóti, ő $\rho-v u-\mu$ u. s. w. Nur bringt Bartholomae iyarti mit $\hat{\imath} r-t \bar{e}$, '́rate 'in Bewegung setzen' in der Weise zusammen, dass $\bar{\imath} r$ - eine analogice aufgekommene schwache Stammform des starken reduplizierten Stammes iyar- sei. Fick I ${ }^{4}$ 177. 365

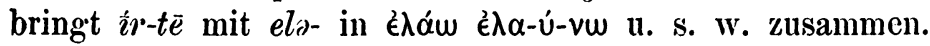

Grassmann-Bartholomaes Standpunkt ist mir am wenigsten annehmbar. Folgendes spricht dagegen. Zunächst

1) Ich erinnere hier an den sehr gewöhnlichen Wechsel 'Himmel': 'Dach'. D. himmel bedeutet 'caelun', aber ahd. himil hat auch die Bed. 'Dach', vgl. ndl. hemel 'Dach', ndl. gehemelte, schwed. siinghimmel 'Betthimmel'. So sucht Bartholomae BB. XV 197 in nákadie Bed. 'Dach' in RV. I 68, 10. 125, 5. IV 13, 5.

2) Falls man wagen dürfte die Wz. nem- 'biegen' in einfachere Elemente aufzulösen, könnte man daran denken, darin die einfache Wz. sen-, snē- 'biegen, winden, binden' in ahd. sënawa,

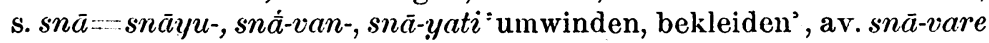
zu suchen; die erweiterte Wz. (s)n-em- verhielte sich dann zu sn-esin den von Lexikographen angeführten snas $\bar{a}$ Trik. 2, 16, 18. H. 631. Halāy. 3, 12, dalasnasà 'B 'Blattgefäss, Blattader', wie z. B. trem- (l.

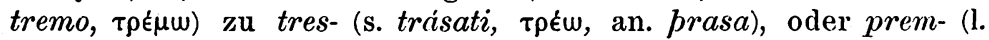
premo) : pres- (l. pres si, s. Danielsson Paulis Ait. St. IV 168 N. 1),

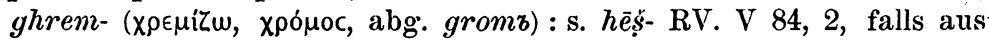
hrş- (v. Bradke ZDMG. XL 685), was jedoch sehr zweifelhaft ist (s. Pischel Ved. St. I 45 ff.), vielleicht bhrem- in s. bhramara-: bhres- in pr. bhasara Hem. I 244 (Pischel II 52). 
ist die Erklärung von $\bar{\imath} r$-, wie sie Bartholomae vorträgt, dass nämlich analogice nach einem ${ }^{*} i y$-aiti $:{ }^{*}{ }_{i}$-tai ${ }^{*} i y$-aišti $:{ }^{*} \bar{\imath}{ }^{z}$-tai u. s. w. zu iyarti ein ${ }^{*} \bar{r} r$-tai, s. $\bar{t} r$-te $\bar{e}$ gebildet worden sei, nicht allzu einleuchtend. Wahrscheinlicher wäre wohl $\bar{\imath} r$ - aus ${ }^{*} i$-ar-, d. h. s. *i-žr - (oder *io-tai, Brugmann IF. I 81) zu erklären. Auch ist die Kausativbildung ìráyati von diesem reduplizierten Stamm befremdlich, obwohl Analogien nicht ganz fehlen.

Übrigens wäre in begrifflicher Hinsicht die Ficksche Zusammenstellung mit idg. elo-, é $\lambda \alpha$ - weit ansprechender als Zusammenhang mit er- (or-), das hauptsächlich eine vertikale Bewegung zu bezeichnen scheint. Bei Annahme der Fickschen Anknüpfung hätte man ein idg. Paradigma *i(i)él-ti med. ${ }^{*} i(\underset{i}{i})$-al-tài anzusetzen, obwohl er selbst nicht eine solche Entwickelung anzunehmen scheint.

Jedoch scheint es mir in keinem sicheren Falle erwiesen, dass auf $a-, e^{-}, o^{-}$anlautende Wurzeln jemals mit Präsens. reduplikation $i(i)$ gebildet worden sind. Von den von Bartholomae A. F. II $69 \mathrm{ff}$. verzeichneten Fällen ist nur die sog.

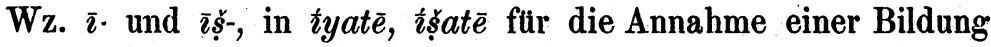
$i(i)-e i-($ ar. $i y-a y$ - in äiyēh) verwendbar. Indessen kann $i y-a y-$ :

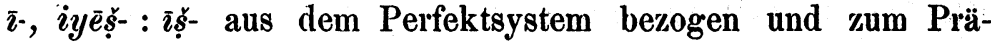
sensstamm umgebildet sein. S. $\bar{\imath} s-$ aus $a \dot{s}-$ herzuleiten verbieten besonders die verwandten Wörter aus den tibrigen idg.

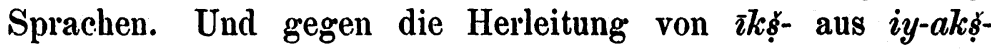
hat J. Schmidt Plurälb. 405 begrundeten Einspruch erhoben. Vgl. auch Pischel Ved. St. I 17.

Meiner Ansicht nach haben wir in s. mit $l$-Erweiterung versehene Form der Wz. ei - 'gehen'. Diese erweiterte Wz. war etwa (e)iel-, wozu ein redupliziertes Präsens *(i)i-iel-ti- gelautet haben mag. Schwache Wurzelform findet sich in *(i)i-il-tai, s. trr-tēe.

Hierzu stelle ich zunächst ahd. ìlen, illen (aus*īl-jan, hervorgegangen aus der schwachen Wurzelform des reduplizierten Präsens). Im Nomen idg. ${ }^{*} i l$-io- $=$ ags. $i l e$, aisl. $i l$ 'Fusssohle' (vgl. Kluge Wb. u. eilen).

Auch das griechische iád $\lambda \lambda \omega$ ist hierher zu stellen. Schon A. Kuhn KZ. V 193 ff. XIV 319 f. Curtius Et: ${ }^{5} 551$ baben dies Wort mit iyarti zusammengestellt ${ }^{1}$ ), unter der Annahme,

1) Dagegen Fick Spracheinh. 150. Wb. $\mathrm{I}^{3} 509 \mathrm{zu}$ s. işs- 'anreg'en', Aufrecht KZ. XIV $273 \mathrm{f}$. de Saussure Mém. $15 \mathrm{zu}$ s. sisarti. 
dass dies ein redupliziertes Präsens zu er-, or- sei. iád $\lambda \lambda \omega$ aus *i(i)- $i l-i \bar{o}$ ist gewissermassen ein Kausativum zum schwachen Stamm des ved. Präsens und stellt sich demnach am nächsten zu dem s. Kaus. ìráyati, womit es jedoch nicht völlig. identifiziert werden kann.

\section{Skr. ásita- 'dunkel'.}

Das Wort steht vielleicht für *msi-to- und bedeutet eigentlich 'befleckt', davon 'schwarz, dunkel'. Unter Annahme dieser Bedeutungsentwickelung' kann man es zu ahd. māsa' Wundmal, Fleck', mnd. mäse 'Fleck', ahd. mäsala 'Weberschlichte', mnd. massele 'Hautfleck, Ausschlag' u. s. w. ziehen. Man hätte als 'Wurzel' etwa mĕe-s- : mə-s- : m-s- anzunehmen. Vielleicht gehört hierher auch ắcl-c 'Schlamm, Unrath' bei Hom. $\Phi 321$, das verschieden erklärt worden ist (am besten von Froehde BB. VII $85 \mathrm{zu}$ l. sent-īna 'Bodensat\%'). Grundform ${ }^{*} m s-s-i$-, ausgegangen von einem $s$-St. *mĕ-s-os- Gen. ${ }^{*} m-s-s-e ́ s$.

Die Heranziehung von ăcıc dürfte nicht allzu gewagt scin, wenn man bedenkt, dass der Flussname Ásiknī oder Asiknt̀ ('Aкєcínc) vielleicht eben 'die schlammige' bedeutet. Über die Fem.-Bildung ási-k-ñ̄ s. J. Schmidt Pluralb. 398.

\section{Skr. ártnī 'Bogenende'.}

Das Wort bedeutet 'das Bogenende, wo die Sehne befestigt wird' und wird allgemein von $\bar{a}$ und $a r$ - 'hinzufügen' hergeleitet. Wahrscheinlicher scheint mir, dass es nur eine Schwesterform zu árat-ni- 'Elnbogen, Elle, Winkel, Ecke' sei. Formell ist es aus * $\delta l-t-n \bar{\imath}$ (aus *ole-t-n-) $\%$ deuten. Es verhält sich ártn̄ zu áratni- wie s. äni- 'Zapfen der Achse, der in der Nabe des Rades läuft' (aus *ǒl-ni-) zu aráni-, aranin, ahd.

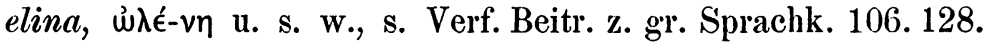
141 f. Hierzu lett. ula 'Radnabe', av. rāna 'Schenkel' (vgl. Bezzenberger BB. XVII 215). Eine Kombination des $n$ - und $s$-Stammes (im ags. alor, abg. jelb-ch-a u. s. w.) etwa wie s.

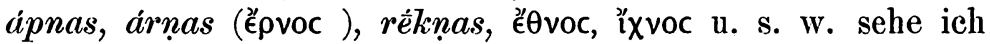
in lünse, mhd. luns(e), as. lunisa, ags. lynes, g. *lunizi (s. Kluge $\mathrm{Wb}^{4}$ u. lünse) aus *la-ne-s- (*(ŏ)lé-no-s- : $\left.{ }^{*} l a-n e ́-s\right)$. As. lunisa u. s. w. verhält sich zum no-s-St. etwa wie g. ga-, ur-runs, ahd. runs zu s. ár-na-s, arnas-á-und, abgesehen von den Wurzelsilben, verhält sich lunisa zu s. äní-, aṇi- (vgl. Fick 
13B. VII 95. I ${ }^{4} 123$ ) wie g. -runs, s. árnas zu g. (Dat.) runa, ags. ryne, s. árna- (vgl. Persson Wurzelerw. u. Wurzelvar. 102 N. 2). Auch möchte ich s. ánu- 'fein, dünn' (aus *ol-n-u-) hierher ziehen, indem ich von der Bedeutung 'biegsam' ausgehe (anders einerseits Bury BB. VII 342, anderseits Fortunatov BB. VI 216. Hübschmann ZDMG. XXXVIII 428. E. Kuhn KZ. XXX 355).

Ich führe nämlich alle diese Wörter auf die Basis ŏlě'biegsam, beweglich sein', die auch in lit. ülektis, ő̉ $(\alpha) \xi$, å̀ $\alpha$ кóta u. s. w. (s. Verf. a. 0.) erscheint. Diese Basis erscheint ohne besondere Weiterbildungen (ich sehe naturlich vom thematischen Vokal ab) in s. ar-ala- 'gebogen, gebogener Arm', wo ich andern (z. B. Curtius Et. ${ }^{5}$ 374) gegenüber ar- als Präfix und -äla- als Nom.-Verbalstamm, nicht als Suffix, fasse. Besonders ist das folgende Wort hervorzuheben. S. viala AV. V 22, 6 wird verschieden ubersetzt. Ludwig Rigv. III 510 deutet es als 'Schlange'. Grill Hundert Lieder d. Ath.-Veda 12 übersetzt es mit 'tückisch' in Übereinstimmung mit den meisten Auslegern. Beide Bedeutungen sind in der klassischen Sprache geläufig. Dass dies eine Zusammenset/ung aus vi-

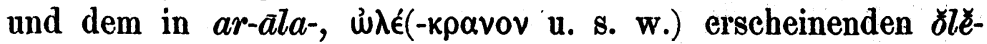
sei, ist höchst wahrscheinlich. Nur fragt es sich, unter welcher Entwicklungsformel die Bedeutungen 'Schlange' und 'tuckisch' vereinigt werden sollen. Man kann von einer Grundbedeutung 'in verschiedenen Seiten gebogen' $z u$ 'windig, unverlässlich, boshaft, tulckisch' und weiter zu 'Schlange' kommen. Man kann aber zur letzten Bedeutung auch auf anderm Weg gelangen. Wir können in ala eine substantivische Bedeutung etwa 'Glied' und in $v i$ - eine Privativ part. sehen und víala- als 'ohne Glieder' deuten. So heisst die Schlange in derselben Stelle vianga-, das sicher sowohl dort als AV. VII 56, 4 doch nur 'gliedlos' bedeuten kann. Auf derselben .Anschauung beruhen andre Benennungen der Schlange: urága(aus *ur-n-go-) 'Brustgänger, Schlange' (vgl. auch ura-m-ga-, -gama- 'Schlange'), wie alle die Namen, die auf 'winden, drehen' bezug haben wie kromi- (lit. kìmis, kirmèlë, air. cruim, kymr. pryf) \%u qer- 'krümmen', s. kīta- 'Wurm' zu qel'winden, krümmen'. Aus andern Sprachen 1. vermis, fó $\mu \circ c$,

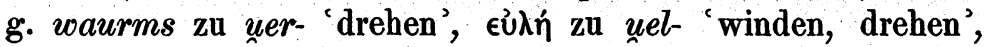
é $\lambda \mu \mathrm{ic}$ entweder zu uel-oder zu süel- u. s. w. 
Noch eine Wortgruppe möchte ich in Beziehung zu dieser Sippe bringen, nämlich așsthî-vánt- 'Knie(scheibe)' Ait. Br. II 6, 15 (vgl. Komm. Çān̄kh. Çr. S. 4, 14, 32: așthīvatšabdēna šuș̌kasañghē ucyētēe), aș̣thīla 'Kugel, runder Stein, Kiesel, Ambos, Obstkern, kugelige, steinharte Anschwellung im Unterleibe', wozu aștthīlika 'eine Art von Eitergeschwüren', ürv-așth $\bar{\imath} v a$ - N. 'Kniescheibe'. Ich sehe in așthī- ein ursprüng-

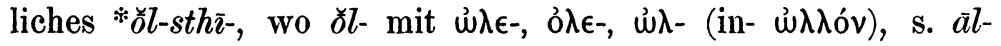
(in viāla-, arāla-) identisch ist; -s-thi- könnte ein Suffix sein dessen $s$ - eigentlich mit ŏ $l$ - zu einem $s$-St. ŏl(e)-s- gehörte. Wahrscheinlicher ist mir aber, dass -sthī- schwache Form von idg. *osthi- 'Knochen' ist: *ǒl-stȟ̆- heisst somit 'gebogener Knochen' *ǒl-stȟ̌-uent- 'mit gebogenem Knochen versehen', d. h. 'Knie(scheibe)'. Aus dieser Grundbedeutung erklären sich alle unter așththla verzeichneten Bedeutungen vorzüglich.

Von andern Wörtern, die vermutungsweise hierher gezogen werden können, verzeichne ich äla- 'name af a creeper or weed in grainfields' Käuç. S. 25, 18, silán̂́j-āla- 'a creeper or weed growing in grainfields' AV. VI 16, 4. Kāuç. S. ō1, 16; alas-āla-, nīlāgalas-ālā AV. VI 16, 4, asit-āla- Kāuç. S. 35, 28 harit-äla (s. BR. VII 1551. Bloomfield Einl. zu Kāuç. S. JAOS. XIV, XLV), vielleicht auch aläk $\ddot{a}$ 'name of a plant' Käuç. S. 31. 28.

39. Skr. usrá- und Verwandtes.

S. usr $a^{-}$- ist von Frochde BB. II 337. J. Schmidt Pluralb. 201 mit an. ür Gen. urar zusammengestellt. Völlige Gleichsetzung durfte jedoch lautgesetzlich nicht möglich sein, obwohl noch neulich Kluge $\mathrm{Wb} .^{5}$ dies angenommen hat. Zur Not könnte man für $\bar{u} \cdots$ ein idg. ūsró- annehmen, woraus möglicherweise *üzrás - *uzraz - *üRraR - ūr (Noreen Urg. judl. 112). Aber teils ist diese Entwickelung Zweifeln unterworfen, teils sind die Grundformen nicht identisch.

Man hat allgemein usrá- 'Stier', usráa 'Kuh' mit usrá'rötlich, glänzend, morgendlich' identifiziert. Dies ist jedoch nichts weniger als sicher. Ich vermute eine ganz andre Etymologie.

Zunächst ist usrá- mit riștra- 'Büffel' und ușttár- 'Pflugstier" zusammenzuhalten. Diese Bildungen aber sind Nom. agentis, was gar nicht mit der Herleitung aus einer Bed. 'röt- 
lich'stimmt. Vielmehr werden wir dadurch auf ganz andre Fährte geleitet.

Sowohl vŕş̧an-, vrş̧abhá-, el. Fó $\rho \rho \eta v$ (SGD. 1152, vgl. Fick

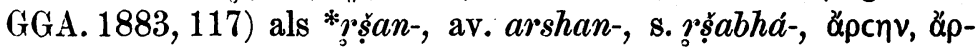

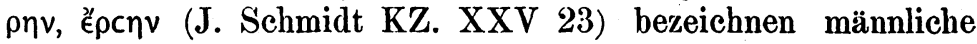
Tiere und sind deutlich von der Bed. 'netzen, semine irrigare' der Wurzeln uers- und ers- ${ }^{-}$) benannt. Zu uers- noch s. vorş̌ni'Widder' 2), lit. vèrszis 'Kalb', l. verrēs. Ja, s. vrș̣a- bedeutet eben 'taurus' (vgl. vrsşana- 'testiculus'). Überhaupt scheinen männliche Tiere sehr oft mit Wörtern, die auf 'netzen, Wasser' u. s. w. bezug haben, benannt gewesen zu sein (vgl. z. B. noch s. ukṣáan- 'Stier' : ukșáti 'netzen, träufeln'. Und es ist mir sehr wahrscheinlich, dass dies auch in usrá- u. s. w. der Fall gewesen ist.

Die Wz. uer-s- ist deutlich eine nominal-verbale $s$-Erweiterung des St. uěrr- in s. várr(-i) 'Wasser', an. ür 'feiner Regen', 1. ürīna, lit. júrès Pl. 'Meer', pr. jürin (vgl. Bugge KZ. XX 29. J. Schmidt Pluralb. 204), wozu vielleicht noch urva-, urvi- (oder urvya-) in av. urvapa-, urvyāpa-, worin wohl tautologische Zusammensetzungen enthalten sind ( $v g l$.

1) Als Nom.-Stämme in s. varş่á-, Éépcn, l. rōs (aus *u(e)rós) : s. rása, rasā, abg. rosa, lit. rasà, u. s. w. Im Vorübergehn einige Worte über die $s$-Erweiterung der Wz. er-, or-. Es laufen neben einander Stämme er-u-, er-n-, er-s-, er-i- u. s. w.: av. aur-v-a, s. $a r-$

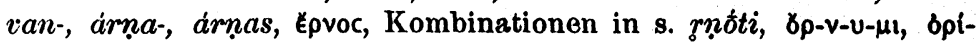

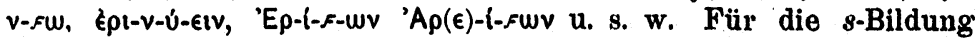
haben wir eine Basis érðs-, éros-, orés-, arés- vorauszusetzen, wie auch ers-, Ørs-, rěs-, rðss. Die Form erðs- ist in Epwn 'heftige Bewegung, Schwung' (Kombinationsform aus den beiden Fem.-Bildungen *erós und *ersă wie 1. aurōra u. s. w. Verf. GGA. 1890772 ff.) vorhanden, erəs- in 'éćw 'ausgiessen, auswerfen (Fick KZ. XXI 375, vgl. Froehde BB. VII 119). Die Form ərés-sehe ich iras-yati 'zür-

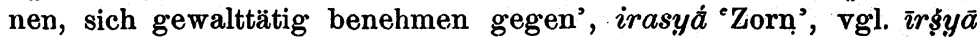
'Neid', ags. yrsian 'irasci' (Froehde BB. V 270. J. Schmidt Pluralb. 364). Eine Flexion eros gen. arés-os oder (a)r(o)s-és konnte leicht einen Stamm mit doppeltem $s$ exzeugen (o)res-os- (vgl. Verf. KZ. XXX 422). Diesen Stamm erkenne ich in abg. rosa aus *os-s-ä, dann vielleicht auch in lit. rasà und möglicherweise s. rása, obwohl dies auch eine 'Weiterbildung' des einfachen s-Stammes (o)ressein kann.

2) Als Adj. 'stark, kräftig'; über das zugehörige Neutr. vว̊ṣ̆ni s. J. Schmidt Pluralb. 247. 
Darmesteter Et. iran. II 180. Geldner KZ. XXVIII 187 N. 2), vielleicht auch noch s. var-ti- 'Urethra' Kāuç. S. 25, 16.

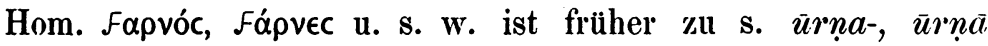
'Wolle' u. s. w. gestellt, was natürlich nicht angeht (Wz. uel-). Auch die neueste Herleitung Fick $\mathrm{I}^{4} 550$, F $\rho \eta v$ - gleich ${ }^{*} f \rho \in \mathrm{c} v-$ zu setzen u. s. w., ist unbefriedigend. Am besten scheint man hier von der Basis ǔĕ-r- auszugehen und die urspr. Bedeutung. 'Benetzer' als Benennung des männlichen Tieres anzunehmen.

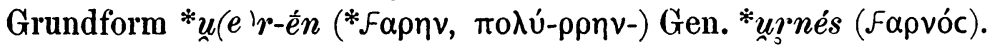
Die griech. Bedeutung ist freilich 'Schaf' ( $\pi \rho$ ó $\beta \alpha \tau o v)$ im allgemeinen, speziell 'Lamm', Indessen ist doch die Bedeutung. als männliches Tier noch vorbanden in $\alpha$ $\rho \imath-\chi \alpha \cdot \alpha ُ \rho \rho \in \nu \pi \rho o ́ \beta \alpha-$

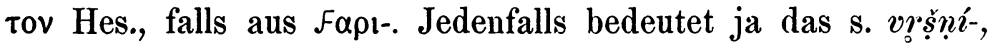
av. varshni-, von der $s$-Form gebildet, 'Widder'. Ob s. urana‘aries' idg. *ur-eno- (und hierher zu stellen) oder *ul-eno- (zu uel-) ist, lässt sich nicht entscheiden.

$\mathrm{Zu}$ der $r$-Form ${ }^{1}$ ) der Basis ziehe ich nun an. ür, ahd., mhd., ags. ur, urgerm. *ür-os, woraus wohl lat. ürus entlehnt ist. Das deutsche $\bar{u} r$ sowohl in $\bar{u} r$ (-ohso) als in $\bar{u} r$-han lässt sich als ein und dasselbe Wort erklären, eben unter Annahme der Bed. 'männliches Tier'.

Kann freilich auch nicht germ. *üraz mit s. usrá-identifiziert werden, so dürfen sie doch als verwandt gelten. Es ist wohlbezeugte Thatsache, dass $r-, s$ - und $n$-St. mit einander wech-

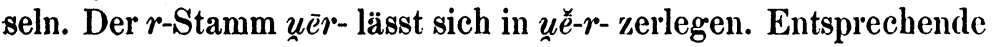
8- und $n$-Stämme mit derselben Bedeutung lassen sich auch nachweisen (s. Persson Wurzelerweiterung u. Wurzelvariation 47) $\left.{ }^{2}\right): n$-St. in s. aváni- 'Strom, Flussbett' (unsicher), vá-na-

1) Den $r$-Stamm noch in lett. varavikksne 'Regenbogen', lit. oráryksztè, vaivoriksztis (Geitler Lit. Stud. 119) s. Bezzenberger Lett. Dial.-St. 77 N. 2 f.

2) Die. Wz. eque- 'nass sein' tritt unter mehreren Formen auf, s. Verf. Beitr. z. gr. Sprachk. $117 \mathrm{f} .130 \mathrm{f}$. $149 \mathrm{ff}$. 154 . Persson Wurzelerw. u. Wurzelvar. 47. 85. 98. 228. 284. Hier noch zwei Belege: lit. audra 'Gewässer, Überschwemmung' (Bezzenberger ZGLS.

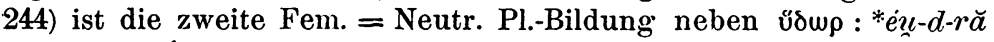
gen. *u-d-rás; av. ao-ta- 'kalt' dürfte eig. wohl 'nass' bedeutet haben, vgl. das dazu von Neisser BB. XVII $244 \mathrm{ff}$. gestellte ved. òmán- in der Bed. 'Kälte'. - Ich habe Beitr. z. gr. Sprachk. $150 \mathrm{~s}$ $a v a-t a ́$ - 'Brunnen' zur Wz. eque- 'nass sein' gestellt und nur als möglich angenommen, dass es idg. *euoto- sei; für lett, avuits habe ich zwei 
'Wasser" und nach Hellquist der an. Seename Vönir mit Wur-

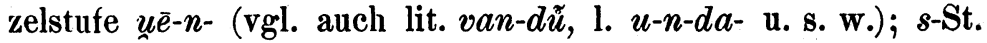
in ags. woces 'Wasser', ahd. wasulun 'pluviis' und mehrere Wörter mit Wurzelstufe us-, die Hellquist Ark. f. nord. fil. VII 42 gedeutet hat; möglicherweise auch ahd. waso 'Rasen, feuchter Erdgrund', wasal 'feuchte Erdmasse', wofur jedoch eine andre Erklärung von Noreen Urg.judl. 130 gegeben ist. Vielleicht ist von der neueren Wurzelform ein $s$-St. in dem von Geldner KZ. XXVIII $186 \mathrm{f}$. nachgewiesenen av. avō 'Wasser'. gebildet, das er gleich *abhas (d. h. ${ }^{*}$ mbhos) $=$ s. ámbhas setzt, das aber ein idg. *euos sein kann. Jedenfalls steht die Basis $u_{-}-s^{-}$'Wasser, nass sein, benetzen' ausser Zweifel. Hierzu ziehe ich nun s. us-rá-, uṣ́-tár-, uș̆-tra- 'Stier' (av. ushtra'Kamel'), d. h. eig. 'der Benetzer', wie vrişan-, ürr u. s. w.

\section{Skr. at- 'gehen'.}

Ich beanspruche nicht eine evidente Etymologie dieser Wortsippe zu geben; folgendes spreche ich nur vermutungsweise aus. Die Wz. at- kommt $5 \mathrm{mal}$ in $\mathrm{RV}$. vor sám-atasi I 30, 4, átann (súryậ) IV 61, 9, átamãnam II 38, 3, -ặ IV 9, 2 mit klarer Bed. 'wandern, gehen'. So hat man dieselbe W\%. auch in einigen andern Wörtern gesehen: viáti- 'Ross', atya- id., atasi- 'Bettler', atithin- 'wandernd', wohl auch atithi- 'Gast' ') (= av. astish Bartholomae BB. XV 10 f.). Nimmt man als Wechselbedeutungen 'gehen' und 'gehen lassen, entsenden' so kann man at- aus mot- erklären und zu lit. metì 'werfe', abg. meta 'werfe', die nicht mit 1. mitto identifiziert werden

Möglichkeiten erwähnt: *eūoto- oder *eu-ant- (*eu-ont-). Jetzt ist es mir wahrscheinlicher, dass s. avatá- aus *eu-n-t-ó- entstanden ist, wir also eine thematische Erweiterung des in an. unnr, ahd. Winida u.s.w. (s. a. a. 0.118 .130 f.) erscheinenden $t$-St. vor uns haben. Dies scheint mir geraten sowohl wegen lett. avrits, das doch am besten aus *euont(o)- hergeleitet wird, als auch wegen der Bildung s. avatká- AV. II 3, 1, die man doch lieber aus einem St. avat- (d. h. *avnt-) als aus einem avata- deutet,in welchem Falle man *avataka (was freilich eine - unmetrische - Lesart der Pāipp.-Rezension ist) erwartet, vgl. eine Bildung wie āmìvat-ká- 'andringend, drängend' aus *-mīu-nt-qo-, e ejatkä-. Über avatká- vgl. übrigens Grill Hundert Lieder d. Ath.-Veda $79 \mathrm{f}$.

1) Vgl. zur Bedeutungsentwicklung s. abhyatati, das nach den ind. Lexikogr. eben 'besuchen, einkehren' bedeuten soll. 
können, stellen. Diese Bedeutung kehrt auch im Skr. wieder, nämlich in atasá-, das ausser 'Wind, Seele' auch 'Geschoss' bedeutet. Ob das rigvedische atasá- N. 'Gebüsch, Gestrüpp' zur Wz. at- gehört, ist freilich unsicher; unmöglich ist es nicht unter Annahme einer Grundbedeutung. 'Sammlung von Schösslingen'.

Dass die Wz. at- 'wandern' atati, -tē u. s. w. eine jüngere Form von at- sei (Whitney Wurzeln 2. Pischel ZDMG. XXXV 722 f.), ist nicht sicher. Man kann at- aus al-t- deuten

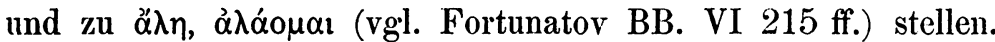

Die meisten der vorstehenden Etymologien sind allmählich seit 1887, namentlich im Sommer 1888, entstanden. Von den damals niedergeschriebenen Artikeln sind mehrere nachher ausgeschieden oder geändert worden, weil ihre Resultate inzwischen vorweggenommen "worden sind; einige von diesen sind jedoch stehn geblieben, einerseits weil sie teilweise anders gefasst sind, andrerseits weil sie noch einige Nebenfiragen behandelten, die möglicherweise verdienen berücksichtigt zu werden. Einige neue Etymologien sind an Stelle der ausgeschiedenen hinzugekommen. Diese stammen grösstenteils aus dem Sommer 1890. Verschiedene wichtigern Nachweise aus der letzten sprachwissenschaftlichen Litteratur sind vor der Absendung des Manuskripts noch nachgetragen worden.

1) Übrigens kann auch met- in $\mu \in \tau \alpha$, g. mip u. s. w. mit metin abg. meta identisch sein. Man bedenke nur, dass $\mu \in \tau \dot{\alpha}$ mit $\pi \epsilon \delta \alpha$ gleichbedeutend ist, und dies gehört ja doch zur W\%. perl-, s. pad'gehen' (über $\pi \epsilon \delta \&$ vgl. Osthoff Perf. 574. Bugge P.-BrB. XII 420. Schulze Quaest. hom. 54; Belege bei Meister I 117. 284. II 118).

Berlin, im September 1891.

Karl Ferdinand Johansson. 